\title{
Convergence of Perturbation Expansions in Fermionic Models. Part 2: Overlapping Loops
}

\author{
Joel Feldman ${ }^{1, \star}$, Horst Knörrer ${ }^{2}$, Eugene Trubowitz ${ }^{2}$ \\ 1 Department of Mathematics, University of British Columbia, Vancouver, B.C., Canada V6T 1Z2. \\ E-mail: feldman@math.ubc.ca \\ 2 Mathematik, ETH-Zentrum, 8092 Zürich, Switzerland. \\ E-mail: knoerrer@math.ethz.ch; trub@math.ethz.ch
}

Received: 21 September 2002 / Accepted: 12 August 2003

Published online: 6 April 2004 - (c) Springer-Verlag 2004

\begin{abstract}
We improve on the abstract estimate obtained in Part 1 by assuming that there are constraints imposed by 'overlapping momentum loops'. These constraints are active in a two dimensional, weakly coupled fermion gas with a strictly convex Fermi curve. The improved estimate is used in another paper to control everything but the sum of all ladder contributions to the thermodynamic Green's functions.
\end{abstract}

\section{Contents}

V. Introduction to Part $2 \ldots \ldots \ldots \ldots$. . . . . . . . . . . . 244

VI. Overlapping Loops . . . . . . . . . . . . . . . . . . . . . . . . . . . . . . . . . . . . . . . . . . . . . . . 244

VI.1 Norms . . . . . . . . . . . . . . . . . . . . 244

VI.2 Ladders . . . . . . . . . . . . . . . . . . . . . 247

VI.3 Overlapping Loops for the Schwinger Functional . . . . . . . . . 250

VI.4 Configurations of Norms with Improved Power Counting . . . . . 252

VII. Finding Overlapping Loops . . . . . . . . . . . . . . . . . . . . 256

VII.1 Overlapping Loops Created by the Operator $\mathcal{R}_{K, C} \ldots \ldots$. . . . . 256

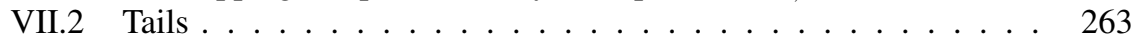

VIII. The Enlarged Algebra . . . . . . . . . . . . . . . . . . . . . 272

VIII.1 Definition of the Enlarged Algebra . . . . . . . . . . . . . . . . 274

VIII.2 Norm Estimates for the Enlarged Algebra . . . . . . . . . . . . 275

VIII.3 Schwinger Functionals over the Extended Algebra . . . . . . . . 276

VIII.4 A Second Proof of Theorem IV.1 . . . . . . . . . . . . . . . . . . 278

VIII.5 The Operator $Q \ldots \ldots$. . . . . . . . . . . . . . . 280

IX. Overlapping Loops Created by the Second Covariance . . . . . . . . . . 282

IX.1 Implementing Overlapping Loops . . . . . . . . . . . . . . . . . 283

^ Research supported in part by the Natural Sciences and Engineering Research Council of Canada and the Forschungsinstitut für Mathematik, ETH Zürich. 
IX.2 Tails ......................... 290

IX.3 Proof of Theorem VI.10 in the General Case . . . . . . . . . . . 298

X. Example: A Vector Model . . . . . . . . . . . . . . . . . . . . 307

Appendix C. Ladders Expressed in Terms of Kernels . . . . . . . . . . . . . . 314

Notation . . . . . . . . . . . . . . . . . . . . 318

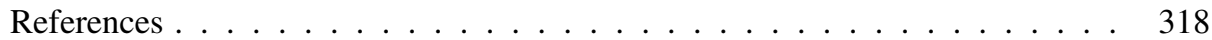

\section{Introduction to Part 2}

In the perturbative analysis of many fermion systems with weak short-range interaction in two or more space dimensions, the presence of an overlapping loop in a Feynman diagram introduces a volume effect in momentum space that leads to an improvement to "naive power counting". For a detailed discussion of this effect and its consequences, see [FST1, FST2, FST3, FST4]. For a short description, see Subsect. 4 of [FKTf1, §II]. In [FKTo3], we use nonperturbative bounds for systems, in two space dimensions, that are based on the cancellation scheme between diagrams developed in Part 1 of this paper. In this second part, we modify the construction so that we can exploit enough overlapping loops to get improved power counting for the two point function and the non-ladder part of the four point function. As in Part 1, the treatment is in an abstract setting, formulated using systems of seminorms. The postulated volume improvement effects are expressed in terms of these seminorms (Def.VI.1). The main result for the renormalization group map is Theorem VI.6. It follows from Theorem VI.10, which is the main result on the Schwinger functional.

The discussion of the renormalization group map in the first part of the paper is based on the representation developed in [FKT1] (which in turn evolved out of the representation developed in [FMRT]). The representation of [FKT1] decomposes Feynman diagrams into annuli. The first annulus consists of all interaction vertices directly connected to the external vertices. The second annulus consists of all interaction vertices directly connected to the first annulus but not to the external vertices. And so on. See the introduction to [FKT1]. Overlapping loops that only involve vertices of neighbouring annuli are relatively easy to exploit. It turns out, that for the analysis of the two point function and the non-ladder part of the four point function, it suffices to use overlapping loops that involve only vertices of at most three adjacent annuli. A special case of Theorem VI.10, for which this combinatorial fact is easier to see, is proven at the end of $\S$ VII. After some preparation in $\S$ VIII, the general case is proven at the end of $§ I X$. In $\S \mathrm{X}$, we apply Theorem VI.6 to a simple vector model. We also describe, by drawing an analogy with the vector model, how sectors can be used to nonperturbatively implement overlapping loops for many fermion systems. A notation table is provided at the end of the paper.

\section{Overlapping Loops}

VI.1. Norms. Again, let $A$ be a graded superalgebra and $A^{\prime}=\bigwedge_{A} V^{\prime}$ the Grassmann algebra in the variable $\psi$ over $A$. Also fix two covariances $C$ and $D$ on $V$.

Definition VI.1. Let $\|\cdot\|$ and $\|\cdot\|_{\mathrm{impr}}$ be two families of symmetric seminorms on the spaces $A_{m} \otimes V^{\otimes n}$ such that $\|\cdot\|_{\mathrm{impr}} \leq\|\cdot\|$ and $\|f\|_{\mathrm{impr}}=0$ if $f \in A_{m} \otimes V^{\otimes n}$ with $m \geq 1$. We say that $(C, D)$ have improved integration constants $\mathfrak{c} \in \mathcal{N}_{d}, \mathrm{~b}, J \in \mathbb{R}_{+}$for the families $\|\cdot\|$ and $\|\cdot\|_{\text {impr }}$ of seminorms if $\mathrm{c}$ is a contraction bound for the covariance 
$C$ for both seminorms $\|\cdot\|$ and $\|\cdot\|_{\mathrm{impr}}, \mathrm{b}$ is an integral bound for $C$ and $D$ for both seminorms and the following triple contraction estimate holds:

Let $n, n^{\prime} \geq 3 ; 1 \leq i_{1}, i_{2}, i_{3} \leq n$ and $1 \leq j_{1}, j_{2}, j_{3} \leq n^{\prime}$ with $i_{1}, i_{2}, i_{3}$ all different and $j_{1}, j_{2}, j_{3}$ all different. Also let the covariances $C_{1}, C_{2}, C_{3}$ each be either $C$ or $D$ with at least one of these covariances equal to $C$. Then for $f \in A_{0} \otimes V^{\otimes n}, f^{\prime} \in A_{0} \otimes V^{\otimes n^{\prime}}$,

$$
\left\|\underset{i_{1} \rightarrow j_{1}}{\mathcal{C o n}_{C_{1}}}{\underset{i}{i_{2} \rightarrow j_{2}}}_{\mathcal{C o n}_{C_{2}}} \underset{i_{3} \rightarrow j_{3}}{\mathcal{C o n}_{C_{3}}}\left(f \otimes f^{\prime}\right)\right\|_{\text {impr }} \leq J \mathrm{~b}^{4} \mathfrak{c}\|f\|\left\|f^{\prime}\right\| .
$$

Observe that $\mathcal{C o n}_{C_{1}} \quad \mathcal{C o n}_{C_{2}} \quad \mathcal{C o n}_{C_{3}}\left(f \otimes f^{\prime}\right) \in A_{0} \otimes V^{\otimes\left(n+n^{\prime}-6\right)}$.

$$
i_{1} \rightarrow j_{1} \quad i_{2} \rightarrow j_{2} \quad i_{3} \rightarrow j_{3}
$$

Lemma VI.2. Assume that $(C, D)$ have improved integration constants $\mathfrak{c}, \mathrm{b}, J$ for the families $\|\cdot\|$ and $\|\cdot\|_{\text {impr }}$ of seminorms. Let $n_{1}, \cdots, n_{r}, n_{r+1}, \cdots, n_{r+s} \geq 0$, let

$$
\begin{aligned}
& f_{1}\left(\xi^{(1)}, \cdots, \xi^{(r)}\right) \in A_{0}\left[n_{1}, \cdots, n_{r}\right], \\
& f_{2}\left(\xi^{(r+1)}, \cdots, \xi^{(r+s)}\right) \in A_{0}\left[n_{r+1}, \cdots, n_{r+s}\right],
\end{aligned}
$$

and let $i_{1}, i_{2}, i_{3} \in\{1, \cdots, r\}$ and $j_{1}, j_{2}, j_{3} \in\{r+1, \cdots, r+s\}$. Also let the covariances $C_{1}, C_{2}, C_{3}$ each be either $C$ or $D$ with at least one of these covariances equal to C. Then

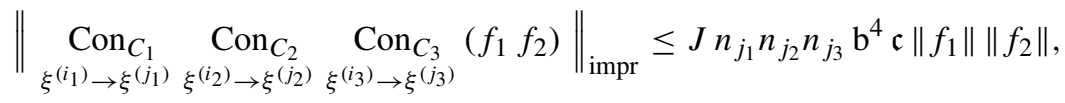

$$
\begin{aligned}
& \xi^{(1)}, \cdots, \xi^{(r)} \overbrace{f_{1}}^{C_{1}} \overbrace{C_{2}}^{C_{2}} f_{f^{(r+1)}, \cdots, \xi^{(s)}}
\end{aligned}
$$

Proof. The proof is analogous to that of Lemma II.29.i.

We define, for a Grassmann function $f$ the improved norm $N_{\text {impr }}(f)$ as in Def. II.23. That is,

$$
N_{\text {impr }}(f ; \alpha)=\frac{\mathfrak{c}}{\mathrm{b}^{2}} \sum_{n_{1}, \cdots n_{r} \geq 0} \alpha^{|n|} \mathrm{b}^{|n|}\left\|f_{0 ; n_{1}, \cdots n_{r}}\right\|_{\text {impr }}
$$

An abstract example of such norms is described at the end of this section, and this abstract example is made concrete in $\S \mathrm{X}$.

Definition VI.3. Let $f\left(\xi^{(1)}, \cdots, \xi^{(r)}\right)$ be a Grassmann function and $I \subset\{1, \cdots, r\}$. We say that $f$ has degree $d$ in the variables $\xi^{(i)}, i \in I$ if

$$
f \in \bigoplus_{\substack{m ; n_{1}, \cdots, n_{r} \\ \sum_{i \in I_{i}=d}=n_{m}}} A_{m}\left[n_{1}, \cdots, n_{r}\right],
$$

where $A_{m}\left[n_{1}, \cdots, n_{r}\right]$ was defined in Def. II.21. We say that $f$ has degree at least $d$ in the variables $\xi^{(i)}, i \in I$ if $f=\sum_{d^{\prime} \geq d} f_{d^{\prime}}$, where each $f_{d^{\prime}}$ has degree $d^{\prime}$ in the variables $\xi^{(i)}, i \in I$. Similarly we say that $f$ has degree at most $d$ in the variables $\xi^{(i)}, i \in I$ if $f=\sum_{d^{\prime} \leq d} f_{d^{\prime}}$, where each $f_{d^{\prime}}$ has degree $d^{\prime}$ in the variables $\xi^{(i)}, i \in I$. 
Proposition VI.4. Let $(C, D)$ have improved integration constants $\mathfrak{c}, \mathrm{b}, J$. Let $r \geq t \geq$ $s \geq 1$, and let $f_{1}\left(\xi^{(1)}, \cdots, \xi^{(s)}, \xi^{(s+1)}, \cdots, \xi^{(t)}, \xi^{(t+1)}, \cdots, \xi^{(r)}\right)$ and $f_{2}\left(\xi^{(1)}, \cdots\right.$, $\left.\xi^{(r)}\right)$ be Grassmann functions. Set

$$
\begin{aligned}
& g\left(\xi^{(t+1)}, \cdots, \xi^{(r)}\right) \\
& =\iint:: f_{1}\left(\xi^{(1)}, \cdots, \xi^{(s)}, \cdots, \xi^{(t)}, \cdots, \xi^{(r)}\right): \xi^{(1)}, \cdots, \xi^{(s)}, C \cdot \xi^{(s+1)}, \cdots, \xi^{(t)}, D
\end{aligned}
$$

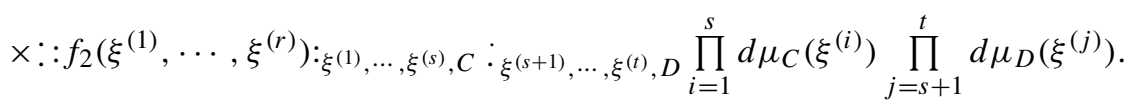

If $f_{1}$ has degree at least one in the variables $\xi^{(1)}, \cdots, \xi^{(s)}$ and degree at least three in the variables $\xi^{(1)}, \cdots, \xi^{(t)}$ then

$$
N_{\text {impr }}(g ; \alpha) \leq 27 \frac{J}{\alpha^{6}} N\left(f_{1} ; \alpha\right) N\left(f_{2} ; \alpha\right)
$$

for $\alpha \geq 2$.

Proof. Set $\tilde{f}_{i}=:: f_{i}: \xi^{(1)}, \cdots, \xi^{(s)}, C: \xi^{(s+1)}, \ldots, \xi^{(t)}, D$. We first prove the statement in the case that $f_{1}$ and $f_{2}$ are both homogeneous, that is

$$
\begin{aligned}
& f_{1} \in A_{0}\left[n_{1}, \cdots, n_{s}, \cdots, n_{t}, \cdots, n_{r}\right], \\
& f_{2} \in A_{0}\left[n_{1}^{\prime}, \cdots, n_{r}^{\prime}\right] .
\end{aligned}
$$

Then $g=0$ unless $n_{i}=n_{i}^{\prime}$ for $1 \leq i \leq t$, and $g \in A_{0}\left[n_{t+1}+n_{t+1}^{\prime}, \cdots, n_{r}+n_{r}^{\prime}\right]$. By hypothesis $n_{1}+\cdots+n_{s} \geq 1$ and $n_{1}+\cdots+n_{t} \geq 3$. Therefore it is possible to choose $i_{1} \in\{1, \cdots, s\}$ with $n_{i_{1}} \geq 1$, and to choose $i_{2}, i_{3} \in\{1, \cdots, t\}$ such that

$$
\begin{aligned}
& n_{i_{2}} \geq \begin{cases}1 & \text { if } i_{2} \neq i_{1} \\
2 & \text { if } i_{2}=i_{1}\end{cases} \\
& n_{i_{3}} \geq \begin{cases}1 & \text { if } i_{3} \neq i_{1}, i_{2} \\
2 & \text { if } i_{3} \in\left\{i_{1}, i_{2}\right\} \text { but } i_{1} \neq i_{2} . \\
3 & \text { if } i_{3}=i_{2}=i_{1}\end{cases}
\end{aligned}
$$

Set

$$
C_{v}^{\prime}=\left\{\begin{array}{ll}
C & \text { if } 1 \leq i_{v} \leq s \\
D & \text { if } s+1 \leq i_{v} \leq t
\end{array} .\right.
$$

Clearly, $C_{1}^{\prime}=C$. Also set $\operatorname{Con}_{v}=\underset{\xi^{\left(i_{v}\right)} \rightarrow \zeta^{\left(i_{v}\right)}}{\operatorname{Con}_{C^{\prime}}}$ and

$$
\begin{aligned}
g^{\prime}\left(\xi^{(1)}, \cdots, \xi^{(r)} ; \zeta^{(1)}, \cdots, \zeta^{(r)}\right)= & \operatorname{Con}_{1} \operatorname{Con}_{2} \operatorname{Con}_{3} f_{1}\left(\xi^{(1)}, \cdots, \xi^{(r)}\right) \\
& \times f_{2}\left(\zeta^{(1)}, \cdots, \zeta^{(r)}\right), \\
g^{\prime \prime}\left(\xi^{(1)}, \cdots, \xi^{(r)} ; \zeta^{(1)}, \cdots, \zeta^{(r)}\right)= & \operatorname{Con}_{1} \operatorname{Con}_{2} \operatorname{Con}_{3} \tilde{f}_{1}\left(\xi^{(1)}, \cdots, \xi^{(r)}\right) \\
& \times \tilde{f}_{2}\left(\zeta^{(1)}, \cdots, \zeta^{(r)}\right) .
\end{aligned}
$$


Observe that

$$
\begin{gathered}
g^{\prime} \in A_{0}^{\prime}\left[n_{1}-\left(\delta_{1 i_{1}}+\delta_{1 i_{2}}+\delta_{1 i_{3}}\right), \cdots, n_{r}-\left(\delta_{r i_{1}}+\delta_{r i_{2}}+\delta_{r i_{3}}\right),\right. \\
\left.n_{1}^{\prime}-\left(\delta_{1 i_{1}}+\delta_{1 i_{2}}+\delta_{1 i_{3}}\right), \cdots, n_{r}^{\prime}-\left(\delta_{r i_{1}}+\delta_{r i_{2}}+\delta_{r i_{3}}\right)\right]
\end{gathered}
$$

and

$$
\begin{aligned}
& g^{\prime \prime}\left(\xi^{(1)}, \cdots, \xi^{(r)} ; \zeta^{(1)}, \cdots, \zeta^{(r)}\right) \\
& =:: g^{\prime}\left(\xi^{(1)}, \cdots, \xi^{(r)} ; \zeta^{(1)}, \cdots, \zeta^{(r)}\right):_{\zeta^{(1)}, \ldots, \zeta^{(s)}, C} \underset{\zeta^{(s+1), \ldots, \zeta^{(t)}, D}}{ }
\end{aligned}
$$

by Remark II.12. By Lemma II.13,

$$
g=\iint g^{\prime \prime}\left(\xi^{(1)}, \cdots, \xi^{(r)} ; \xi^{(1)}, \cdots, \xi^{(r)}\right) \prod_{i=1}^{s} d \mu_{C}\left(\xi^{(i)}\right) \prod_{j=s+1}^{t} d \mu_{D}\left(\xi^{(j)}\right) .
$$

By Lemma II.29 and Lemma VI.2,

$$
\|g\|_{\text {impr }} \leq \mathrm{b}^{2\left(n_{1}+\cdots+n_{t}-3\right)}\left\|g^{\prime}\right\|_{\text {impr }} \leq J \frac{n_{i_{1}} n_{i_{2}} n_{i_{3}} \mathfrak{c}}{\mathrm{b}^{2}} \mathrm{~b}^{2\left(n_{1}+\cdots+n_{t}\right)}\left\|f_{1}\right\|\left\|f_{2}\right\| .
$$

Therefore

$$
\begin{aligned}
N_{\mathrm{impr}}(g) & =\frac{\mathfrak{c}}{\mathrm{b}^{2}} \alpha^{n_{t+1}+\cdots+n_{r}+n_{t+1}^{\prime}+\cdots+n_{r}^{\prime}} \mathbf{b}^{n_{t+1}+\cdots+n_{r}+n_{t+1}^{\prime}+\cdots+n_{r}^{\prime}}\|g\|_{\text {impr }} \\
& \leq \frac{n_{i_{1}} n_{i_{2}} n_{i_{3}}}{\alpha^{2\left(n_{1}+\cdots+n_{t}\right)}} \frac{J \mathfrak{c}^{2}}{\mathrm{~b}^{4}}(\alpha \mathrm{b})^{n_{1}+\cdots+n_{r}+n_{1}^{\prime}+\cdots+n_{r}^{\prime}}\left\|f_{1}\right\|\left\|f_{2}\right\| \\
& \leq J \frac{n_{i_{1}} n_{i_{2}} n_{i_{3}}}{\alpha^{2\left(n_{1}+\cdots+n_{t}\right)}} N\left(f_{1}\right) N\left(f_{2}\right) \\
& \leq 27 \frac{J}{\alpha^{6}} N\left(f_{1}\right) N\left(f_{2}\right) .
\end{aligned}
$$

The general case now follows by decomposing $f_{1}$ and $f_{2}$ into homogeneous pieces.

VI.2. Ladders. Theorem VI.6, below, which is the main result of this paper, shows that under appropriate assumptions on an effective interaction $W(\psi)$, the two point and non-ladder four point parts of the effective interaction : $W^{\prime}(\psi): \psi, D=\Omega_{C}(: W: \psi, C+D)$, constructed using the Grassmann Gaussian integral with covariance $C$, obeys estimates that are better by a factor $J$ than those one would expect from Theorem IV.1. To formulate this precisely, we first give the definition of ladders.

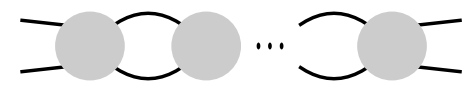

In a ladder, neighbouring four legged vertices are connected by two covariances. Since ladders result from integrating with covariance $C$, at least one of the connecting covariances is equal to $C$. The other connecting covariance may be $C$ or $D$.

In the rest of the paper, we will systematically use $\xi, \xi^{\prime}, \xi^{\prime \prime}, \cdots$ for fields associated to the covariance $C$. We will use $\zeta, \zeta^{\prime}, \zeta^{\prime \prime}, \cdots$ for fields associated to the covariance $D$ and $\psi$ for the external fields. 
Definition VI.5. (i) A rung is a Grassmann function

$\rho\left(\zeta, \xi ; \zeta^{\prime}, \xi^{\prime}\right) \in A[0,2,0,2] \oplus A[1,1,0,2] \oplus A[0,2,1,1] \oplus A[1,1,1,1]$.

We think of $\zeta, \xi$ as the $D$ resp. C fields on the left side of the rung and of $\zeta^{\prime}, \xi^{\prime}$ as the $D$ resp. $C$ fields on the right side of the rung.

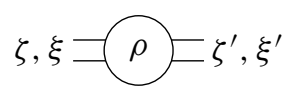

An end is a Grassmann function

$$
E(\psi ; \zeta, \xi) \in A[2,0,2] \oplus A[2,1,1] .
$$

We think of $\psi$ as the external fields at the end of the ladder and of $\zeta$, $\xi$ as the $D$ resp. C fields going into the ladder.

$$
\psi=\zeta, \xi
$$

(ii) If $E$ is an end and $\rho$ is a rung, we define the end $E \circ \rho$ by

$$
E \circ \rho\left(\psi ; \zeta^{\prime}, \xi^{\prime}\right)=\iint: E(\psi ; \zeta, \xi): \underset{\zeta, D}{\xi, C}: \rho\left(\zeta, \xi ; \zeta^{\prime}, \xi^{\prime}\right): \underset{\zeta, D}{\xi, C} d \mu_{C}(\xi) d \mu_{D}(\zeta)
$$

If $E_{1}, E_{2}$ are ends, we define the ladder $E_{1} \circ E_{2}$ by

$$
\begin{aligned}
& E_{1} \circ E_{2}(\psi)=\iint: E_{1}\left(\psi ; \zeta^{\prime}, \xi^{\prime}\right): \underset{\substack{\xi^{\prime}, C \\
\zeta^{\prime}, D}}{ }: E_{2}\left(\psi ; \zeta^{\prime}, \xi^{\prime}\right): \underset{\substack{\xi^{\prime}, C \\
\zeta^{\prime}, D}}{ } d \mu_{C}\left(\xi^{\prime}\right) d \mu_{D}\left(\zeta^{\prime}\right) \\
& \psi=E_{1} \frac{E_{2}}{\zeta^{\prime}, \xi^{\prime}}=\psi
\end{aligned}
$$

(iii) Let $F(\xi) \in A[4]$. Write

$$
\begin{aligned}
F\left(\xi^{(1)}+\xi^{(2)}+\xi^{(3)}\right) & =\sum_{n_{1}+n_{2}+n_{3}=4} F_{n_{1}, n_{2}, n_{3}}\left(\xi^{(1)}, \xi^{(2)}, \xi^{(3)}\right), \\
F\left(\xi^{(1)}+\xi^{(2)}+\xi^{(3)}+\xi^{(4)}\right) & =\sum_{n_{1}+n_{2}+n_{3}+n_{4}=4} F_{n_{1}, n_{2}, n_{3}, n_{4}}\left(\xi^{(1)}, \xi^{(2)}, \xi^{(3)}, \xi^{(4)}\right)
\end{aligned}
$$

with $F_{n_{1}, n_{2}, n_{3}} \in A\left[n_{1}, n_{2}, n_{3}\right]$ and $F_{n_{1}, n_{2}, n_{3}, n_{4}} \in A\left[n_{1}, n_{2}, n_{3}, n_{4}\right]$. The rung associated to $F$ is

$$
\rho(F)\left(\zeta, \xi ; \zeta^{\prime}, \xi^{\prime}\right)=F_{0,2,0,2}+F_{1,1,0,2}+F_{0,2,1,1}+F_{1,1,1,1} .
$$

The end associated to $F$ is

$$
E(F)\left(\psi ; \zeta^{\prime}, \xi^{\prime}\right)=F_{2,0,2}+F_{2,1,1}
$$


The ladder of length $r \geq 1$ with vertex $F$ is defined as

$$
L_{r}(F)(\psi)=E(F) \circ \rho(F) \circ \rho(F) \circ \cdots \rho(F) \circ E(F)
$$

with $(r-1)$ copies of $\rho(F)$.

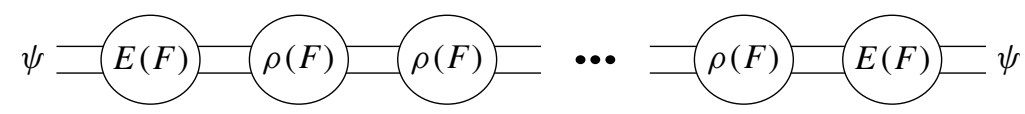

In Appendix C, we describe ladders in terms of kernels.

The main result of this paper is

Theorem VI.6. Let $W(\psi)$ be an even Grassmann function with coefficients in A. Assume that $N(W ; 64 \alpha)_{0}<\frac{1}{8} \alpha$, and that $\alpha \geq 8$. Set

$$
: W^{\prime}(\psi): \psi, D=\Omega_{C}(: W: \psi, C+D)
$$

If $(C, D)$ have improved integration constants $\mathfrak{c}, \mathrm{b}, J$, then

(i)

$$
\begin{aligned}
N\left(W^{\prime}-W ; \alpha\right) & \leq \frac{1}{2 \alpha^{2}} \frac{N(W ; 32 \alpha)^{2}}{1-\frac{1}{\alpha^{2}} N(W ; 32 \alpha)}, \\
N_{\text {impr }}\left(W^{\prime}-W ; \alpha\right) & \leq \frac{1}{2 \alpha^{2}} \frac{N(W ; 32 \alpha)^{2}}{1-\frac{1}{\alpha^{2}} N(W ; 32 \alpha)} .
\end{aligned}
$$

(ii) Write $W(\psi)=\sum_{m ; n} W_{m ; n}(\psi), W^{\prime}(\psi)=\sum_{m ; n} W_{m ; n}^{\prime}(\psi)$ with $W_{m ; n}, W_{m ; n}^{\prime} \in$ $A_{m}^{\prime}[n]$. If $W_{0 ; 2}=0$, then

$$
\begin{aligned}
N_{\text {impr }}\left(W_{0,2}^{\prime} ; \alpha\right) & \leq \frac{2^{10} J}{\alpha^{6}} \frac{N(W ; 64 \alpha)^{2}}{1-\frac{8}{\alpha} N(W ; 64 \alpha)}, \\
N_{\text {impr }}\left(W_{0,4}^{\prime}-W_{0,4}-\frac{1}{2} \sum_{r=1}^{\infty} L_{r}\left(W_{0,4}\right) ; \alpha\right) & \leq \frac{2^{10} J}{\alpha^{6}} \frac{N(W ; 64 \alpha)^{2}}{1-\frac{8}{\alpha} N(W ; 64 \alpha)} .
\end{aligned}
$$

Remark VI.7. i) Part (i) of the theorem follows directly from Theorem IV.1. For the proof of part (ii) one can replace the algebra $A$ by $A_{0}$, since $W_{0,2}^{\prime}, W_{0,2}^{\prime}$ and $L_{r}\left(W_{0,4}\right)$ depend only on $\sum_{n=0}^{\infty} W_{0 ; n}$.

ii) The hypothesis that $W_{0 ; 2}=0$ in part (ii) of Theorem VI.6 prevents strings of twolegged vertices from appearing in diagrammatic expansions. The expansion used in the proof of part (ii) cannot detect certain overlapping loops containing such strings. In practice a nonzero $W_{0 ; 2}$ can be absorbed in the propagator. 
The proof of part (ii) of Theorem VI.6 is based on an analysis of

VI.3. Overlapping Loops for the Schwinger Functional. We first generalise the concept of a ladder. If $U(\psi ; \xi)$ is a Grassmann function we write

$$
U\left(\psi+\zeta+\zeta^{\prime} ; \xi+\xi^{\prime}\right)=\sum_{\substack{p_{1}, p_{2} \\ n_{0}, n_{1}, n_{2}}} U_{n_{0} ; p_{1}, p_{2} ; n_{1}, n_{2}}\left(\psi ; \zeta, \zeta^{\prime} ; \xi, \xi^{\prime}\right)
$$

with $U_{n_{0} ; p_{1}, p_{2} ; n_{1}, n_{2}} \in A\left[n_{0} ; p_{1}, p_{2}, n_{1}, n_{2}\right]$.

Definition VI.8. Let $U$ be as above.

(i) The rung associated to $U$ is

$$
\operatorname{Rung}(U)\left(\zeta, \xi ; \zeta^{\prime}, \xi^{\prime}\right)=U_{0 ; 0,2 ; 0,2}+U_{0 ; 1,1 ; 0,2}+U_{0 ; 0,2 ; 1,1}+U_{0 ; 1,1 ; 1,1}
$$

(ii) The tail $T_{\ell}(U)$ associated to $U$ is recursively defined as

$$
\begin{aligned}
T_{1}(U)\left(\psi ; \zeta^{\prime}, \xi^{\prime}\right) & =U_{2 ; 0,0 ; 0,2}+U_{2 ; 0,1 ; 0,1}, \\
T_{\ell+1}(U)\left(\psi ; \zeta^{\prime}, \xi^{\prime}\right) & =T_{\ell}(U) \circ \operatorname{Rung}(U) .
\end{aligned}
$$

Observe that $T_{\ell}(U)\left(\psi ; \zeta^{\prime}, \xi^{\prime}\right)$ lies in $A[2,0,2] \oplus A[2,1,1]$.

Later we need

Remark VI.9. Let $E_{1}, E_{2}$ be ends whose coefficients are even elements of $A$ and let $g(\psi ; \xi)$ be a Grassmann function. Set

$$
\begin{aligned}
& h(\psi)=\iint: E_{1}\left(\psi ; \zeta^{\prime}, \xi^{\prime}\right) E_{2}\left(\psi ; \zeta^{\prime}, \xi^{\prime}\right):_{\substack{\zeta^{\prime}, D \\
\xi^{\prime}, C}}: g\left(\psi+\zeta^{\prime} ; \xi^{\prime}\right):_{\substack{\zeta^{\prime}, D \\
\xi^{\prime}, C}} d \mu_{D}\left(\zeta^{\prime}\right) d \mu_{C}\left(\xi^{\prime}\right), \\
& h(\psi)=\sum_{n=4}^{\infty} h_{n}(\psi) \quad \text { with } h_{n} \in A[n] .
\end{aligned}
$$

Then

$$
h_{4}=E_{1} \circ \operatorname{Rung}(g) \circ E_{2} .
$$

Proof. By Lemma A.5,

$$
\begin{aligned}
& h(\psi)=\iint: E_{1}(\psi ; \zeta, \xi):_{\substack{\xi, D \\
\xi, C}}: g\left(\psi+\zeta+\zeta^{\prime} ; \xi+\xi^{\prime}\right):_{\substack{\zeta, \zeta^{\prime}, D \\
\xi^{\prime}, \xi^{\prime}, C}} \\
& : E_{2}\left(\psi ; \zeta^{\prime}, \xi^{\prime}\right):_{\substack{\zeta^{\prime}, D \\
\xi^{\prime}, C}} d \mu_{D}\left(\zeta, \zeta^{\prime}\right) d \mu_{C}\left(\xi, \xi^{\prime}\right) .
\end{aligned}
$$

As $E_{1}$ is of degree at most one in $\zeta$ and $E_{2}$ is of degree at most one in $\zeta^{\prime}$,

$$
\begin{aligned}
h_{4}(\psi)= & \iint: E_{1}(\psi ; \zeta, \xi): \xi, C \\
& \left.: \operatorname{Rung}(g)\left(\zeta, \xi ; \zeta^{\prime}, \xi^{\prime}\right): \xi ; \zeta^{\prime}, \xi^{\prime}\right): \xi^{\prime}, C \\
= & \left(E_{1} \circ \operatorname{Rung}(g) \circ \mu_{D}\left(\zeta, \zeta^{\prime}\right) d \mu_{C}\left(\xi, \xi^{\prime}\right)\right.
\end{aligned}
$$


The main estimate on the Schwinger functional is:

Theorem VI.10. Let A be a superalgebra, with all elements having degree zero (that is $\left.A=A_{0}\right),\|\cdot\|$ and $\|\cdot\|_{\mathrm{impr}}$ be two families of symmetric seminorms on the spaces $A_{m} \otimes$ $V^{\otimes n}$ and let $(C, D)$ have improved integration constants $\mathfrak{c}, \mathrm{b}, J$. Let $\hat{U}(\psi, \xi), \hat{f}(\psi, \xi)$ be Grassmann functions with coefficients in A of degree at least four and $\hat{U}$ even. Set

$$
\begin{aligned}
U(\psi, \xi) & =: \hat{U}(\psi, \xi): \underset{\xi, D}{\psi, D}, \\
f(\psi, \xi) & =: \hat{f}(\psi, \xi): \underset{\xi, D}{\psi, D} .
\end{aligned}
$$

Assume that $\alpha \geq 8$ and $N(\hat{U} ; 32 \alpha)_{0}<\frac{1}{8} \alpha$. By Proposition III.10,

$$
: f^{\prime}(\psi):_{\psi, D}=\mathcal{S}_{U, C}(f)
$$

exists. Write

$$
\hat{f}(\psi, \xi)=\sum_{n_{0}, n_{1}} \hat{f}_{n_{0}, n_{1}}(\psi, \xi), \quad f^{\prime}(\psi)=\sum_{n} f_{n}^{\prime}(\psi)
$$

with $\hat{f}_{n_{0}, n_{1}} \in A\left[n_{0}, n_{1}\right], f_{n}^{\prime} \in A[n]$. Then

$$
N_{\text {impr }}\left(f_{2}^{\prime} ; \alpha\right) \leq \frac{2^{10} J}{\alpha^{6}} \frac{N(\hat{U} ; 32 \alpha)}{1-\frac{8}{\alpha} N(\hat{U} ; 32 \alpha)} N(\hat{f} ; 32 \alpha)
$$

and there exists a Grassmann function $g(\psi)$ such that

$$
f_{4}^{\prime}=\hat{f}_{4,0}+\sum_{\ell=1}^{\infty} T_{\ell}(\hat{U}) \circ T_{1}(\hat{f})+\frac{1}{2} \sum_{\ell, \ell^{\prime} \geq 1} T_{\ell}(\hat{U}) \circ \operatorname{Rung}(\hat{f}) \circ T_{\ell^{\prime}}(\hat{U})+g
$$

and

$$
N_{\text {impr }}(g ; \alpha) \leq \frac{2^{10} J}{\alpha^{6}} \frac{N(\hat{U} ; 32 \alpha)}{1-\frac{8}{\alpha} N(\hat{U} ; 32 \alpha)} N(\hat{f} ; 32 \alpha) .
$$

In the case $D=0$ this theorem is proven in Sect. VI; in the general case it is proven in Sect. VIII.

Proof that Theorem VI.10 implies Theorem VI.6. By part (i) of Remark VI.7 we may assume that $A=A_{0}$. We write $W_{n}$ for $W_{0 ; n}$ and $W_{n}^{\prime}$ for $W_{0 ; n}^{\prime}$. Set

$$
\begin{aligned}
\hat{U}(\psi, \xi) & =W(\psi+\xi) \in \bigwedge_{A^{\prime}} V, \\
U(\psi, \xi) & =: \hat{U}(\psi, \xi):{ }_{\xi, C}^{\psi, D}, \\
: U_{t}^{\prime}(\psi): \psi, D & =\mathcal{S}_{t U, C}(U) .
\end{aligned}
$$

By Remark II.24, $N(\hat{U} ; \alpha) \leq N(W ; 2 \alpha)$. As in the proof of Theorem II.28,

$$
: W^{\prime}(\psi):_{\psi, D}-: W(\psi):_{\psi, D}=\int_{0}^{1}\left(: U_{t}^{\prime}(\psi):_{\psi, D}-: \hat{U}(\psi, 0):_{\psi, D}\right) d t \quad \bmod A_{0}
$$

so

$$
W^{\prime}(\psi)-W(\psi)=\int_{0}^{1}\left(U_{t}^{\prime}(\psi)-\hat{U}(\psi, 0)\right) d t \quad \bmod A_{0}
$$


In particular, for $n=2,4$,

$$
W_{n}^{\prime}-W_{n}=\int_{0}^{1}\left(U_{t, n}^{\prime}-\hat{U}_{n, 0}\right) d t .
$$

Therefore, by Theorem VI.10,

$$
\begin{aligned}
N_{\text {impr }}\left(W_{2}^{\prime}\right) & \leq \max _{0 \leq t \leq 1} N_{\text {impr }}\left(U_{t, 2}^{\prime}\right) \\
& \leq \frac{2^{10} J}{\alpha^{6}} \frac{N(\hat{U} ; 32 \alpha)}{1-\frac{8}{\alpha} N(\hat{U} ; 32 \alpha)} N(\hat{U} ; 32 \alpha) \\
& \leq \frac{2^{10} J}{\alpha^{6}} \frac{N(W ; 64 \alpha)^{2}}{1-\frac{8}{\alpha} N(W ; 64 \alpha)} .
\end{aligned}
$$

Observe that

$$
\begin{aligned}
\operatorname{Rung}(\hat{U}) & =\rho\left(W_{4}\right), \\
T_{\ell}(\hat{U}) & =E\left(W_{4}\right) \circ \rho\left(W_{4}\right) \circ \cdots \circ \rho\left(W_{4}\right)
\end{aligned}
$$

with $\ell-1$ copies of $\rho\left(W_{4}\right)$. Therefore

$$
\begin{aligned}
T_{\ell}(t \hat{U}) \circ T_{1}(\hat{U}) & =t^{\ell} L_{\ell}\left(W_{4}\right), \\
T_{\ell}(t \hat{U}) \circ \operatorname{Rung}(\hat{U}) \circ T_{\ell^{\prime}}(t \hat{U}) & =t^{\ell+\ell^{\prime}} L_{\ell+\ell^{\prime}}\left(W_{4}\right) .
\end{aligned}
$$

Hence, by Theorem VI.10,

$$
W_{4}^{\prime}=W_{4}+\sum_{\ell=1}^{\infty} \int_{0}^{1} t^{\ell} L_{\ell}\left(W_{4}\right) d t+\frac{1}{2} \sum_{\ell, \ell^{\prime} \geq 1} \int_{0}^{1} t^{\ell+\ell^{\prime}} L_{\ell+\ell^{\prime}}\left(W_{4}\right) d t+g
$$

with

$$
N_{\text {impr }}(g) \leq \frac{2^{10} J}{\alpha^{6}} \frac{N(\hat{U} ; 32 \alpha)}{1-\frac{8}{\alpha} N(\hat{U} ; 32 \alpha)} N(\hat{U} ; 32 \alpha) \leq \frac{2^{10} J}{\alpha^{6}} \frac{N(W ; 64 \alpha)^{2}}{1-\frac{8}{\alpha} N(W ; 64 \alpha)}
$$

Now

$$
\begin{aligned}
\sum_{\ell=1}^{\infty} \int_{0}^{1} t^{\ell} L_{\ell}\left(W_{4}\right) d t+\frac{1}{2} \sum_{\ell, \ell^{\prime} \geq 1} \int_{0}^{1} t^{\ell+\ell^{\prime}} L_{\ell+\ell^{\prime}}\left(W_{4}\right) d t & =\frac{1}{2} \sum_{r=1}^{\infty} \int_{0}^{1}(r+1) t^{r} L_{r}\left(W_{4}\right) d t \\
& =\frac{1}{2} \sum_{r=1}^{\infty} L_{r}\left(W_{4}\right) .
\end{aligned}
$$

VI.4. Configurations of Norms with Improved Power Counting.

Definition VI.11. Let $q$ be an even natural number. For $p=1,2,3, \cdots, q$, let $\|\cdot\|_{p}$ be a system of symmetric seminorms on the spaces $A_{m} \otimes V^{\otimes n}$. We say that $(C, D)$ have integration constants $\mathfrak{c}, \mathrm{b}$ for the configuration $\|\cdot\|_{1},\|\cdot\|_{2}, \cdots,\|\cdot\|_{q}$ of seminorms if the following estimates hold:

Let $m, m^{\prime} \geq 0$ and $1 \leq i \leq n, 1 \leq j \leq n^{\prime}$. Also let $f \in A_{m} \otimes V^{\otimes n}, f^{\prime} \in A_{m^{\prime}} \otimes V^{\otimes n^{\prime}}$. Then for all natural numbers $p \leq q$ the simple contraction estimate

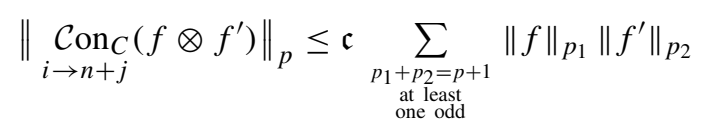

holds. 
Furthermore, if $C_{2}, C_{3} \in\{C, D\}, m=m^{\prime}=0,1 \leq i_{1}, i_{2}, i_{3} \leq n$ with $i_{1}, i_{2}, i_{3}$ all different and $1 \leq j_{1}, j_{2}, j_{3} \leq n^{\prime}$ with $j_{1}, j_{2}, j_{3}$ all different, the improved contraction estimate

$$
\left\|\underset{i_{1} \rightarrow n+j_{1}}{\mathcal{C} \operatorname{Con}_{C}} \underset{i_{2} \rightarrow n+j_{2}}{\mathcal{C o n}_{C_{2}}} \underset{i_{3} \rightarrow n+j_{3}}{\mathcal{C o n}_{C_{3}}}\left(f \otimes f^{\prime}\right)\right\|_{p} \leq \mathrm{b}^{4} \mathfrak{c} \sum_{\substack{p_{1}+p_{2}=p+3 \\ \text { at least } \\ \text { one odd }}}\|f\|_{p_{1}}\left\|f^{\prime}\right\|_{p_{2}}
$$

holds for $p \leq q-2$.

For every $f \in A_{m} \otimes V^{\otimes n}$ and every $n^{\prime} \leq n$ the modified integral bound

$$
\left\|\int A n t_{n^{\prime}}(f) d \mu_{C}\right\|_{p},\left\|\int A n t_{n^{\prime}}(f) d \mu_{D}\right\|_{p} \leq \frac{1}{2}(\mathrm{~b} / 2)^{n^{\prime}}\left[\|f\|_{p}+\|f\|_{p-(-1)^{p}}\right]
$$

holds. The partial antisymmetrization Ant $n_{n^{\prime}}$ was defined in Def. II.25.ii.

Lemma VI.12. Let $q$ be an even natural number. Assume that $(C, D)$ have integration constants $\mathrm{c}, \mathrm{b}$ for the configuration $\|\cdot\|_{1},\|\cdot\|_{2}, \cdots,\|\cdot\|_{q}$ of seminorms and let $J>0$. For $f \in A_{m} \otimes V^{\otimes n}$, set

$$
\begin{aligned}
\|f\|= & \sum_{p=1}^{q} J^{-[(p-1) / 2]}\|f\|_{p}=\|f\|_{1}+\|f\|_{2}+\frac{1}{J}\|f\|_{3} \\
& +\frac{1}{J}\|f\|_{4}+\cdots+\frac{1}{J^{(q-2) / 2}}\|f\|_{q}, \\
\|f\|_{\text {impr }}= & \begin{cases}\sum_{p=1}^{q-2} J^{-[(p-1) / 2]}\|f\|_{p} & \text { if } m=0 \\
0 & \text { if } m \neq 0\end{cases}
\end{aligned}
$$

Here $[(p-1) / 2]$ is the integer part of $\frac{p-2}{2}$. Then $(C, D)$ have improved integration constants $\mathfrak{c}, \mathrm{b}, J$ for the families $\|\cdot\|$ and $\|\cdot\|_{\text {impr }}$ of seminorms.

Proof. Clearly $\|\cdot\|_{\text {impr }} \leq\|\cdot\|$. To verify that $\mathfrak{c}$ is a contraction bound for $C$, let $f \in A_{m} \otimes V^{\otimes n}, f^{\prime} \in A_{m^{\prime}} \otimes V^{\otimes n^{\prime}}$ and $1 \leq i \leq n, 1 \leq j \leq n^{\prime}$. Observe that if $p_{1}+p_{2}=p+1$ with at least one of $p_{1}$ and $p_{2}$ odd, then

$$
\frac{1}{J^{\left[\left(p_{1}-1\right) / 2\right]}} \frac{1}{J^{\left[\left(p_{2}-1\right) / 2\right]}}=\frac{1}{J^{\left[\left(p_{1}+p_{2}-2\right) / 2\right]}}=\frac{1}{J^{[(p-1) / 2]}} .
$$

Consequently,

$$
\begin{aligned}
& \left\|\underset{i \rightarrow n+j}{\mathcal{C o n}_{C}}\left(f \otimes f^{\prime}\right)\right\|=\sum_{p=1}^{q} J^{-[(p-1) / 2]}\left\|\underset{i \rightarrow n+j}{\mathcal{C o n}_{C}} f \otimes f^{\prime}\right\|_{p} \\
& \leq \sum_{p=1}^{q} \mathfrak{c} \sum_{\substack{p_{1}+p_{2}=p+1 \\
\text { at least } \\
\text { one odd }}} J^{-[(p-1) / 2]}\|f\|_{p_{1}}\left\|f^{\prime}\right\|_{p_{2}} \\
& \leq \sum_{p=1}^{q} \mathfrak{c} \sum_{p_{1}+p_{2}=p+1} J^{-\left[\left(p_{1}-1\right) / 2\right]}\|f\|_{p_{1}} J^{-\left[\left(p_{2}-1\right) / 2\right]}\left\|f^{\prime}\right\|_{p_{2}} \\
& \leq \mathfrak{c}\|f\|\left\|f^{\prime}\right\| \text {. }
\end{aligned}
$$


Replacing $q$ by $q-2$ gives the corresponding bound for $\|\cdot\|_{\text {impr }}$. To verify the triple contraction estimate of Def. VI.1, let $C_{2}, C_{3} \in\{C, D\}, m=m^{\prime}=0,1 \leq i_{1}, i_{2}, i_{3} \leq n$ with $i_{1}, i_{2}, i_{3}$ all different and $1 \leq j_{1}, j_{2}, j_{3} \leq n^{\prime}$ with $j_{1}, j_{2}, j_{3}$ all different. Then

$$
\begin{aligned}
& \left\|\underset{i_{1} \rightarrow n+j_{1}}{\mathcal{C o n}_{C}} \underset{i_{2} \rightarrow n+j_{2}}{\mathcal{C o n}_{C_{2}}} \underset{i_{3} \rightarrow n+j_{3}}{\mathcal{C o n}_{C_{3}}}\left(f \otimes f^{\prime}\right)\right\|_{\text {impr }}=\sum_{p=1}^{q-2} J^{-\left[\frac{p-1}{2}\right]} \| \underset{i_{1} \rightarrow n+j_{1}}{\mathcal{C o n}_{C}} \underset{i_{2} \rightarrow n+j_{2}}{\mathcal{C o n}_{C_{2}}} \\
& \times \underset{i_{3} \rightarrow n+j_{3}}{\mathcal{C o n}_{C_{3}}}\left(f \otimes f^{\prime}\right) \|_{p} \\
& \leq J \mathrm{~b}^{4} \mathfrak{c} \sum_{p=1}^{q-2} \sum_{\substack{p_{1}+p_{2}=p+3 \\
\text { at least } \\
\text { one odd }}}^{q} J^{-[(p+1) / 2]} \\
& \times\|f\|_{p_{1}}\left\|f^{\prime}\right\|_{p_{2}} \\
& \leq J \mathrm{~b}^{4} \mathfrak{c} \sum_{p_{1}, p_{2}=1}^{q} J^{-\left[\left(p_{1}-1\right) / 2\right]}\|f\|_{p_{1}} \\
& \times J^{-\left[\left(p_{2}-1\right) / 2\right]}\left\|f^{\prime}\right\|_{p_{2}} \\
& =J \mathrm{~b}^{4} \mathfrak{c}\|f\|\left\|f^{\prime}\right\| \text {. }
\end{aligned}
$$

We verify that $\mathrm{b}$ is an integral bound for $C$ for the norm $\|\cdot\|$. The other cases are similar. Let $f \in A_{m} \otimes V^{\otimes n}$ and $n^{\prime} \leq n$. Then

$$
\begin{aligned}
\left\|\int A n t_{n^{\prime}}(f) d \mu_{C}\right\| & =\sum_{p=1}^{q} J^{-[(p-1) / 2]}\left\|\int A n t_{n^{\prime}}(f) d \mu_{C}\right\|_{p} \\
& =\frac{1}{2}(\mathrm{~b} / 2)^{n^{\prime}} \sum_{p=1}^{q} J^{-[(p-1) / 2]}\left[\|f\|_{p}+\|f\|_{p-(-1)^{p}}\right] \\
& \leq(\mathrm{b} / 2)^{n^{\prime}}\|f\| .
\end{aligned}
$$

In our main application, we use a special case of Def. VI.11 in which only norms $\|\cdot\|_{p}$, with $p$ odd, appear.

Definition VI.13. Let $q$ be an odd natural number. For $p=1,3,5, \cdots, q$, let $\|\cdot\|_{p}$ be a system of symmetric seminorms on the spaces $A_{m} \otimes V^{\otimes n}$. We say that $(C, D)$ have integration constants $\mathfrak{c}, \mathrm{b}$ for the configuration $\|\cdot\|_{1},\|\cdot\|_{3}, \cdots,\|\cdot\|_{q}$ of seminorms if $\mathrm{b}$ is an integral bound for both $C$ and $D$ and all of the seminorms $\|\cdot\|_{p}$ (see Def. II.25.ii) and the following contraction estimates hold:

Let $m, m^{\prime} \geq 0$ and $1 \leq i \leq n, 1 \leq j \leq n^{\prime}$. Also let $f \in A_{m} \otimes V^{\otimes n}, f^{\prime} \in A_{m^{\prime}} \otimes V^{\otimes n^{\prime}}$ Then for all odd natural numbers $p \leq q$,

$$
\left\|\underset{i \rightarrow n+j}{\mathcal{C} \mathrm{Oon}_{C}}\left(f \otimes f^{\prime}\right)\right\|_{p} \leq \mathfrak{c} \sum_{\substack{p_{1}+p_{2}=p+1 \\ p_{1}, p_{2} \text { odd }}}\|f\|_{p_{1}}\left\|f^{\prime}\right\|_{p_{2}} .
$$

Furthermore, if $C_{2}, C_{3} \in\{C, D\}, m=m^{\prime}=0,1 \leq i_{1}, i_{2}, i_{3} \leq n$ with $i_{1}, i_{2}, i_{3}$ all different and $1 \leq j_{1}, j_{2}, j_{3} \leq n^{\prime}$ with $j_{1}, j_{2}, j_{3}$ all different, then, for all odd $p \leq q-2$,

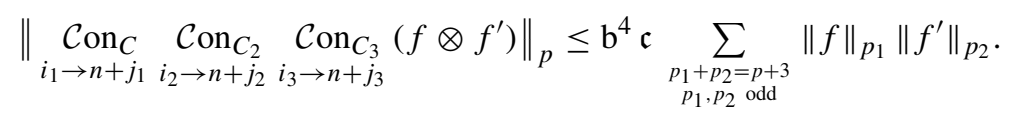


Remark VI.14. If, in the setting of Def. VI.13, the norm $\|\cdot\|_{p}$ is defined to be zero for all even $p$, then the conditions of Def. VI.11 are fulfilled, except that the factor of $\frac{1}{2}$ in (VI.2) does not appear in Def. II.25.ii of integral bound.

Lemma VI.15. Let $q$ be an odd natural number. Assume that $(C, D)$ have integration constants $\mathfrak{c}, \mathrm{b}$ for the configuration $\|\cdot\|_{1},\|\cdot\|_{3}, \cdots,\|\cdot\|_{q}$ of seminorms and let $J>0$. For $f \in A_{m} \otimes V^{\otimes n}$, set

$$
\begin{aligned}
\|f\| & =\sum_{\substack{p=1 \\
p \text { odd }}}^{q} J^{(1-p) / 2}\|f\|_{p}, \\
\|f\|_{\text {impr }} & = \begin{cases}\sum_{p=1}^{q-2} J^{(1-p) / 2}\|f\|_{p} & \text { if } m=0 \\
p \text { odd } & \text { if } m \neq 0 \\
0 & \end{cases}
\end{aligned}
$$

Then $(C, D)$ have improved integration constants $\mathfrak{c}, \mathrm{b}, J$ for the families $\|\cdot\|$ and $\|\cdot\|_{\text {impr }}$ of seminorms.

Proof. By Remark VI.14, Lemma VI.12 implies all of the conditions of Def. VI.1, except that $\mathrm{b}$ be an integral bound for $C$ and $D$ for both seminorms. However, the proof of this condition is virtually identical to (VI.3).

Remark VI.16. Lemma VI.15 holds for all $J>0$. In applications, $J$ is chosen so that

$$
\|f\|_{p} \leq \text { const } J^{(p-1) / 2}\|f\|_{1}
$$

for all $f$ of interest. If $J$ satisfying (VI.4) can be chosen sufficiently small, Lemma VI.15 can be used in conjunction with Prop. VI.4 to obtain improved bounds, as the following example illustrates.

For simplicity, we assume that $q=3$. Let $f\left(\xi^{(1)}, \xi^{(2)}\right) \in A_{0}\left[n_{1}, n_{2}\right]$ with $n_{2} \geq 3$ and set

$$
g\left(\xi^{(1)}\right)=\int\left(: f\left(\xi^{(1)}, \xi^{(2)}\right):_{\xi^{(2)}, C}\right)^{2} d \mu_{C}\left(\xi^{(2)}\right)
$$

The standard bound, without improvement, follows from (VI.4) in the proof of Prop. II.33:

$$
\|g\|_{1} \leq n_{2} \mathrm{cb}^{2\left(n_{2}-1\right)}\|f\|_{1}^{2} .
$$

On the other hand, by (VI.1), in the proof of Prop. VI.4,

$$
\|g\|_{1}=\|g\|_{\text {impr }} \leq n_{2}^{3} J \mathfrak{c b}^{2\left(n_{2}-1\right)}\|f\|^{2}=n_{2}^{3} J \mathfrak{c b}^{2\left(n_{2}-1\right)}\left(\|f\|_{1}+\frac{1}{J}\|f\|_{3}\right)^{2} .
$$

If $\|f\|_{3} \leq$ const $J\|f\|_{1}$,

$$
\|g\|_{1} \leq \text { const } n_{2}^{3} J \mathfrak{c b}^{2\left(n_{2}-1\right)}\|f\|_{1}^{2} .
$$




\section{Finding Overlapping Loops}

In this chapter, we give the proof of Theorem VI.10 in the case $D=0$, using the representation $\mathcal{S}_{U, C}=\int \frac{1}{1-R_{U, C}} d \mu_{C}$ of Theorem III.2. We assume that the coefficient algebra $A$ contains only elements of degree zero, that is $A=A_{0}$. Let $\|\cdot\|$ and $\|\cdot\|_{\text {impr }}$ be two families of symmetric seminorms on the spaces $A_{m} \otimes V^{\otimes n}$ such that $(C, 0)$ has improved integration constants $\mathfrak{c}, \mathrm{b}, J$ for these families of seminorms.

Recall from Remark III.6 that the operator $\mathcal{R}_{K, C}$ is written as a sum of operators $R_{C}\left(K_{1}, \cdots, K_{\ell}\right)$ with even Grassmann functions $K_{1}\left(\xi, \xi^{\prime}, \eta\right), \cdots, K_{\ell}\left(\xi, \xi^{\prime}, \eta\right)$. If one of these Grassmann functions, say $K_{1}$ has degree at least three in the variables $\xi^{\prime}, \eta$ then, for any Grassmann function $f(\xi)$, there is a pair of overlapping loops in each Feynman diagram contributing to $R_{C}\left(K_{1}, \cdots, K_{\ell}\right)(: f:)$. The way these overlapping loops can occur is indicated in the figures below.

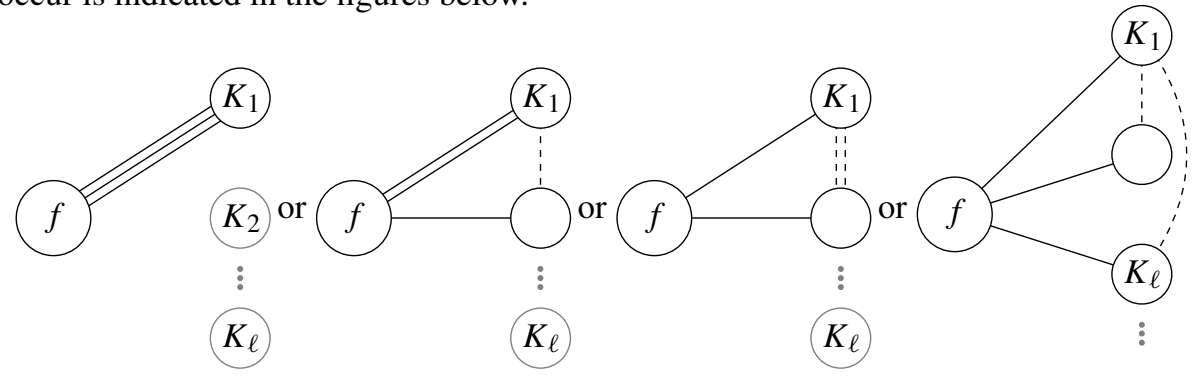

VII.1. Overlapping Loops Created by the Operator $\mathcal{R}_{K, C}$. In this subsection, we suppress the external fields $\psi$ by working in the Grassmann algebra $\bigwedge_{A^{\prime}} V$ with coefficients in the algebra $A^{\prime}=\bigwedge_{A} V$ generated by the fields $\psi$. This algebra was defined in Subsect. III.2. Recall that $\|\cdot\|$ and $\|\cdot\|_{\text {impr }}$ induce a family of symmetric seminorms on the spaces $A_{m}^{\prime} \otimes V^{\otimes n}$, which we here denote by the same symbols.

We split up the operators $R_{C}\left(K_{1}, \cdots, K_{\ell}\right)$ of (III.2) in order to exhibit possible overlapping loops. For Grassmann functions $K_{2}\left(\xi, \xi^{\prime}, \eta\right), \cdots, K_{\ell}\left(\xi, \xi^{\prime}, \eta\right)$ and $f(\xi)$ we define

$$
\begin{aligned}
\tilde{R}_{C}\left(K_{2}, \cdots, K_{\ell}\right)(f)= & \iint:\left(\prod_{i=2}^{\ell}: K_{i}\left(\xi, \xi^{\prime}+\xi^{\prime \prime}, \eta^{\prime}\right): \xi^{\prime \prime}\right):_{\eta^{\prime}} \\
& \times f\left(\eta+\eta^{\prime}\right) d \mu_{C}\left(\xi^{\prime \prime}\right) d \mu_{C}\left(\eta^{\prime}\right) .
\end{aligned}
$$

This is a Grassmann function of $\xi, \xi^{\prime}, \eta$ that is schematically represented in the figure below.

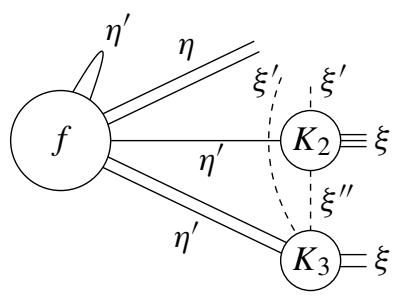


Proposition VII.1. For even Grassmann functions $K_{1}\left(\xi, \xi^{\prime}, \eta\right), \cdots, K_{\ell}\left(\xi, \xi^{\prime}, \eta\right)$ and $f(\xi)$

$$
\begin{aligned}
& R_{C}\left(K_{1}, \cdots, K_{\ell}\right)(f)=: \iint: K_{1}\left(\xi, \xi^{\prime}, \eta\right): \xi^{\prime}, \eta: \tilde{R}_{C}\left(K_{2}, \cdots, K_{\ell}\right) \\
& \times(f)\left(\xi, \xi^{\prime}, \eta\right): \xi^{\prime}, \eta d \mu_{C}\left(\xi^{\prime}\right) d \mu_{C}(\eta):_{\xi},
\end{aligned}
$$

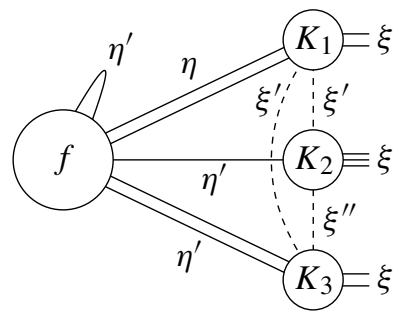

Proof. If

$$
: f^{\prime}: \xi=R_{C}\left(K_{1}, \cdots, K_{\ell}\right)(f),
$$

then by part (iii) of Prop. A.2 (applied to the variable $\xi^{\prime}$ ) and Lemma A.5 (applied to the variable $\eta$ )

$$
\begin{aligned}
f^{\prime}(\xi)= & \iint:\left[: K_{1}\left(\xi, \xi^{\prime}, \eta\right): \xi^{\prime} \prod_{i=2}^{\ell}: K_{i}\left(\xi, \xi^{\prime}, \eta\right): \xi^{\prime}\right]:_{\eta} f(\eta) d \mu_{C}\left(\xi^{\prime}\right) d \mu_{C}(\eta) \\
= & \iint:\left[: K_{1}\left(\xi, \xi^{\prime}, \eta\right): \xi^{\prime}:\left(\int \prod_{i=2}^{\ell}: K_{i}\left(\xi, \xi^{\prime}+\xi^{\prime \prime}, \eta\right): \xi^{\prime \prime} d \mu_{C}\left(\xi^{\prime \prime}\right)\right):_{\xi^{\prime}}\right] \cdot \eta_{\eta} \\
& \times f(\eta) d \mu_{C}\left(\xi^{\prime}\right) d \mu_{C}(\eta) \\
= & \iint: K_{1}\left(\xi, \xi^{\prime}, \eta\right): \xi^{\prime}, \eta:\left(\iint \prod_{i=2}^{\ell}: K_{i}\left(\xi, \xi^{\prime}+\xi^{\prime \prime}, \eta^{\prime}\right): \xi^{\prime \prime} d \mu_{C}\left(\xi^{\prime \prime}\right)\right): \xi_{\xi^{\prime}, \eta^{\prime}} \\
= & \iint\left(\eta+\eta^{\prime}\right): \eta d \mu_{C}\left(\eta^{\prime}\right) d \mu_{C}\left(\xi^{\prime}\right) d \mu_{C}(\eta) \\
& \times d \mu_{C}\left(\xi, \xi^{\prime}, \eta\right): \xi_{\xi^{\prime}, \eta}: \tilde{R}_{C}\left(K_{2}, \cdots, K_{\ell}(\eta)(f)\left(\xi, \xi^{\prime}, \eta\right): \xi^{\prime}, \eta\right.
\end{aligned}
$$

$\square$

Remark VII.2. Set

$$
\begin{aligned}
\hat{K}^{(i)}\left(\xi, \xi^{\prime} ; \xi^{\prime \prime}, \eta^{\prime}\right) & =K^{(i)}\left(\xi, \xi^{\prime}+\xi^{\prime \prime}, \eta^{\prime}\right), \\
\hat{f}\left(\xi, \xi^{\prime}\right) & =f\left(\xi+\xi^{\prime}\right) .
\end{aligned}
$$

Then the map $f \mapsto: \tilde{R}_{C}\left(K_{2}, \cdots, K_{\ell}\right)(f)$ : over the algebra $A^{\prime}$ agrees with the map $f \mapsto R_{C}\left(\hat{K}_{2}, \cdots, \hat{K}_{\ell}\right)(\hat{f})$ of (III.2) over the algebra $\tilde{A}$ of Grassmann functions in the variables $\xi^{\prime}, \eta$ with coefficients in $A^{\prime}$. Therefore we can use the results of $\S$ III to obtain estimates on $\tilde{R}_{C}$. 
Lemma VII.3. Let $K\left(\xi, \xi^{\prime}, \eta\right)$ be an even Grassmann function with $K\left(\xi, \xi^{\prime}, 0\right)=0$. Decompose

$$
K\left(\xi, \xi^{\prime}, \eta\right)=K^{\prime}\left(\xi, \xi^{\prime}, \eta\right)+K^{\prime \prime}\left(\xi, \xi^{\prime}, \eta\right)
$$

where $K^{\prime}$ has degree at most two in the variables $\xi^{\prime}, \eta$ and $K^{\prime \prime}$ has degree at least three in the variables $\xi^{\prime}, \eta$. Let each of the functions $K^{(1)}, \cdots, K^{(\ell)}$ be one of $K^{\prime}, K^{\prime \prime}$ or $K$, and assume that at least one of them is equal to $K^{\prime \prime}$. Let $f(\xi) \in \bigwedge_{A^{\prime}} V$, and set

$$
\frac{1}{\ell !} R_{C}\left(K^{(1)}, \cdots, K^{(\ell)}\right)(: f:)(\xi)=: f^{\prime}(\xi): .
$$

Then, if $\alpha \geq 2$,

$$
N_{\text {impr }}\left(f^{\prime} ; \alpha\right) \leq \frac{2^{5} J}{\ell \alpha^{\ell+5}} N(f ; 2 \alpha) N(K ; 2 \alpha)^{\ell} .
$$

Proof. We may assume that $K^{(1)}=K^{\prime \prime}$. Set

$$
g\left(\xi, \xi^{\prime}, \eta\right)=\tilde{R}_{C}\left(K^{(2)}, \cdots, K^{(\ell)}\right)(: f:)\left(\xi, \xi^{\prime}, \eta\right) .
$$

By Remark VII.2, in the algebra $\tilde{A}$,

$$
: g: \xi^{\prime}=R_{C}\left(\hat{K}^{(2)}, \cdots, \hat{K}^{(\ell)}\right)(: \hat{f}:)
$$

Therefore, by part (ii) of Prop. III.7 and Remark II.24,

$$
\begin{aligned}
\frac{1}{(\ell-1) !} N(g) & \leq \frac{1}{\alpha^{\ell-1}} N(\hat{f}) \prod_{i=2}^{\ell} N\left(\hat{K}^{(i)}\right) \\
& \leq \frac{1}{\alpha^{\ell-1}} N(f ; 2 \alpha) \prod_{i=2}^{\ell} N\left(K^{(i)} ; 2 \alpha\right) \\
& \leq \frac{1}{\alpha^{\ell-1}} N(f ; 2 \alpha) N(K ; 2 \alpha)^{\ell-1} .
\end{aligned}
$$

By Prop. VII.1,

$$
\left.f^{\prime}(\xi)=\frac{1}{\ell !} \iint: K^{\prime \prime}\left(\xi, \xi^{\prime}, \eta\right): \xi^{\prime}, \eta: g\left(\xi, \xi^{\prime}, \eta\right): \xi^{\prime}, \eta\right] \mu_{C}\left(\xi^{\prime}\right) d \mu_{C}(\eta)
$$

Proposition VI.4 implies that

$$
\begin{aligned}
N_{\mathrm{impr}}\left(f^{\prime} ; \alpha\right) & \leq \frac{27 J}{\ell ! \alpha^{6}} N\left(K^{\prime \prime} ; \alpha\right) N(g ; \alpha) \\
& \leq \frac{2^{5} J}{\ell \alpha^{\ell+5}} N(f ; 2 \alpha) N(K ; 2 \alpha)^{\ell}
\end{aligned}
$$

Proposition VII.4. Let $K\left(\xi, \xi^{\prime}, \eta\right)$ be an even Grassmann function. Decompose

$$
K\left(\xi, \xi^{\prime}, \eta\right)=K^{\prime}\left(\xi, \xi^{\prime}, \eta\right)+K^{\prime \prime}\left(\xi, \xi^{\prime}, \eta\right)
$$

where $K^{\prime}$ has degree at most two in the variables $\xi^{\prime}, \eta$ and $K^{\prime \prime}$ has degree at least three in the variables $\xi^{\prime}, \eta$. Let $f(\xi) \in \bigwedge_{A^{\prime}} V$, and set

$$
: g(\xi):=\mathcal{R}_{K, C}(: f:), \quad: g^{\prime}(\xi):=\mathcal{R}_{K^{\prime}, C}(: f:) .
$$

Then, if $\alpha \geq 2$ and $N(K ; 2 \alpha)_{\mathbf{0}} \leq \alpha$,

$$
N_{\text {impr }}\left(g-g^{\prime} ; \alpha\right) \leq \frac{2^{5} J}{\alpha^{6}} N(f ; 2 \alpha) \frac{N(K ; 2 \alpha)}{1-\frac{1}{\alpha} N(K ; 2 \alpha)} .
$$


Proof. By Remark III.6,

$$
g=\sum_{\ell=1}^{\infty} g_{\ell}, \quad g^{\prime}=\sum_{\ell=1}^{\infty} g_{\ell}^{\prime}
$$

where

$$
\begin{aligned}
& : g_{\ell}:=\frac{1}{\ell !} R_{C}(K, \cdots, K)(: f:), \\
& : g_{\ell}^{\prime}:=\frac{1}{\ell !} R_{C}\left(K^{\prime}, \cdots, K^{\prime}\right)(: f:)
\end{aligned}
$$

Since

$$
\begin{aligned}
R_{C}(K, \cdots, K)-R_{C}\left(K^{\prime}, \cdots, K^{\prime}\right)= & R_{C}\left(K-K^{\prime}, K, \cdots, K\right) \\
& +R_{C}\left(K^{\prime}, K-K^{\prime}, K, \cdots, K\right) \\
& +\cdots+R_{C}\left(K^{\prime}, \cdots, K-K^{\prime}\right) \\
= & R_{C}\left(K^{\prime \prime}, K, \cdots, K\right)+R_{C}\left(K^{\prime}, K^{\prime \prime}, K, \cdots, K\right) \\
& +\cdots+R_{C}\left(K^{\prime}, \cdots, K^{\prime}, K^{\prime \prime}\right)
\end{aligned}
$$

it follows from Lemma VII.3 that

$$
N_{\text {impr }}\left(g_{\ell}-g_{\ell}^{\prime}\right) \leq \frac{2^{5} J}{\alpha^{\ell+5}} N(f ; 2 \alpha) N(K ; 2 \alpha)^{\ell} .
$$

Therefore

$$
N_{\text {impr }}\left(g-g^{\prime}\right) \leq \sum_{\ell=1}^{\infty} N_{\text {impr }}\left(g_{\ell}-g_{\ell}^{\prime}\right) \leq \frac{2^{5} J}{\alpha^{6}} N(f ; 2 \alpha) \frac{N(K ; 2 \alpha)}{1-\frac{1}{\alpha} N(K ; 2 \alpha)} .
$$

Corollary VII.5. Under the hypotheses of Prop. VII.4, set

$$
: h:=\frac{1}{\mathbb{1}-\mathcal{R}_{K, C}}(: f:)-\frac{1}{1-\mathcal{R}_{K^{\prime}, C}}(: f:) .
$$

If $N(K ; 2 \alpha)_{\mathbf{0}}<\frac{\alpha}{6}$, then

$$
N_{\text {impr }}(h ; \alpha) \leq \frac{2^{5} J}{\alpha^{6}} \frac{N(K ; 2 \alpha)}{1-\frac{6}{\alpha} N(K ; 2 \alpha)} N(f ; 2 \alpha) .
$$

Proof. Since

$$
: h:=\left(\mathbb{1}-\mathcal{R}_{K, C}\right)^{-1}\left(\mathcal{R}_{K, C}-\mathcal{R}_{K^{\prime}, C}\right)\left(\mathbb{1}-\mathcal{R}_{K^{\prime}, C}\right)^{-1}(: f:)
$$

it follows from Cor. III.9, with $N_{\text {impr }}$ in place of $N$, and Prop. VII.4 that

$$
\begin{aligned}
N_{\text {impr }}(h) & \leq\left(1+\frac{2}{\alpha^{2}} \frac{N_{\text {impr }}(K)}{1-\frac{4}{\alpha^{2}} N_{\text {impr }}(K)}\right) N_{\text {impr }}\left(\left(\mathcal{R}_{K, C}-\mathcal{R}_{K^{\prime}, C}\right)\left(\mathbb{1}-\mathcal{R}_{K^{\prime}, C}\right)^{-1}(: f:)\right) \\
& \leq\left(1+\frac{2}{\alpha^{2}} \frac{N_{\text {impr }}(K)}{1-\frac{4}{\alpha^{2}} N_{\text {impr }}(K)}\right) \frac{2^{5} J}{\alpha^{6}} \frac{N(K ; 2 \alpha)}{1-\frac{1}{\alpha} N(K ; 2 \alpha)} N\left(\left(\mathbb{1}-\mathcal{R}_{K^{\prime}, C}\right)^{-1}(: f:) ; 2 \alpha\right) .
\end{aligned}
$$


By Def. VI.1 and Remark II.24, $N_{\text {impr }}(K) \leq N(K) \leq N(K ; 2 \alpha)$ so that Cor. III.9 implies

$$
\begin{aligned}
N_{\text {impr }}(h) \leq & \left(1+\frac{2}{\alpha^{2}} \frac{N(K ; 2 \alpha)}{1-\frac{4}{\alpha^{2}} N(K ; 2 \alpha)}\right) \frac{2^{5} J}{\alpha^{6}} \frac{N(K ; 2 \alpha)}{1-\frac{1}{\alpha} N(K ; 2 \alpha)} \\
& \times\left(1+\frac{1}{2 \alpha^{2}} \frac{N\left(K^{\prime} ; 2 \alpha\right)}{1-\frac{1}{\alpha^{2}} N\left(K^{\prime} ; 2 \alpha\right)}\right) N(f ; 2 \alpha) \\
\leq & \frac{2^{5} J}{\alpha^{6}}\left(\frac{1-\frac{2}{\alpha^{2}} N(K ; 2 \alpha)}{1-\frac{4}{\alpha^{2}} N(K ; 2 \alpha)}\right) \frac{N(K ; 2 \alpha)}{1-\frac{1}{\alpha} N(K ; 2 \alpha)}\left(\frac{1-\frac{1}{\alpha^{2}} N(K ; 2 \alpha)}{1-\frac{1}{\alpha^{2}} N(K ; 2 \alpha)}\right) N(f ; 2 \alpha) \\
\leq & \frac{2^{5} J}{\alpha^{6}} \frac{1}{1-\frac{4}{\alpha^{2}} N(K ; 2 \alpha)} \frac{N(K ; 2 \alpha)}{1-\frac{1}{\alpha} N(K ; 2 \alpha)} \frac{1}{1-\frac{1}{\alpha^{2}} N(K ; 2 \alpha)} N(f ; 2 \alpha) \\
\leq & \frac{2^{5} J}{\alpha^{6}} \frac{N(K ; 2 \alpha)}{\left[1-\frac{2}{\alpha} N(K ; 2 \alpha)\right]^{3}} N(f ; 2 \alpha) \\
\leq & \frac{2^{5} J}{\alpha^{6}} \frac{N(K ; 2 \alpha)}{1-\frac{6}{\alpha} N(K ; 2 \alpha)} N(f ; 2 \alpha)
\end{aligned}
$$

since, for $X \in \mathcal{N}_{d}$,

$$
\frac{1}{[1-X]^{3}}=\sum_{r=0}^{\infty}\left(\begin{array}{c}
-3 \\
r
\end{array}\right)(-X)^{r}=\sum_{r=0}^{\infty} \frac{3 \cdot 4 \cdot 5 \cdots(3+r-1)}{1 \cdot 2 \cdot 3 \cdots r} X^{r} \leq \sum_{r=0}^{\infty}(3 X)^{r} .=\frac{1}{1-3 X} .
$$

Proposition VII.4 exploits overlapping loops that are created by one application of the operator $\mathcal{R}_{K, C}$. There are additional overlapping loops created by the composite operator $\mathcal{R}_{K, C} \circ \mathcal{R}_{K, C}$ which we shall exploit now.

Lemma VII.6. Let $B\left(\eta^{\prime}, \eta^{\prime \prime}\right) \in A^{\prime}[1,1]$ and let $H\left(\xi, \xi^{\prime}, \eta\right), K\left(\xi, \xi^{\prime}, \eta\right)$ be even Grassmann functions that vanish for $\eta=0$. Assume that $H$ or $K$ has degree at least two in the variables $\xi^{\prime}, \eta$. Let $f(\xi) \in \bigwedge_{A^{\prime}} V$ and set

$$
\begin{aligned}
& g(\xi)=\int:\left[\iiint B\left(\eta^{\prime}, \eta^{\prime \prime}\right): H\left(\xi+\eta^{\prime}, \xi^{\prime}, \eta\right): \eta^{\prime}, \xi^{\prime}: K\left(\xi+\eta^{\prime \prime}, \xi^{\prime}, \eta\right): \eta^{\prime \prime}, \xi^{\prime}\right. \\
& \left.\times d \mu_{C}\left(\eta^{\prime}, \eta^{\prime \prime}, \xi^{\prime}\right)\right]: \eta_{\eta}: f(\eta):{ }_{\eta} d \mu_{C}(\eta) .
\end{aligned}
$$

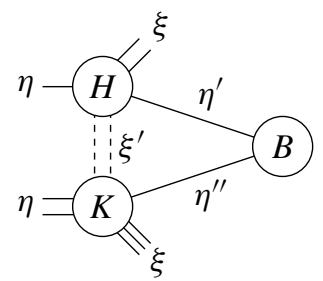

Then, if $\alpha \geq 2$,

$$
N_{\mathrm{impr}}(g ; \alpha) \leq \frac{2^{5} J}{\alpha^{10}} N(f ; 2 \alpha) N(H ; 2 \alpha) N(K ; 2 \alpha) N(B ; \alpha) .
$$


Proof. We may assume that $K$ has degree at least two in the variables $\xi^{\prime}, \eta$. Set

$$
h\left(\xi, \eta, \xi^{\prime}, \eta^{\prime \prime}\right)=\iint B\left(\eta^{\prime}, \eta^{\prime \prime}\right): H\left(\xi+\eta^{\prime}, \xi^{\prime}, \zeta\right):_{\eta^{\prime}, \xi, \zeta}: f(\eta+\zeta):_{\zeta} d \mu_{C}\left(\eta^{\prime}\right) d \mu_{C}(\zeta)
$$

By Lemma A.5, applied to the variable $\eta$,

$$
\begin{aligned}
g(\xi)= & \int B\left(\eta^{\prime}, \eta^{\prime \prime}\right): H\left(\xi+\eta^{\prime}, \xi^{\prime}, \zeta\right):_{\eta^{\prime}, \xi^{\prime}, \zeta}: K\left(\xi+\eta^{\prime \prime}, \xi^{\prime}, \eta\right): \eta^{\prime \prime}, \xi^{\prime}, \eta \\
& : f(\eta+\zeta):_{\eta, \zeta} d \mu_{C}\left(\eta^{\prime}, \eta^{\prime \prime}, \xi^{\prime}, \eta, \zeta\right) \\
= & \int: h\left(\xi, \eta, \xi^{\prime}, \eta^{\prime \prime}\right): \xi^{\prime}, \eta \\
& : K\left(\xi+\eta^{\prime \prime}, \xi^{\prime}, \eta\right):_{\eta^{\prime \prime}, \xi^{\prime}, \eta} d \mu_{C}\left(\eta, \eta^{\prime \prime}, \xi^{\prime}\right) .
\end{aligned}
$$

Since $B$ is of degree one in $\eta^{\prime}$ and $H$ is of degree at least one in $\zeta$, iterated application of Prop. II.33 and Remark II.24 yields

$$
N(h) \leq \frac{1}{\alpha^{4}} N(B) N(H ; 2 \alpha) N(f ; 2 \alpha) .
$$

Since $h$ is of degree one in $\eta^{\prime \prime}$, only the part of $K$ that is of degree at least three in the variables $\eta^{\prime \prime}, \xi^{\prime}, \eta$ can contribute. Hence Prop. VI.4 implies that

$$
\begin{aligned}
N_{\text {impr }}(g) & \leq \frac{27 J}{\alpha^{6}} N(h) N(K ; 2 \alpha) \\
& \leq \frac{2^{5} J}{\alpha^{10}} N(B) N(H ; 2 \alpha) N(f ; 2 \alpha) N(K ; 2 \alpha) .
\end{aligned}
$$

$\square$

Proposition VII.7. Let $K\left(\xi, \xi^{\prime}, \eta\right)$ be a Grassmann function of degree at most two in the variables $\xi^{\prime}, \eta$ and of degree at least one in $\eta$. Write

$$
K\left(\xi, \xi^{\prime}, \eta\right)=\sum_{n_{1}, n_{2}, n_{3}} K_{n_{1}, n_{2}, n_{3}}\left(\xi, \xi^{\prime}, \eta\right) \quad \text { with } \quad K_{n_{1}, n_{2}, n_{3}} \in A^{\prime}\left[n_{1}, n_{2}, n_{3}\right] .
$$

Also set $K_{\cdot, n_{2}, n_{3}}=\sum_{n_{1}} K_{n_{1}, n_{2}, n_{3}}$. Furthermore let $T(\eta) \in A^{\prime}[2]$ and write

$$
T\left(\eta+\eta^{\prime}\right)=T(\eta)+T\left(\eta^{\prime}\right)+T_{\text {mix }}\left(\eta, \eta^{\prime}\right)
$$

with $T_{\text {mix }} \in A^{\prime}[1,1]$. Set

$$
\begin{aligned}
& \tilde{K}\left(\xi, \xi^{\prime}, \eta\right)=\int: T\left(\eta^{\prime}\right): \eta^{\prime}: K\left(\xi+\eta^{\prime}, \xi^{\prime}, \eta\right): \eta^{\prime} d \mu_{C}\left(\eta^{\prime}\right) \\
& \hat{K}\left(\xi, \xi^{\prime}, \eta\right)=\int T_{\operatorname{mix}}\left(\eta^{\prime}, \eta^{\prime \prime}\right): K_{\cdot, 0,1}\left(\xi+\eta^{\prime}, \xi^{\prime}, \eta\right): \eta^{\prime}: K_{\cdot, 0,1}\left(\xi+\eta^{\prime \prime}, \xi^{\prime}, \eta\right): \eta^{\prime \prime} d \mu_{C}\left(\eta^{\prime}, \eta^{\prime \prime}\right)
\end{aligned}
$$

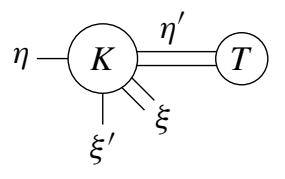

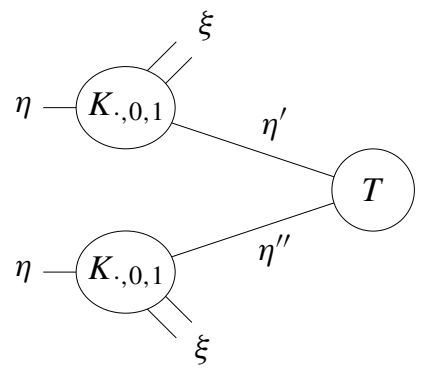


Observe that $\hat{K}$ is independent of $\xi^{\prime}$. Finally let $f(\xi) \in \bigwedge_{A^{\prime}} V$ and set

$$
\begin{aligned}
f^{\prime}(\eta) & =\tilde{R}_{C}(T) R_{C}(K, K)(: f:), \\
: \tilde{f}(\xi): \xi & =2 R_{C}(\tilde{K}, K)(: f:), \\
: \hat{f}(\xi): \xi & =R_{C}(\hat{K})(: f:) .
\end{aligned}
$$

Then

$$
N_{\text {impr }}\left(f^{\prime}-(\tilde{f}+\hat{f}) ; \alpha\right) \leq \frac{2^{6} J}{\alpha^{10}} N(f ; 2 \alpha) N(K ; 2 \alpha)^{2} N(T ; 2 \alpha) .
$$

Proof. By definition

$$
f^{\prime}(\eta)=\int: T\left(\eta^{\prime}\right): \eta^{\prime} R_{C}(K, K)(: f:)\left(\eta+\eta^{\prime}\right) d \mu_{C}\left(\eta^{\prime}\right) .
$$

Consequently, by part (ii) of Prop. A.2 and Lemma A.7, in the variable $\eta^{\prime}$,

$$
\begin{aligned}
& f^{\prime}(\xi)=\int: T\left(\eta^{\prime}\right): \eta^{\prime} R_{C}(K, K)(: f:)\left(\xi+\eta^{\prime}\right) d \mu_{C}\left(\eta^{\prime}\right) \\
& =\int: T\left(\eta^{\prime}\right): \eta^{\prime} \cdot\left[:\left(: K\left(\xi+\eta^{\prime}, \xi^{\prime}, \eta\right): \xi^{\prime}: K\left(\xi+\eta^{\prime}, \xi^{\prime}, \eta\right): \xi^{\prime}\right): \eta: f(\eta): \eta\right]: \eta^{\prime} \\
& \times d \mu_{C}\left(\xi^{\prime}, \eta, \eta^{\prime}\right) \\
& =2 \int:\left[:\left(\int: T\left(\eta^{\prime}\right): \eta_{\eta^{\prime}}: K\left(\xi+\eta^{\prime}, \xi^{\prime}, \eta\right): \eta^{\prime} d \mu_{C}\left(\eta^{\prime}\right)\right):_{\xi^{\prime}}: K\left(\xi, \xi^{\prime}, \eta\right): \xi_{\xi^{\prime}}\right] \cdot{ }_{\eta} \\
& : f(\eta):_{\eta} d \mu_{C}\left(\xi^{\prime}, \eta\right) \\
& +\int:\left[\int T_{\text {mix }}\left(\eta^{\prime}, \eta^{\prime \prime}\right): K\left(\xi+\eta^{\prime}, \xi^{\prime}, \eta\right): \xi^{\prime}, \eta^{\prime}: K\left(\xi+\eta^{\prime \prime}, \xi^{\prime}, \eta\right): \xi^{\prime}, \eta^{\prime \prime}\right. \\
& \left.\times d \mu_{C}\left(\xi^{\prime}, \eta^{\prime}, \eta^{\prime \prime}\right)\right]_{\eta_{\eta}}: f(\eta): \eta d \mu_{C}(\eta) \\
& =2 \int:\left[: \tilde{K}\left(\xi, \xi^{\prime}, \eta\right): \xi^{\prime}: K\left(\xi, \xi^{\prime}, \eta\right): \xi^{\prime}\right]: \eta f(\eta): \eta d \mu_{C}\left(\xi^{\prime}, \eta\right) \\
& +\int: \hat{K}\left(\xi, \xi^{\prime}, \eta\right): \xi^{\prime}, \eta: f(\eta): \eta d \mu_{C}\left(\xi^{\prime}, \eta\right) \\
& +\int:\left[\int T _ { \text { mix } } ( \eta ^ { \prime } , \eta ^ { \prime \prime } ) \left(: K\left(\xi+\eta^{\prime}, \xi^{\prime}, \eta\right): \xi^{\prime}, \eta^{\prime}: K\left(\xi+\eta^{\prime \prime}, \xi^{\prime}, \eta\right): \xi^{\prime}, \eta^{\prime \prime}\right.\right. \\
& \left.-: K_{, 0,1}\left(\xi+\eta^{\prime}, \xi^{\prime}, \eta\right): \xi^{\prime}, \eta^{\prime}: K_{\cdot, 0,1}\left(\xi+\eta^{\prime \prime}, \xi^{\prime}, \eta\right): \xi^{\prime}, \eta^{\prime \prime}\right) \\
& \left.\times d \mu_{C}\left(\xi^{\prime}, \eta^{\prime}, \eta^{\prime \prime}\right)\right]: \eta_{\eta}: f(\eta): \eta d \mu_{C}(\eta) \\
& =\tilde{f}(\xi)+\hat{f}(\xi)+g^{\prime}(\xi)+g^{\prime \prime}(\xi)
\end{aligned}
$$

with

$$
\begin{aligned}
g^{\prime}(\xi)= & \int:\left[\int T_{\operatorname{mix}}\left(\eta^{\prime}, \eta^{\prime \prime}\right): H\left(\xi+\eta^{\prime}, \xi^{\prime}, \eta\right): \xi^{\prime}, \eta^{\prime}: K\left(\xi+\eta^{\prime \prime}, \xi^{\prime}, \eta\right): \xi^{\prime}, \eta^{\prime \prime}\right. \\
& \left.\times d \mu_{C}\left(\xi^{\prime}, \eta^{\prime}, \eta^{\prime \prime}\right)\right]: \eta_{\eta}: f(\eta): \eta d \mu_{C}(\eta), \\
g^{\prime \prime}(\xi)= & \int:\left[\int T_{\operatorname{mix}}\left(\eta^{\prime}, \eta^{\prime \prime}\right): K_{\cdot, 0,1}\left(\xi+\eta^{\prime}, \xi^{\prime}, \eta\right): \xi^{\prime}, \eta^{\prime}\right. \\
& : H\left(\xi+\eta^{\prime \prime}, \xi^{\prime}, \eta\right): \xi^{\prime}, \eta^{\prime \prime} \\
& \left.\times d \mu_{C}\left(\xi^{\prime}, \eta^{\prime}, \eta^{\prime \prime}\right)\right]:_{\eta}: f(\eta): \eta d \mu_{C}(\eta),
\end{aligned}
$$


where $H=K-K_{\cdot, 0,1}$. In the last equality, we used the fact that $K_{\cdot, 0,1}\left(\xi+\eta^{\prime}, \xi^{\prime}, \eta\right)$ and hence $\hat{K}$ is independent of $\xi^{\prime}$, so that we are free to drop the Wick ordering with respect to $\xi^{\prime}$ in the expression yielding $\hat{f}(\xi)$. By Lemma VII.6 and the observations that

$$
\begin{aligned}
& N\left(K_{\cdot, 0,1} ; 2 \alpha\right), N\left(K-K_{\cdot, 0,1} ; 2 \alpha\right) \leq N(K ; 2 \alpha), \\
& N\left(T_{\text {mix }} ; \alpha\right) \leq N\left(T\left(\eta+\eta^{\prime}\right) ; \alpha\right) \leq N(T ; 2 \alpha),
\end{aligned}
$$

we have

$$
N_{\mathrm{impr}}\left(f^{\prime}-\tilde{f}-\hat{f}\right)=N_{\mathrm{impr}}\left(g^{\prime}+g^{\prime \prime}\right) \leq \frac{2^{6} J}{\alpha^{10}} N(f ; 2 \alpha) N(K ; 2 \alpha)^{2} N(T ; 2 \alpha) .
$$

VII.2. Tails. In this subsection let $K\left(\psi ; \xi, \xi^{\prime}, \eta\right)$ be an even Grassmann function with coefficients in $A$ that has degree at least four in the variables $\psi, \xi, \xi^{\prime}, \eta$ and degree at least one in the variable $\eta$. We always write

$$
K=\sum_{n_{0}, \cdots, n_{3}} K_{n_{0}, n_{1}, n_{2}, n_{3}} \quad \text { with } \quad K_{n_{0}, n_{1}, n_{2}, n_{3}} \in A\left[n_{0}, n_{1}, n_{2}, n_{3}\right] .
$$

For $f(\psi ; \xi): \in \bigwedge_{A}\left(V^{\prime} \oplus V\right)$ we are interested in the two and four legged contributions to $\frac{1}{\mathbb{1}-\mathcal{R}_{K, C}}(: f: \xi)$. Therefore we make the following

Definition VII.8. The projection

$$
\begin{aligned}
P: \bigwedge_{A}\left(V^{\prime} \oplus V\right) & \longrightarrow \bigwedge_{A} V^{\prime} \\
: f(\psi ; \xi): \xi & \longmapsto f_{4,0}(\psi, 0)+f_{2,0}(\psi, 0),
\end{aligned}
$$

where $f(\psi ; \xi)=\sum_{n_{0}, n_{1}} f_{n_{0}, n_{1}}(\psi ; \xi)$ with $f_{n_{0}, n_{1}} \in A\left[n_{0}, n_{1}\right]$.

Definition VII.9. (i) An n-legged tail is a Grassmann function $T(\psi ; \eta) \in$ $\bigoplus_{d \geq 2} A[d, n]$. We say that an n-legged tail $T$ has at least $d$ external legs if $T \in \bigoplus_{d^{\prime} \geq d} A\left[d^{\prime}, n\right]$.

(ii) If $T$ is a two-legged tail we define the two-legged tail $T \circ K$ by

$$
\begin{gathered}
(T \circ K)(\psi ; \eta)=\int: T\left(\psi ; \eta^{\prime}\right): \eta^{\prime}: K_{0,2,0,2}\left(\psi ; \eta^{\prime}, \xi^{\prime}, \eta\right): \eta^{\prime} d \mu_{C}\left(\eta^{\prime}\right) \\
\eta=T=\eta^{\prime} T \psi
\end{gathered}
$$

Observe that $T \circ K$ depends only on the part of $K$ that has degree at most two in the variables $\xi^{\prime}, \eta$.

Remark VII.10. A two-legged tail with two external legs is an end in the sense of Def. VI.5.i. If $K\left(\psi ; \xi, \xi^{\prime}, \eta\right)=U\left(\psi ; \xi+\xi^{\prime}+\eta\right)-U\left(\psi ; \xi+\xi^{\prime}\right)$ for some even Grassmann function $U(\psi ; \xi)$ then $T \circ K$ agrees with $T \circ \operatorname{Rung}(U)$ of Def. VI.5.iii. 
Lemma VII.11. Assume that $K$ has degree at most two in the variables $\xi^{\prime}, \eta$. Let $T$ be a two-legged tail. Then there exists a one-legged tail $t_{1}$ with at least three external legs and a two-legged tail $t_{2}$ with at least three external legs such that for any $f(\psi ; \xi) \in \bigwedge_{A}\left(V^{\prime} \oplus V\right)$ the following holds:

Set

$$
\begin{aligned}
f^{\prime}(\psi)= & P\left[R_{C}(T) \mathcal{R}_{K, C}(: f:)-R_{C}(T \circ K)(: f:)-R_{C}\left(T \circ K, K_{2,0,0,2}\right)(: f:)\right. \\
& \left.-R_{C}\left(t_{1}+t_{2}\right)(: f:)\right]
\end{aligned}
$$

where $: f:$ is shorthand for $: f: \xi$. Then

$$
N_{\text {impr }}\left(f^{\prime} ; \alpha\right) \leq \frac{2^{6} J}{\alpha^{10}} N(f ; 2 \alpha) N(K ; 2 \alpha)^{2} N(T ; 2 \alpha) .
$$

If $T$ has at least three external legs then

$$
P R_{C}(T) \mathcal{R}_{K, C}(: f:)=P R_{C}(T \circ K)(: f:)+P R_{C}\left(t_{1}+t_{2}\right)(: f:) .
$$

Proof. By assumption, $K\left(\psi ; \xi, \xi^{\prime}, \eta\right)$ is of degree at least two in $\psi$, $\xi$, so $R_{C}(K, \cdots, K)$ (:f:) (with $\ell K$ 's) is of degree at least $2 \ell$ in $\psi, \xi$. Since $T$ is two-legged, $R_{C}(T)$ $R_{C}(K, \cdots, K)(: f:)$ is of degree at least $2 \ell-2+2=2 \ell$ (with the last +2 coming from the $d \geq 2$ external legs of $T$ ) in $\psi$ and is independent of $\xi$. So, by Remark III.6,

$$
P\left[R_{C}(T) \mathcal{R}_{K, C}(: f:)\right]=P\left[R_{C}(T) R_{C}(K)(: f:)\right]+\frac{1}{2} P\left[R_{C}(T) R_{C}(K, K)(: f:)\right] .
$$

Set

$$
\begin{aligned}
t_{11}(\psi ; \eta)= & \int: T\left(\psi ; \eta^{\prime}\right):_{\eta^{\prime}}: K_{\cdot, 2,0,1}\left(\psi ; \eta^{\prime}, \xi^{\prime}, \eta\right): \eta^{\prime} \\
t_{21}(\psi ; \eta)= & \int: T\left(\psi ; \mu_{C}\left(\eta^{\prime}\right)::_{\eta^{\prime}}:\left(K_{\cdot, 2,0,2}\left(\psi ; \eta^{\prime}, \xi^{\prime}, \eta\right)\right.\right. \\
& \left.-K_{0,2,0,2}\left(\psi ; \eta^{\prime}, \xi^{\prime}, \eta\right)\right):_{\eta^{\prime}} d \mu_{C}\left(\eta^{\prime}\right) .
\end{aligned}
$$

Since $K$ has degree at least four overall and $T$ has degree at least two in $\psi, t_{11}$ is a one-legged tail and $t_{21}$ is a two-legged tail, both having at least three external legs. As $K$ has degree at most two in the variables $\xi^{\prime}, \eta$ and degree at least one in $\eta$ and $T$ has degree two in $\eta$ and the definition of $R_{C}(K)$ Wick orders $K$ with respect to $\xi^{\prime}$,

$$
\begin{aligned}
P\left[R_{C}(T) R_{C}(K)(: f:)\right] & =P\left[R_{C}(T) R_{C}\left(K_{\cdot, 2,0,1}\right)(: f:)\right]+P\left[R_{C}(T) R_{C}\left(K_{\cdot, 2,0,2}\right)(: f:)\right] \\
& =P\left[R_{C}\left(t_{11}+t_{21}\right)(: f:)\right]+P\left[R_{C}(T \circ K)(: f:)\right] .
\end{aligned}
$$

Define the projection

$$
\begin{aligned}
P^{\prime}: \bigwedge_{A}\left(V^{\prime} \oplus V\right) & \longrightarrow \bigwedge_{A} V^{\prime} \\
. f(\psi ; \xi) & \longmapsto f_{4,0}(\psi, 0)+f_{2,0}(\psi, 0) .
\end{aligned}
$$

As $P$ and $P^{\prime}$ only differ by Wick ordering in the $\xi$-argument,

$$
\begin{aligned}
P\left[R_{C}(T) R_{C}(K, K)(: f:)\right] & =P^{\prime}\left[R_{C}(T) R_{C}(K, K)(: f:)\right] \\
& =P^{\prime}\left[\tilde{R}_{C}(T) R_{C}(K, K)(: f:)(\psi ; 0,0, \xi)\right],
\end{aligned}
$$


so that we can apply Prop. VII.7. Modulo a term whose improved norm $N_{\text {impr }}$ is bounded by $\frac{2^{6} J}{\alpha^{10}} N(f ; 2 \alpha) N(K ; 2 \alpha)^{2} N(T ; 2 \alpha)$,

$$
P^{\prime}\left[\tilde{R}_{C}(T) R_{C}(K, K)(: f:)(\psi ; 0,0, \xi)\right]=2 P\left[R_{C}(\tilde{K}, K)(: f:)\right]+P\left[R_{C}(\hat{K})(: f:)\right]
$$

with

$$
\begin{aligned}
\tilde{K}\left(\psi ; \xi, \xi^{\prime}, \eta\right)= & \int: T\left(\psi ; \eta^{\prime}\right): \eta^{\prime} \\
\hat{K}\left(\psi ; \xi\left(\psi ; \eta^{\prime}, \xi^{\prime}, \eta\right): \eta^{\prime}\right. & d \mu_{C}\left(\eta^{\prime}\right), \\
& : K_{\cdot, 1,0,1}\left(\psi ; \eta^{\prime \prime}, \xi^{\prime}, \eta\right): \eta^{\prime \prime} d \mu_{C}\left(\eta^{\prime}, \eta^{\prime \prime}\right) .
\end{aligned}
$$

Here we have used the projection $P$ to set $\xi=0$ and we used that $T_{\text {mix }}\left(\psi ; \eta^{\prime}, \eta^{\prime \prime}\right)$ is of degree one in $\eta^{\prime}$ and in $\eta^{\prime \prime}$. Again, since $K$ has degree at least four overall and $T$ is of degree at least two in $\psi$, the tail $\hat{K}$ has at least six external legs, so that $P\left[R_{C}(\hat{K})(: f:)\right]=$ 0 . Finally, since

- $P$ sets the $\xi$ 's in the argument $K$ of $P\left[R_{C}(\tilde{K}, K)(: f:)\right]$ to zero.

- $\tilde{K}$ is of degree at least two in $\psi$.

- $K_{\cdot, 0,0,1}$ is of degree at least three in $\psi$.

- $K$ is of degree at most two in $\xi^{\prime}, \eta$ and degree at least one in $\eta$,

we have

$$
\begin{aligned}
P\left[R_{C}(\tilde{K}, K)(: f:)\right] & =P\left[R_{C}\left(\tilde{K}_{\cdot, 0, \cdot, \cdot}, K_{\cdot, 0,0,2}\right)(: f:)\right]+P\left[R_{C}\left(\tilde{K}_{\cdot, 0, \cdot, \cdot}, K_{\cdot, 0,1,1}\right)(: f:)\right] \\
& =P\left[R_{C}\left(\tilde{K}_{\cdot, 0,0, .,} K_{\cdot, 0,0,2}\right)(: f:)\right]+P\left[R_{C}\left(\tilde{K}_{\cdot, 0,1, \cdot,} K_{\cdot, 0,1,1}\right)(: f:)\right],
\end{aligned}
$$

and since

- $K$ is of degree at least four overall and $\tilde{K}$ is of degree at least two in $\psi$, we have

$$
\begin{aligned}
P\left[R_{C}(\tilde{K}, K)(: f:)\right]= & P\left[R_{C}\left(\tilde{K}_{2,0,0, .}, K_{2,0,0,2}\right)(: f:)\right] \\
& +P\left[R_{C}\left(\tilde{K}_{2,0,1, .}, K_{2,0,1,1}\right)(: f:)\right] \\
= & P\left[R_{C}\left(\tilde{K}_{2,0,0,2}, K_{2,0,0,2}\right)(: f:)\right] \\
& +P\left[R_{C}\left(\tilde{K}_{2,0,1,1}, K_{2,0,1,1}\right)(: f:)\right] \\
= & P\left[R_{C}\left(T \circ K, K_{2,0,0,2}\right)(: f:)\right]+P\left[R_{C}\left(t_{22}\right)(: f:)\right],
\end{aligned}
$$

where

$$
t_{22}(\psi ; \eta)=\int \tilde{K}_{2,0,1,1}\left(\psi ; \xi, \xi^{\prime}, \eta\right) K_{2,0,1,1}\left(\psi ; \xi, \xi^{\prime}, \eta\right) d \mu_{C}\left(\xi^{\prime}\right)
$$

is a two-legged tail with at least four external legs.

Setting $t_{1}=t_{11}$ and $t_{2}=t_{21}+2 t_{22}$ yields the main result. If $T$ has at least three external legs, then $R_{C}(T) R_{C}(K, \cdots, K)(: f:)$ (with $\ell K$ 's) is of degree at least $2 \ell-2+3=$ $2 \ell+1$, so

$$
\begin{aligned}
P\left[R_{C}(T) \mathcal{R}_{K, C}(: f:)\right] & =P\left[R_{C}(T) R_{C}(K)(: f:)\right] \\
& =P\left[R_{C}\left(t_{11}+t_{21}\right)(: f:)\right]+P\left[R_{C}(T \circ K)(: f:)\right] .
\end{aligned}
$$


Lemma VII.12. Assume that $K$ has degree at most two in the variables $\xi^{\prime}, \eta$. Let $T_{1}, T_{2}$ be two-legged tails. Then there exists a one-legged tail $t_{1}$ and a two-legged tail t $t_{2}$, each with at least four external legs, such that for any $f(\psi ; \xi) \in \bigwedge_{A}\left(V^{\prime} \oplus V\right)$ the following holds:

Set

$$
f^{\prime}(\psi)=P\left[R_{C}\left(T_{1}, T_{2}\right) \mathcal{R}_{K, C}(: f:)-R_{C}\left(T_{1} \circ K, T_{2} \circ K\right)(: f:)-R_{C}\left(t_{1}+t_{2}\right)(: f:)\right] .
$$

Then, if $\alpha \geq 2$,

$$
N_{\text {impr }}\left(f^{\prime} ; \alpha\right) \leq \frac{2^{6} J}{\alpha^{12}} N(f ; 2 \alpha) N(K ; 2 \alpha)^{2} N\left(T_{1} ; 2 \alpha\right) N\left(T_{2} ; 2 \alpha\right) .
$$

Proof. Again, since $K$ has degree at most two in the variables $\xi^{\prime}, \eta$ and degree at least four in all variables,

$$
\begin{aligned}
P\left[R_{C}\left(T_{1}, T_{2}\right) \mathcal{R}_{K, C}(: f:)\right]= & P\left[R_{C}\left(T_{1}, T_{2}\right) R_{C}(K)(: f:)\right] \\
& +\frac{1}{2} P\left[R_{C}\left(T_{1}, T_{2}\right) R_{C}(K, K)(: f:)\right]
\end{aligned}
$$

and

$$
\begin{aligned}
P\left[R_{C}\left(T_{1}, T_{2}\right) R_{C}(K)(: f:)\right] & =P\left[R _ { C } ( T _ { 1 } , T _ { 2 } ) R _ { C } \left(K_{0,4,0, .)(: f:)]}\right.\right. \\
& =P\left[R_{C}\left(t_{11}+t_{21}\right)(: f:)\right]
\end{aligned}
$$

where

$$
\begin{aligned}
& t_{11}(\psi ; \eta)=\int: T_{1}\left(\psi ; \eta^{\prime}\right) T_{2}\left(\psi ; \eta^{\prime}\right): \eta^{\prime} K_{0,4,0,1}\left(\psi ; \eta^{\prime}, \xi^{\prime}, \eta\right) d \mu_{C}\left(\eta^{\prime}\right), \\
& t_{21}(\psi ; \eta)=\int: T_{1}\left(\psi ; \eta^{\prime}\right) T_{2}\left(\psi ; \eta^{\prime}\right): \eta_{\eta^{\prime}} K_{0,4,0,2}\left(\psi ; \eta^{\prime}, \xi^{\prime}, \eta\right) d \mu_{C}\left(\eta^{\prime}\right)
\end{aligned}
$$

are one- resp. two-legged tails with at least four external legs. Similarly

$$
\begin{aligned}
P\left[R_{C}\left(T_{1}, T_{2}\right) R_{C}(K, K)(: f:)\right]= & P\left[R_{C}\left(T_{1}, T_{2}\right) R_{C}\left(K_{0,2,0,2}, K_{0,2,0,2}\right)(: f:)\right] \\
& +P\left[R_{C}\left(T_{1}, T_{2}\right) R_{C}\left(K_{0,2,1,1}, K_{0,2,1,1}\right)(: f:)\right] .
\end{aligned}
$$

The second term is $P R_{C}\left(t_{22}\right)(: f:)$, where

$$
\begin{aligned}
t_{22}(\psi ; \eta)= & \int: T_{1}\left(\psi ; \eta^{\prime}\right) T_{2}\left(\psi ; \eta^{\prime}\right): \eta_{\eta^{\prime}}: K_{0,2,1,1}\left(\psi ; \eta^{\prime}, \xi^{\prime}, \eta\right) \\
& \times K_{0,2,1,1}\left(\psi ; \eta^{\prime}, \xi^{\prime}, \eta\right): \eta^{\prime} d \mu_{C}\left(\eta^{\prime}\right)
\end{aligned}
$$

is a two-legged tail with at least four external legs. To deal with the first term we use Prop. VII.1 to see that

$$
R_{C}\left(T_{1}, T_{2}\right) R_{C}\left(K_{0,2,0,2}, K_{0,2,0,2}\right)(: f:)=\int: T_{1}\left(\psi, \eta^{\prime}\right): \eta^{\prime}: g\left(\psi ; \eta^{\prime}\right): \eta^{\prime} d \mu_{C}\left(\eta^{\prime}\right),
$$

where

$$
g(\psi ; \eta)=\tilde{R}_{C}\left(T_{2}\right) R_{C}\left(K_{0,2,0,2}, K_{0,2,0,2}\right)(: f:) .
$$


By Prop. VII.7, $: g(\psi ; \xi): \xi$ is - modulo terms whose improved norm can be bounded by $\frac{2^{6} J}{\alpha^{10}} N(f ; 2 \alpha) N(K ; 2 \alpha)^{2} N\left(T_{2} ; 2 \alpha\right)$ - equal to $2 R_{C}\left(K_{0,2,0,2}, T_{2} \circ K\right)(: f:)$. Therefore by Prop. II.33, modulo terms whose improved norm can be bounded by $\frac{2^{6} J}{\alpha^{12}} N(f ; 2 \alpha)$ $N(K ; 2 \alpha)^{2} N_{\text {impr }}\left(T_{1} ; \alpha\right) N\left(T_{2} ; 2 \alpha\right)$,

$$
\begin{aligned}
\frac{1}{2} P & {\left[R_{C}\left(T_{1}, T_{2}\right) R_{C}\left(K_{0,2,0,2}, K_{0,2,0,2}\right)(: f:)\right] } \\
& =P \int: T_{1}\left(\psi, \eta^{\prime}\right): \eta^{\prime} R_{C}\left(K_{0,2,0,2}, T_{2} \circ K\right)(: f:)\left(\psi ; \eta^{\prime}\right) d \mu_{C}\left(\eta^{\prime}\right) \\
& =P\left[R_{C}\left(T_{1} \circ K, T_{2} \circ K\right)(: f:)\right] .
\end{aligned}
$$

Since $N_{\text {impr }}\left(T_{1} ; \alpha\right) \leq N\left(T_{1} ; \alpha\right) \leq N\left(T_{1} ; 2 \alpha\right)$, the lemma follows.

Lemma VII.13. Assume that $K$ has degree at most two in the variables $\xi^{\prime}, \eta$. Let $T$ be a one-legged tail with at least three external legs. Then there is a two-legged tail t $t_{2}$ with at least four external legs such that for all $f(\psi ; \xi) \in \bigwedge_{A}\left(V^{\prime} \oplus V\right)$,

$$
P\left[R_{C}(T) \mathcal{R}_{K, C}(: f:)\right]=P\left[R_{C}\left(t_{2}\right)(: f:)\right] .
$$

Proof. Since $T$ is one-legged and $K$ has degree at least four

$$
\begin{aligned}
P R_{C}(T) \mathcal{R}_{K, C} & =P R_{C}(T) R_{C}(K) \\
& =P R_{C}(T) R_{C}\left(\sum_{d \geq 2} K_{d, 1,0,1}+\sum_{d \geq 1} K_{d, 1,0,2}\right) \\
& =P R_{C}(T) R_{C}\left(K_{1,1,0,2}\right)=P R_{C}\left(t_{2}\right)
\end{aligned}
$$

with

$$
t_{2}(\psi ; \eta)=\int T\left(\psi ; \eta^{\prime}\right) K_{1,1,0,2}\left(\psi ; \eta^{\prime}, \xi^{\prime}, \eta\right) d \mu_{C}\left(\eta^{\prime}\right)
$$

Definition VII.14. The two-legged tails $T_{\ell}(K)$ are recursively defined by

$$
\begin{aligned}
T_{1}(K)(\psi ; \eta) & =K_{2,0,0,2}\left(\psi ; \xi, \xi^{\prime}, \eta\right), \\
T_{\ell+1}(K) & =T_{\ell} \circ K \quad \text { for } \ell \geq 1 .
\end{aligned}
$$

Remark VII.15. (i) Clearly $T_{\ell}(K)$ has two external legs. Using Prop. II.33 one proves by induction that for $\alpha \geq 2$,

$$
N\left(T_{\ell}(K)\right) \leq \frac{1}{\alpha^{2 \ell-2}} N(K)^{\ell} .
$$

(ii) If $K\left(\psi ; \xi, \xi^{\prime}, \eta\right)=U\left(\psi ; \xi+\xi^{\prime}+\eta\right)-U\left(\psi ; \xi+\xi^{\prime}\right)$ for some even Grassmann function $U(\psi ; \xi)$ then, by Remark VII.10, $T_{\ell}(K)=T_{\ell}(U)$, where $T_{\ell}(U)$ was defined in Def. VI.8.ii.

(iii) $T_{\ell}(K)$ depends only on the part of $K$ that has degree at most two in the variables $\xi^{\prime}, \eta$. 
Proposition VII.16. Assume that $K$ has degree at most two in the variables $\xi^{\prime}, \eta$. For each $\ell \geq 1$ there exists a one-legged tail $t_{1}$ and a two-legged tail $t_{2}$, each with at least three external legs such that for any $f(\psi ; \xi) \in \bigwedge_{A}\left(V^{\prime} \oplus V\right)$ the following holds: Set

$$
\begin{aligned}
f_{\ell}^{\prime}(\psi)= & P\left[\mathcal{R}_{K, C}^{\ell}(: f:)-R_{C}\left(T_{\ell}(K)\right)(: f:)-\frac{1}{2} \sum_{\substack{\ell^{\prime}, \ell^{\prime \prime} \geq 1 \\
\max \left\{\ell^{\prime}, \ell^{\prime \prime}\right\}=\ell}} R_{C}\left(T_{\ell^{\prime}}(K), T_{\ell^{\prime \prime}}(K)\right)(: f:)\right. \\
& \left.-R_{C}\left(t_{1}+t_{2}\right)(: f:)\right] .
\end{aligned}
$$

Then, if $\alpha \geq 2$ and $N(K ; 2 \alpha)_{0}<\alpha^{2}$,

$$
N_{\text {impr }}\left(f_{\ell}^{\prime} ; \alpha\right) \leq J \frac{2^{7}}{\alpha^{2 \ell+6}} N(f ; 2 \alpha) \frac{N(K ; 2 \alpha)^{\ell+1}}{\left[1-\frac{1}{\alpha^{2}} N(K ; 2 \alpha)\right]^{\ell-1}} .
$$

Proof by induction on $\ell$. Set $N_{K}=N(K ; 2 \alpha)$. Since $K$ has degree at most two in the variables $\xi^{\prime}, \eta$ and degree at least four overall, for $\ell=1$,

$$
\begin{aligned}
P \mathcal{R}_{K, C}= & P R_{C}\left(K_{\cdot, 0,0,2}+K_{\cdot, 0,0,1}\right)+\frac{1}{2} P R_{C}\left(K_{2,0,1,1}, K_{2,0,1,1}\right) \\
& +\frac{1}{2} P R_{C}\left(K_{2,0,0,2}, K_{2,0,0,2}\right) \\
= & P R_{C}\left(T_{1}(K)\right)+P R_{C}\left(t_{1}+t_{2}\right)+\frac{1}{2} P R_{C}\left(T_{1}(K), T_{1}(K)\right)
\end{aligned}
$$

with

$$
\begin{aligned}
t_{1}= & K_{3,0,0,1}+K_{4,0,0,1}, \\
t_{2}= & K_{3,0,0,2}+K_{4,0,0,2}+\frac{1}{2} \int K_{2,0,1,1}\left(\psi ; \xi, \xi^{\prime}, \eta\right) \\
& \times K_{2,0,1,1}\left(\psi ; \xi, \xi^{\prime}, \eta\right) d \mu_{C}\left(\xi^{\prime}\right) .
\end{aligned}
$$

Now assume that the statement of the lemma is true for $\ell$. By the induction hypothesis there exist a one-legged tail $t_{1}$ and a two-legged tail $t_{2}$, each with at least three external legs, such that

$$
P\left[\mathcal{R}_{K, C}^{\ell+1}(: f:)\right]=P\left[\mathcal{R}_{K, C}^{\ell}\left(\mathcal{R}_{K, C}(: f:)\right)\right]
$$

differs from

$$
\begin{aligned}
& P\left[R_{C}\left(T_{\ell}(K)\right)\left(\mathcal{R}_{K, C}(: f:)\right)+\frac{1}{2} \sum_{\substack{\ell^{\prime}, \ell^{\prime \prime} \geq 1 \\
\max \left\{\ell^{\prime}, \ell^{\prime \prime}\right\}=\ell}} R_{C}\left(T_{\ell^{\prime}}(K), T_{\ell^{\prime \prime}}(K)\right)\left(\mathcal{R}_{K, C}(: f:)\right)\right. \\
& \left.\quad+R_{C}\left(t_{1}+t_{2}\right)\left(\mathcal{R}_{K, C}(: f:)\right)\right]
\end{aligned}
$$

by a function $g_{0}(\psi)$ with

$$
\begin{aligned}
N_{\text {impr }}\left(g_{0}\right) & \leq \frac{2^{7}}{\alpha^{2 \ell+6}} J \frac{N_{K}^{\ell+1}}{\left[1-\frac{1}{\alpha^{2}} N_{K}\right]^{\ell-1}} N(\tilde{f} ; 2 \alpha) \\
& \leq \frac{2^{7}}{\alpha^{2 \ell+6}} J \frac{N_{K}^{\ell+1}}{\left[1-\frac{1}{\alpha^{2}} N_{K}\right]^{\ell-1}} \frac{1}{2 \alpha^{2}} \frac{N_{K}}{1-\frac{1}{2 \alpha^{2}} N_{K}} N(f ; 2 \alpha) \\
& \leq \frac{2^{6}}{\alpha^{2 \ell+8}} J \frac{N_{K}^{\ell+2}}{\left[1-\frac{1}{\alpha^{2}} N_{K}\right]^{\ell}} N(f ; 2 \alpha),
\end{aligned}
$$


where $: \tilde{f}:=\mathcal{R}_{K, C}(: f:)$. Here, we used Lemma III.8, with $\alpha$ replaced by $2 \alpha$, to estimate $N(\tilde{f} ; 2 \alpha)$.

By Lemma VII.11, there exists a one-legged tail $t_{11}$ and a two-legged tail $t_{21}$, with at least three external legs each, such that

$$
\begin{aligned}
P\left[R_{C}\left(T_{\ell}(K)\right)\left(\mathcal{R}_{K, C}(: f:)\right)\right]= & P\left[R_{C}\left(T_{\ell+1}(K)\right)(: f:)\right] \\
& +P\left[R_{C}\left(T_{\ell+1}(K), T_{1}(K)\right)(: f:)\right] \\
& +P\left[R_{C}\left(t_{11}+t_{21}\right)(: f:)+g_{1}(\psi)\right] \\
= & P\left[R_{C}\left(T_{\ell+1}(K)\right)(: f:)\right] \\
& +\frac{1}{2} P\left[R_{C}\left(T_{\ell+1}(K), T_{1}(K)\right)(: f:)\right] \\
& +\frac{1}{2} P\left[R_{C}\left(T_{1}(K), T_{\ell+1}(K)\right)(: f:)\right] \\
& +P\left[R_{C}\left(t_{11}+t_{21}\right)(: f:)+g_{1}(\psi)\right]
\end{aligned}
$$

with

$$
\begin{aligned}
N_{\text {impr }}\left(g_{1}\right) & \leq \frac{2^{6} J}{\alpha^{10}} N(f ; 2 \alpha) N_{K}^{2} N\left(T_{\ell}(K) ; 2 \alpha\right) \\
& \leq \frac{2^{6} J}{\alpha^{2 \ell+8}} N(f ; 2 \alpha) N_{K}^{\ell+2} .
\end{aligned}
$$

Here, we used Remark VII.15 to bound $N\left(T_{\ell}(K) ; 2 \alpha\right)$ by $\frac{1}{\alpha^{2 \ell-2}} N_{K}^{\ell}$.

Similarly, for $\ell^{\prime}, \ell^{\prime \prime} \geq 1$ with $\max \left\{\ell^{\prime}, \ell^{\prime \prime}\right\}=\ell$, by Lemma VII.12, there exists a one-legged tail $t_{12}^{\left(\ell^{\prime}, \ell^{\prime \prime}\right)}$ and a two-legged tail $t_{22}^{\left(\ell^{\prime}, \ell^{\prime \prime}\right)}$ with at least three external legs such that

$$
\begin{aligned}
P\left[R_{C}\left(T_{\ell^{\prime}}(K), T_{\ell^{\prime \prime}}(K)\right)\left(\mathcal{R}_{K, C}(: f:)\right)\right]= & P\left[R_{C}\left(T_{\ell^{\prime}+1}(K), T_{\ell^{\prime \prime}+1}(K)\right)(: f:)\right] \\
& +P\left[R_{C}\left(t_{12}^{\left(\ell^{\prime}, \ell^{\prime \prime}\right)}+t_{22}^{\left(\ell^{\prime}, \ell^{\prime \prime}\right)}\right)(: f:)+g_{2, \ell^{\prime}, \ell^{\prime \prime}}(\psi)\right.
\end{aligned}
$$

with

$$
\begin{aligned}
N_{\text {impr }}\left(g_{2, \ell^{\prime}, \ell^{\prime \prime}}\right) & \leq \frac{2^{6} J}{\alpha^{12}} N(f ; 2 \alpha) N_{K}^{2} N\left(T_{\ell^{\prime}}(K) ; 2 \alpha\right) N\left(T_{\ell^{\prime \prime}}(K) ; 2 \alpha\right) \\
& \leq J \frac{2^{6}}{\alpha^{2\left(\ell^{\prime}+\ell^{\prime \prime}\right)+8}} N(f ; 2 \alpha) N_{K}^{\ell^{\prime}+\ell^{\prime \prime}+2} .
\end{aligned}
$$

By Lemma VII.13, there exists a two-legged tail $t_{23}$ with at least four external legs such that

$$
P\left[R_{C}\left(t_{1}\right)\left(\mathcal{R}_{K, C}(: f:)\right)\right]=P\left[R_{C}\left(t_{23}\right)(: f:)\right] .
$$

Finally, by Lemma VII.11

$$
P\left[R_{C}\left(t_{2}\right)\left(\mathcal{R}_{K, C}(: f:)\right)\right]=P\left[R_{C}\left(t_{2} \circ K+t_{14}+t_{24}\right)(: f:)\right],
$$

where $t_{14}, t_{24}$ are one- resp. two-legged tails with at least three external legs.

Combining the results above, we see that

$$
\begin{aligned}
P\left[\mathcal{R}_{K, C}^{\ell+1}(: f:)\right]= & f_{\ell+1}^{\prime}(\psi)+P\left[R_{C}\left(T_{\ell+1}(K)\right)(: f:)\right. \\
& \left.+\frac{1}{2} \sum_{\substack{\ell^{\prime} \ell^{\prime \prime \prime} \\
\max \left\{\ell^{\prime}, \ell^{\prime \prime}\right)=\ell+1}} R_{C}\left(T_{\ell^{\prime}}(K), T_{\ell^{\prime \prime}}(K)\right)(: f:)+R_{C}\left(t_{1}^{\prime}+t_{2}^{\prime}\right)(: f:)\right]
\end{aligned}
$$


with one- resp. two-legged tails $t_{1}^{\prime}, t_{2}^{\prime}$ with at least three external legs, and

$$
f_{\ell+1}^{\prime}=g_{0}+g_{1}+\frac{1}{2} \sum_{\substack{\left.\ell^{\prime}, \ell^{\prime \prime} \geq 1 \\ \max \ell^{\prime}, \ell^{\prime \prime}\right\}=\ell}} g_{2, \ell^{\prime}, \ell^{\prime \prime}}
$$

By the triangle inequality

$$
\begin{aligned}
N_{\text {impr }}\left(f_{\ell+1}^{\prime}\right) & \leq N_{\text {impr }}\left(g_{0}\right)+N_{\text {impr }}\left(g_{1}\right)+\frac{1}{2} \sum_{\substack{\left.\ell^{\prime}, \ell^{\prime \prime} \geq 1 \\
\max \ell^{\prime}, \ell^{\prime \prime}\right]=\ell}} N_{\text {impr }}\left(g_{2, \ell^{\prime}, \ell^{\prime \prime}}\right) \\
& \leq \frac{2^{6}}{\alpha^{2 \ell+8}} J N(f ; 2 \alpha) N_{K}^{\ell+2}\left[\frac{1}{\left[1-\frac{1}{\alpha^{2}} N_{K}\right]^{\ell}}+1+\sum_{\ell^{\prime}=1}^{\ell} \frac{N_{K}^{\ell^{\prime}}}{\alpha^{2 \ell^{\prime}}}\right] \\
& \leq \frac{2^{6}}{\alpha^{2 \ell+8}} J N(f ; 2 \alpha) N_{K}^{\ell+2}\left[\frac{1}{\left[1-\frac{1}{\alpha^{2}} N_{K}\right]^{\ell}}+\sum_{\ell^{\prime}=0}^{\infty}\left(\frac{N_{K}}{\alpha^{2}}\right)^{\ell^{\prime}}\right] \\
& \leq \frac{2^{7}}{\alpha^{2 \ell+8}} J N(f ; 2 \alpha) \frac{N_{K}^{\ell+2}}{\left[1-\frac{1}{\alpha^{2}} N_{K}\right]^{\ell} .}
\end{aligned}
$$

Corollary VII.17. Let $K\left(\psi ; \xi, \xi^{\prime}, \eta\right)$ be an even Grassmann function that has degree at most two in the variables $\xi^{\prime}, \eta$, degree at least one in $\eta$ and degree at least four in the variables $\psi, \xi, \xi^{\prime}, \eta$. Furthermore let $f(\psi ; \xi)$ be a Grassmann function of degree at least four in the variables $\psi, \xi$. Set

$$
\begin{aligned}
h(\psi)= & P\left[\frac{1}{1-\mathcal{R}_{K, C}}(: f:)-\sum_{\ell=0}^{\infty} R_{C}\left(T_{\ell}(K)\right)(: f:)\right. \\
& \left.-\frac{1}{2} \sum_{\ell^{\prime}, \ell^{\prime \prime} \geq 1} R_{C}\left(T_{\ell^{\prime}}(K), T_{\ell^{\prime \prime}}(K)\right)(: f:)\right] .
\end{aligned}
$$

If $\alpha \geq 2$ and $N(K ; 2 \alpha)_{0}<\frac{\alpha^{2}}{2}$, then

$$
N_{\text {impr }}(h) \leq \frac{2^{7} J}{\alpha^{8}} N(f ; 2 \alpha) \frac{N(K ; 2 \alpha)^{2}}{1-\frac{2}{\alpha^{2}} N(K ; 2 \alpha)} .
$$

Proof. By Prop. VII.16,

$$
\begin{aligned}
N_{\text {impr }}(h) & \leq \sum_{\ell=1}^{\infty} N_{\text {impr }}\left(f_{\ell}^{\prime}\right) \\
& \leq \frac{2^{7} J}{\alpha^{8}} N(f ; 2 \alpha) N(K ; 2 \alpha)^{2} \sum_{\ell=1}^{\infty}\left[\frac{1}{\alpha^{2}} \frac{N(K ; 2 \alpha)}{1-\frac{1}{\alpha^{2}} N(K ; 2 \alpha)}\right]^{\ell-1} \\
& =\frac{2^{7} J}{\alpha^{8}} N(f ; 2 \alpha) N(K ; 2 \alpha)^{2}\left[1-\frac{1}{\alpha^{2}} \frac{N(K ; 2 \alpha)}{1-\frac{1}{\alpha^{2}} N(K ; 2 \alpha)}\right]^{-1} \\
& =\frac{2^{7} J}{\alpha^{8}} N(f ; 2 \alpha) N(K ; 2 \alpha)^{2} \frac{1-\frac{1}{\alpha^{2}} N(K ; 2 \alpha)}{1-\frac{2}{\alpha^{2}} N(K ; 2 \alpha)} \\
& \leq \frac{2^{7} J}{\alpha^{8}} N(f ; 2 \alpha) \frac{N(K ; 2 \alpha)^{2}}{1-\frac{2}{\alpha^{2}} N(K ; 2 \alpha)} .
\end{aligned}
$$


Proof of Theorem VI.10 in the case $D=0$. Set $K\left(\psi ; \xi, \xi^{\prime}, \eta\right)=\hat{U}\left(\psi ; \xi+\xi^{\prime}+\eta\right)-$ $\hat{U}\left(\psi ; \xi+\xi^{\prime}\right)$. By Theorem III.2 and Prop. III.5,

$$
P f^{\prime}=P \mathcal{S}_{U, C}(: \hat{f}:)=P\left[\frac{1}{\mathbb{1}-\mathcal{R}_{K, C}}(: \hat{f}:)\right] .
$$

Observe that $K$ has degree at least one in $\eta$ and four overall and that, by part i of Remark II.24 (twice),

$$
N(K ; 2 \alpha)_{\mathbf{0}} \leq N\left(\hat{U}\left(\psi ; \xi+\xi^{\prime}+\eta\right) ; 2 \alpha\right)_{\mathbf{0}} \leq N(\hat{U}(\psi ; \xi) ; 8 \alpha)_{\mathbf{0}}<\frac{\alpha}{8} .
$$

Decompose

$$
K\left(\psi ; \xi, \xi^{\prime}, \eta\right)=K^{\prime}\left(\psi ; \xi, \xi^{\prime}, \eta\right)+K^{\prime \prime}\left(\psi ; \xi, \xi^{\prime}, \eta\right),
$$

where $K^{\prime}$ has degree at most two in the variables $\xi^{\prime}, \eta$ and $K^{\prime \prime}$ has degree at least three in the variables $\xi^{\prime}, \eta$. By Cor. VII.5 and Cor. VII.17 there exists a Grassmann function $g(\psi)$ with

$$
\begin{aligned}
N_{\text {impr }}(g) & \leq \frac{2^{5} J}{\alpha^{6}} N(\hat{f} ; 2 \alpha) N(K ; 2 \alpha)\left[\frac{1}{1-\frac{6}{\alpha} N(K ; 2 \alpha)}+\frac{\frac{4}{\alpha^{2}} N(K ; 2 \alpha)}{1-\frac{2}{\alpha^{2}} N(K ; 2 \alpha)}\right] \\
& \leq \frac{2^{5} J}{\alpha^{6}} N(\hat{f} ; 2 \alpha) N(K ; 2 \alpha) \frac{1+\frac{2}{\alpha} N(K ; 2 \alpha)}{1-\frac{6}{\alpha} N(K ; 2 \alpha)} \\
& \leq \frac{2^{5} J}{\alpha^{6}} N(\hat{f} ; 2 \alpha) N(K ; 2 \alpha) \frac{1}{1-\frac{6}{\alpha} N(K ; 2 \alpha)} \frac{1}{1-\frac{2}{\alpha} N(K ; 2 \alpha)} \\
& \leq \frac{2^{5} J}{\alpha^{6}} N(\hat{f} ; 2 \alpha) N(K ; 2 \alpha) \frac{1}{1-\frac{8}{\alpha} N(K ; 2 \alpha)} \\
& \leq \frac{2^{5} J}{\alpha^{6}} N(\hat{f} ; 2 \alpha) \frac{N(\hat{U} ; 8 \alpha)}{1-\frac{8}{\alpha} N(\hat{U} ; 8 \alpha)}
\end{aligned}
$$

and

$$
P f^{\prime}=P\left[\hat{f}(\psi, 0)+\sum_{\ell=1}^{\infty} R_{C}\left(T_{\ell}(K)\right)(: \hat{f}:)+\frac{1}{2} \sum_{\ell, \ell^{\prime}} R_{C}\left(T_{\ell}(K), T_{\ell^{\prime}}(K)\right)(: \hat{f}:)\right]+g .
$$

We have used that $P: \hat{f}(\psi, \xi):=P \hat{f}(\psi, 0)$ and $T_{\ell}\left(K^{\prime}\right)=T_{\ell}(K)$. Since $\hat{f}$ and $K$ have degree at least four overall,

$$
P R_{C}\left(T_{\ell}(K)\right)(: \hat{f}:)=T_{\ell}(K) \circ T_{1}(\hat{f})
$$

(where the $\circ$ composition was defined in Def. VI.5.ii) and by Remark VI.9,

$$
P\left[R_{C}\left(T_{\ell}(K), T_{\ell^{\prime}}(K)\right)(: \hat{f}:)\right]=T_{\ell}(K) \circ \operatorname{Rung}(\hat{f}) \circ T_{\ell^{\prime}}(K) .
$$

Furthermore, by part (ii) of Remark VII.15, $T_{\ell}(K)=T_{\ell}(\hat{U})$. Thus

$$
P f^{\prime}=P \hat{f}(\psi, 0)+\sum_{\ell=1}^{\infty} T_{\ell}(\hat{U}) \circ T_{1}(\hat{f})+\frac{1}{2} \sum_{\ell, \ell^{\prime} \geq 1} T_{\ell}(\hat{U}) \circ \operatorname{Rung}(\hat{f}) \circ T_{\ell^{\prime}}(\hat{U})+g .
$$




\section{The Enlarged Algebra}

The estimate of Theorem IV.1 on $W^{\prime}(\psi)$, defined by

$$
: W^{\prime}(\psi):_{\psi, D}=\Omega_{C}\left(: W:_{\psi, C+D}\right)
$$

was proven in the following way. We applied the results of Theorem II.28, combined with the estimates on Wick ordering (Cor. II.32) to get estimates on

$$
W^{\prime \prime}(\psi)=\Omega_{C}(: W: \psi, C+D)=\Omega_{C}\left(: W: \psi, D:{ }_{\psi, C}\right)
$$

in terms of the norm of $W$. Then we used Cor. II.32 again to estimate the norm of $W^{\prime}$ in terms of the norm of $W^{\prime \prime}$. The transition from $W^{\prime \prime}$ to $W^{\prime}=: W^{\prime \prime}:{ }_{\psi,-D}$ creates new two and four legged vertices, whose improved norm cannot be estimated by the technique of Sect. VI. This transition also creates new ladder diagrams.

The same difficulty would occur if we tried to reduce the general case of Theorem VI.10 to the special case $D=0$ by Wick ordering $\mathcal{S}_{U, C}(f)$ at the end of the construction. Therefore we monitor the Wick ordering with respect to $D$ throughout the construction. To do this we introduce fields $\zeta, \zeta^{\prime}, \varphi$ for these Wick contractions and use an analogue of the operator $\mathcal{R}_{K, C}$ of Def. III.4.

Definition VIII.1. (i) Let $\bar{K}_{1}\left(\psi ; \zeta, \zeta^{\prime}, \varphi ; \xi, \xi^{\prime}, \eta\right), \cdots, \bar{K}_{\ell}\left(\psi ; \zeta, \zeta^{\prime}, \varphi ; \xi, \xi^{\prime}, \eta\right)$ be even Grassmann functions with $\bar{K}_{i}\left(\psi ; \zeta, \zeta^{\prime}, \varphi ; \xi, \xi^{\prime}, 0\right)=0$. For a Grassmann function $g(\psi ; \zeta ; \xi)$ we define

$$
\begin{aligned}
Q\left(\bar{K}_{1}, \cdots, \bar{K}_{\ell}\right)(g)(\psi ; \zeta ; \xi)= & : \iint:\left(\prod_{i=1}^{\ell}: \bar{K}_{i}\left(\psi ; \zeta, \zeta^{\prime}, \varphi ; \xi, \xi^{\prime}, \eta\right):_{\substack{\zeta^{\prime}, D \\
\xi^{\prime}, C}}\right):_{\varphi, D} \\
& : g(\psi ; \zeta+\varphi ; \eta): \varphi, D d \mu_{D}\left(\zeta^{\prime}, \varphi\right) d \mu_{C}\left(\xi^{\prime}, \eta\right):_{\xi, C} .
\end{aligned}
$$

The structure of $Q$ is illustrated in the figure

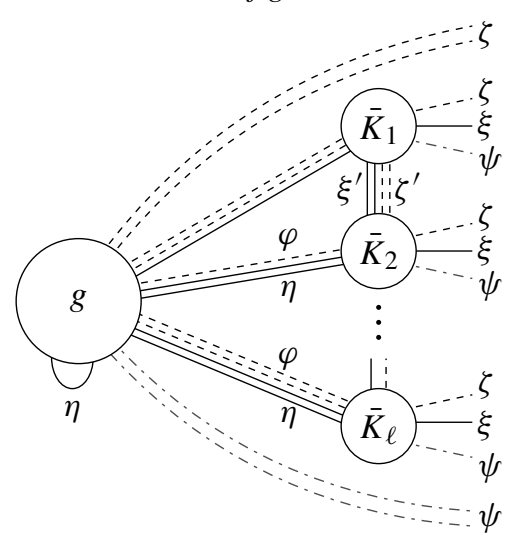

(ii) For a Grassmann function $\bar{K}\left(\psi ; \zeta, \zeta^{\prime}, \varphi ; \xi, \xi^{\prime}, \eta\right)$ with $\bar{K}\left(\psi ; \zeta, \zeta^{\prime}, \varphi ; \xi, \xi^{\prime}, 0\right)=0$ we define the operator $\mathcal{Q}_{\bar{K}}$ by

$$
\mathcal{Q}_{\bar{K}}(g)=\sum_{\ell=1}^{\infty} \frac{1}{\ell !} Q(\bar{K}, \cdots, \bar{K})(g) .
$$


In this definition, the fields $\xi, \xi^{\prime}, \eta$ involving the covariance $C$ are treated in the same way as in Sect. III. The fields $\varphi$ are analogous to the fields $\eta$ and describe Wick contractions between $g$ and the $K_{i}$. The fields $\zeta^{\prime}$ are analogous to the fields $\xi^{\prime}$ and describe Wick contractions among $K_{i}$. Similar to the $\xi$ fields, the fields $\zeta$ are not integrated out and are later used for Wick contractions in further applications of $\mathcal{Q}_{\bar{K}}$. In contrast to the $\xi$-fields, however, also Wick contractions between fields of $g$ and kernels appearing in future applications of $\mathcal{Q}_{\bar{K}}$ have to be allowed. This is the reason for the field $\zeta$ that appears in the term $g(\psi ; \zeta+\varphi ; \eta):_{\varphi, D}$ in Def. VIII.1.i.

Definition VIII.1.i of $Q$ was chosen so that

$$
: Q\left(\bar{K}_{1}, \cdots, \bar{K}_{\ell}\right)(g)_{\zeta, D}=R\left(: K_{1}:_{\zeta, D}, \cdots,: K_{\ell}:_{\zeta, D}\right)\left(: g:_{\zeta, D}\right)
$$

when $\bar{K}_{i}\left(\psi ; \zeta, \zeta^{\prime}, \varphi ; \xi, \xi^{\prime}, \eta\right)=K_{i}\left(\psi ; \zeta+\zeta^{\prime}+\varphi ; \xi, \xi^{\prime}, \eta\right)$. This formula is proven in Lemma VIII.12.i. ${ }^{1}$ Consequently

$$
: \mathcal{Q}_{\bar{K}}^{n}(h)_{\zeta, D}=\mathcal{R}_{: K:_{\zeta, D}}^{n}\left(: h:_{\zeta, D}\right)
$$

for $n=0,1,2, \cdots$. From this one can deduce, as in Prop. VIII.9, that

$$
\mathcal{S}_{U, C}(: f: \psi, D)=: \int \sum_{n=0}^{\infty} \mathcal{Q}_{\bar{K}}^{n}(h)(\psi ; 0 ; \xi) d \mu_{C}(\xi){ }_{\cdot \psi, D},
$$

where, given an even Grassmann function $\hat{U}(\psi ; \xi)$ and a Grassmann function $f(\psi ; \xi)$,

$$
\begin{aligned}
U(\psi ; \xi)= & : \hat{U}(\psi ; \xi): \underset{\substack{\xi, C \\
\psi, D}}{ } \\
h(\psi ; \zeta ; \xi)= & f(\psi+\zeta ; \xi), \\
\bar{K}\left(\psi ; \zeta, \zeta^{\prime}, \varphi ; \xi, \xi^{\prime}, \eta\right)= & \hat{U}\left(\psi+\zeta+\zeta^{\prime}+\varphi ; \xi+\xi^{\prime}+\eta\right) \\
& -\hat{U}\left(\psi+\zeta+\zeta^{\prime}+\varphi ; \xi+\xi^{\prime}\right) .
\end{aligned}
$$

However, this formula would not be good enough to get an estimate on $\mathcal{S}_{U, C}\left(: f:_{C+D}\right)$. At each application of $\mathcal{Q}_{\bar{K}}$, the passage from $g(\psi ; \zeta ; \xi)$ to $g(\psi ; \zeta+\varphi ; \eta)$, that is the separation between the $D$ - fields that are to be Wick contracted at the present step and those that are to be Wick contracted at a future step, leads to a deterioration in the norm:

$$
N(g(\psi ; \zeta+\varphi ; \eta) ; \alpha) \leq N(g(\psi ; \zeta ; \xi) ; 2 \alpha)
$$

by Remark II.24. In iterated applications of $\mathcal{Q}_{\bar{K}}$ this deterioration in the norm would build up excessively. For this reason, we avoid Wick contractions at most steps of the construction and perform them only before and during steps in which overlapping loops are exploited. For bookkeeping of the partially Wick ordered fields in intermediate steps we introduce an enlarged algebra.

\footnotetext{
1 To see this, choose $f=: g:_{\zeta, D}$.
} 
VIII.1. Definition of the Enlarged Algebra.

Definition VIII.2. (i) $A \mathbb{Z}_{2}-$ graded vector space is a complex vector space $E$, together with a decomposition $E=E_{+} \oplus E_{-}$. The elements of $E_{+}$are called even, the elements of $E_{-}$odd. A graded vector space is a complex vector space $E$, together with a decomposition $E=\bigoplus_{m=0}^{\infty} E_{m}$. Every graded vector space is considered as a $\mathbb{Z}_{2}$-graded vector space with

$$
E_{+}=\bigoplus_{r \text { even }} E_{m} \quad E_{-}=\bigoplus_{r \text { odd }} E_{m} .
$$

(ii) If $E$ is a $\left(\mathbb{Z}_{2}-\right)$ graded vector space, the tensor algebra $T(E)$ has a natural $\left(\mathbb{Z}_{2}-\right)$ grading. The symmetric superalgebra over $E$ is denoted $S(E)$ and is defined as the quotient of $T(E)$ by the two sided ideal $I(E)$ generated by

$$
\begin{array}{ll}
a \otimes b-b \otimes a & \text { with } a \in E_{+} \text {or } b \in E_{+}, \\
a \otimes b+b \otimes a & \text { with } a, b \in E_{-} .
\end{array}
$$

It is a superalgebra, and it is a graded superalgebra if $E$ is a graded vector space (see $[B S])$.

Example VIII.3. Let $E$ be a complex vector space. Setting $E_{+}=\{0\}, E_{-}=E$, we give $E$ the structure of a $\mathbb{Z}_{2}$-graded vector space in which all elements are odd. Then the symmetric superalgebra over $E$ is the Grassmann algebra $\bigwedge E$ over $E$. Setting $E_{+}=E, E_{-}=\{0\}, S(E)$ is the classical symmetric algebra $\mathcal{S}(E)$ over $E$.

Remark VIII.4. There is a natural isomorphism $\iota$ between $S(E)$ and the algebra $\mathcal{S}\left(E_{+}\right) \otimes$ $\bigwedge E_{-}$, constructed in the following way:

Observe that

$$
T(E) \cong \bigoplus_{\substack{m_{1}, n_{1}, \cdots, m_{r}, n_{r} \geq 0 \\ n_{1}, m_{2}, \cdots, n_{r}, m_{r} \geq 1}} E_{+}^{\otimes m_{1}} \otimes E_{-}^{\otimes n_{1}} \otimes \cdots \otimes E_{+}^{\otimes m_{r}} \otimes E_{-}^{\otimes n_{r}} .
$$

Define the algebra homomorphism $\iota^{\prime}: T(E) \rightarrow \mathcal{S}\left(E_{+}\right) \otimes \wedge E_{-}$by

$$
\iota^{\prime}\left(v_{1}^{+} \otimes v_{1}^{-} \otimes \cdots \otimes v_{r}^{+} \otimes v_{r}^{-}\right)=\left(v_{1}^{+} \cdot \ldots \cdot v_{r}^{+}\right) \otimes\left(v_{1}^{-} \cdot \ldots \cdot v_{r}^{-}\right)
$$

for $v_{i}^{+} \in E_{+}^{\otimes m_{i}}, v_{i}^{-} \in E_{-}^{\otimes m_{i}}$. Clearly $I(E)$ lies in the kernel of $\iota^{\prime}$, so $\iota^{\prime}$ induces an algebra homomorphism $\iota: S(E) \rightarrow \mathcal{S}\left(E_{+}\right) \otimes \wedge E_{-}$. One can check that $\iota$ is an isomorphism.

As $\mathcal{S}(E)$ and $\bigwedge E$ are subalgebras of the tensor algebra $T(E), S(E)$ can also be viewed as a subalgebra of $T(E) \otimes T(E) \cong T(E)$.

Definition VIII.5. (i) Let $E$ be a complex vector space. The symmetric superalgebra $S(\bigwedge E)$ over $\bigwedge E$ (considered as a graded vector space) is called the enlarged algebra / $\mathrm{E}$ over $E$. It is a graded superalgebra. 
(ii) Multiplication

$$
f_{1} \otimes \cdots \otimes f_{r} \longmapsto f_{1} \cdots f_{r}
$$

defines an algebra homomorphism from the tensor algebra $T(\bigwedge E)$ to $\bigwedge E$. The ideal I $(\bigwedge E)$ of part (ii) of Def. VIII.2 lies in the kernel of this homomorphism. Therefore it induces an algebra homomorphism

$$
\mathrm{Ev}: / \bigwedge E \rightarrow \bigwedge E
$$

called the evaluation map. It is graded, that is

$$
\operatorname{Ev}(x) \in \bigwedge^{m} E \text { when } x \in(/ \bigwedge E)_{m} \text {. }
$$

(ii) If $A$ is a superalgebra, we define the enlarged algebra over $E$ with coefficients in $A$ as the tensor product

$$
/ \bigwedge_{A} E=A \otimes / \bigwedge E
$$

in the sense of Def. II.1.iv. The evaluation map extends by A-linearity to an algebra homomorphism

$$
\mathrm{Ev}: / \bigwedge_{A} E \longrightarrow \bigwedge_{A} E
$$

Remark VIII.6. (i) $\bigwedge E$ is identified with the subspace $(/ \bigwedge V)_{1}$ of $/ \bigwedge V$.

(ii) $\operatorname{Ev}(x \cdot y)=\operatorname{Ev}(x \cdot \operatorname{Ev}(y))=\operatorname{Ev}(\operatorname{Ev}(x) \cdot y)$ for all $x, y \in / \bigwedge V$.

(iii) By Remark VIII.4, there is a natural inclusion of $/ \bigwedge E=S(\bigwedge E)$ as a graded subalgebra of $T(\bigwedge E) \subset T(T(E)) \cong T(E)$.

VIII.2. Norm Estimates for the Enlarged Algebra. As in Subsect. III.3, let $A^{\prime}=\bigwedge_{A} V^{\prime}$ be the Grassmann algebra in the variables $\psi_{i}$ with coefficients in $A$. Furthermore let $E$ be a copy of $V$ with generators $\zeta_{i}$ corresponding to the fields $\psi_{i}$. We will use the enlarged algebra

$$
\mathcal{A}=/ \bigwedge_{A^{\prime}} E
$$

over $E$ with coefficients in $A^{\prime}$. Elements of $\mathcal{A}$ will be written as Grassmann functions $f(\psi ; \vec{\zeta})$. They are linear combinations of monomials of the form

$$
\psi_{i_{1}} \cdots \psi_{i_{n}}\left(\zeta_{j_{1}^{(1)}} \cdots \zeta_{j_{p_{1}}^{(1)}}\right) \otimes\left(\zeta_{j_{1}^{(2)}} \cdots \zeta_{j_{p_{2}}^{(2)}}\right) \otimes \cdots \otimes\left(\zeta_{j_{1}^{(r)}} \cdots \zeta_{j_{p r}^{(r)}}\right)
$$

The evaluation map Ev $: \mathcal{A} \rightarrow \bigwedge_{A^{\prime}} E \cong \bigwedge_{A}(V \oplus E)$ maps such a monomial to $\psi_{i_{1}} \cdots \psi_{i_{n}} \zeta_{j_{1}^{(1)}} \cdots \zeta_{j_{p_{1}}^{(1)}} \zeta_{j_{1}^{(2)}} \cdots \zeta_{j_{p r}^{(r)}}$. As in Remark VIII.6, the Grassmann algebra $\bigwedge_{A^{\prime}} E$ of Grassmann functions $g(\psi ; \zeta)$ is viewed as a subspace of $\mathcal{A}$.

The evaluation map Ev $: \mathcal{A} \rightarrow \bigwedge_{A^{\prime}} E \cong \bigwedge_{A}(V \oplus E)$ extends to a map

$$
\begin{aligned}
\mathrm{Ev}: \bigwedge_{\mathcal{A}}\left(V^{(1)} \oplus \cdots \oplus V^{(r)}\right) & \longrightarrow \bigwedge_{A^{\prime}}\left(E \oplus V^{(1)} \oplus \cdots \oplus V^{(r)}\right) \\
& \cong \bigwedge_{A}\left(V \oplus E \oplus V^{(1)} \oplus \cdots \oplus V^{(r)}\right),
\end{aligned}
$$

where $V^{(j)}, j=1, \cdots, r$ are copies of $V$ with generators $\xi^{(j)}$. 
Definition VIII.7. By Remark VIII.6.iii, $\mathcal{A}$ can be viewed as a graded subalgebra of $A^{\prime} \otimes T(E) \cong A^{\prime} \otimes T(V)$. Therefore the family $\|\cdot\|^{\prime}$ of seminorms on the spaces $A_{m}^{\prime} \otimes V^{\otimes n}$, introduced in Subsect. III.3, induces a family of symmetric seminorms on the spaces $\mathcal{A}_{m^{\prime}} \otimes V^{\otimes n^{\prime}}$, which we again denote by $\|\cdot\|^{\prime}$. This definition extends the definition of $\|\cdot\|^{\prime}$ given in Subsect. III.3. Also, $\mathfrak{c}$ is a contraction bound and $\mathrm{b}$ an integral bound for the covariance $C$ with respect to these norms.

Lemma VIII.8. Let $f \in \bigwedge_{\mathcal{A}}\left(V_{1} \oplus \cdots \oplus V_{r}\right)$. Then

$$
N^{\prime}(\operatorname{Ev}(f) ; \alpha) \leq N^{\prime}(f ; \alpha) .
$$

Proof. $\operatorname{Ev}(f)$ is obtained from $f$ by antisymmetrization.

VIII.3. Schwinger Functionals over the Extended Algebra. Recall that for any even $U(\psi ; \xi) \in \bigwedge_{A^{\prime}} V$, the Schwinger functional with respect to $U$ and $C$ is the map from $\bigwedge_{A^{\prime}} V$ to $A^{\prime}$ given by

$$
\mathcal{S}_{U, C}(f)(\psi)=\frac{1}{Z} \int e^{U(\psi ; \xi)} f(\psi ; \xi) d \mu_{C}(\xi), \quad Z=\int e^{U(\psi ; \xi)} d \mu_{C}(\xi) .
$$

Also recall that for an even Grassmann function $K\left(\psi, \vec{\zeta} ; \xi, \xi^{\prime}, \eta\right)$ in the variables $\xi, \xi^{\prime}, \eta$ over the extended algebra $\mathcal{A}$ and any Grassmann function $f(\psi ; \vec{\zeta} ; \xi) \in \bigwedge_{\mathcal{A}} V$,

$$
\mathcal{R}_{K, C}(f)=: \iint: e^{: K\left(\psi, \vec{\zeta} ; \xi, \xi^{\prime}, \eta\right): \xi^{\prime}, C}-1_{{ }_{\eta, C}} f(\psi ; \vec{\zeta} ; \eta) d \mu_{C}\left(\xi^{\prime}\right) d \mu_{C}(\eta){ }_{\xi, C} .
$$

Proposition VIII.9. Let

$$
U(\psi ; \xi)=: \hat{U}(\psi ; \xi): \underset{\xi, D}{\underset{\xi}{, C}}
$$

be even and set

$$
K\left(\psi, \zeta ; \xi, \xi^{\prime}, \eta\right)=: \hat{U}\left(\psi+\zeta ; \xi+\xi^{\prime}+\eta\right):_{\zeta, D}-: \hat{U}\left(\psi+\zeta ; \xi+\xi^{\prime}\right):_{\zeta, D}
$$

Let $f(\psi ; \xi) \in \bigwedge_{A^{\prime}} V$ and set $\tilde{f}(\psi ; \zeta ; \xi)=: f(\psi+\zeta ; \xi)_{\zeta, D} \in \bigwedge_{\mathcal{A}} V$. Then

$$
\mathcal{S}_{U, C}\left(: f:{ }_{\psi}, D\right)(\psi)=: \iint \operatorname{Ev}\left(\frac{1}{\mathbb{1}-\mathcal{R}_{K, C}}(\tilde{f})\right) d \mu_{C}(\xi) d \mu_{D}(\zeta)_{\cdot \psi, D}^{\cdot} .
$$

Observe that $\frac{1}{1-\mathcal{R}_{K, C}}(\tilde{f}) \in \bigwedge_{\mathcal{A}} V$, so that $\int \frac{1}{1-\mathcal{R}_{K, C}}(\tilde{f}) d \mu_{C}(\xi) \in \mathcal{A}$.

For the proof of this proposition we use

Lemma VIII.10. Let $f(\psi ; \xi)$ be a Grassmann function and set

$$
\tilde{f}(\psi ; \zeta ; \xi)=: f(\psi+\zeta ; \xi): \zeta, D
$$

Furthermore, let $n \geq 1$. 
(i) Let $\ell_{1}, \cdots, \ell_{n} \geq 1$ and let

$$
\hat{K}_{j i}\left(\psi ; \xi, \xi^{\prime}, \eta\right), \quad j=1, \cdots n, \quad i=1, \cdots, \ell_{j}
$$

be even Grassmann functions with coefficients in A. Let

$$
K_{j i}\left(\psi ; \zeta ; \xi, \xi^{\prime}, \eta\right)=: \hat{K}_{j i}\left(\psi+\zeta ; \xi, \xi^{\prime}, \eta\right):_{\zeta, D}
$$

considered as Grassmann functions in the variables $\xi, \xi^{\prime}, \eta$ with coefficients in the enlarged algebra $\mathcal{A}$. Then

$$
\begin{aligned}
& \left(\prod_{j=1}^{n} R_{C}\left(: \hat{K}_{j 1}: \psi, D, \cdots,: \hat{K}_{j \ell_{j}}: \psi, D\right)\right)(: f: \psi, D) \\
& =: \int \operatorname{Ev}\left(\prod_{j=1}^{n} R_{C}\left(K_{j 1}, \cdots, K_{j \ell_{j}}\right)(\tilde{f})\right) d \mu_{D}(\zeta)_{\cdot \psi, D}^{\cdot} .
\end{aligned}
$$

On the left-hand side of the equation above, the operator $R_{C}$ (defined in (III.2)) is considered over the algebra $A^{\prime}$, while on the right-hand side it is considered over the extended algebra $\mathcal{A}$.

(ii) Let $\hat{K}\left(\psi ; \xi, \xi^{\prime}, \eta\right)$ be an even Grassmann function and

$$
K\left(\psi ; \zeta ; \xi, \xi^{\prime}, \eta\right)=: \hat{K}\left(\psi+\zeta ; \xi, \xi^{\prime}, \eta\right):_{\zeta, D}
$$

Then

$$
\mathcal{R}_{: \hat{K}: \psi, D}^{n}\left(: f:{ }_{\psi}, D\right)=: \int \operatorname{Ev} \mathcal{R}_{K, C}^{n}(\tilde{f}) d \mu_{D}(\zeta)_{\cdot \psi, D}^{\cdot} .
$$

Proof. (i) Let

$$
h(\psi ; \xi)=: g(\psi ; \xi): \psi, D=\left(\prod_{j=1}^{n} R_{C}\left(: \hat{K}_{j 1}: \psi, D, \cdots,: \hat{K}_{j \ell_{j}}: \psi, D\right)\right)(: f: \psi, D) .
$$

By part (ii) of Prop. A.2, in the Grassmann algebra over $A$ with variables $\psi, \zeta, \xi$, $\xi^{\prime}, \eta$,

$$
h(\psi+\zeta ; \xi)=: g(\psi+\zeta ; \xi)_{\zeta, D}=\left(\prod_{j=1}^{n} R_{C}\left(K_{j 1}, \cdots, K_{j \ell_{j}}\right)\right)\left(: \tilde{f}_{: \zeta, D}\right) .
$$

Hence, by the construction of $\mathcal{A}$ and $\mathrm{Ev}$,

$$
h(\psi+\zeta ; \xi)=: g(\psi+\zeta ; \xi):_{\zeta, D}=\operatorname{Ev}\left[\left(\prod_{j=1}^{n} R_{C}\left(K_{j 1}, \cdots, K_{j \ell_{j}}\right)\right)\left(: \tilde{f}_{\zeta, D}\right)\right] .
$$

Therefore

$$
\begin{aligned}
g(\psi ; \xi) & =\int: g(\psi+\zeta ; \xi):_{\zeta, D} d \mu_{D}(\zeta) \\
& =\int \operatorname{Ev}\left(\prod_{j=1}^{n} R_{C}\left(K_{j 1}, \cdots, K_{j \ell_{j}}\right)(\tilde{f})\right) d \mu_{D}(\zeta) .
\end{aligned}
$$

(ii) follows from part (i) and Remark III.6. 
Proof of Proposition VIII.9. Set

$$
: \hat{K}\left(\psi ; \xi, \xi^{\prime}, \eta\right): \psi, D=: \hat{U}\left(\psi ; \xi+\xi^{\prime}+\eta\right): \psi, D-: \hat{U}\left(\psi ; \xi+\xi^{\prime}\right):_{\psi, D} .
$$

By Prop. III.5, with $\hat{U}(\xi)$ replaced by $: \hat{U}(\psi ; \xi): \psi, D$ and $K\left(\xi, \xi^{\prime}, \eta\right)$ replaced by $: \hat{K}\left(\psi ; \xi, \xi^{\prime}, \eta\right): \psi, D$, and part ii of Lemma VIII.10,

$$
\begin{aligned}
R_{U, C}^{n}(: f: \psi, D & =\mathcal{R}_{: \hat{K}: \psi, D}^{n}\left(: C: f_{\psi, D}\right) \\
& =: \int \operatorname{Ev}\left(\mathcal{R}_{K, C}^{n}\right)(\tilde{f}) d \mu_{D}(\zeta)_{\cdot \psi, D}^{.}
\end{aligned}
$$

By Theorem III.2,

$$
\begin{aligned}
\mathcal{S}_{U, C}\left(: f:_{\psi, D}\right)(\psi) & =\int \sum_{n \geq 0} R_{U, C}^{n}\left(: f:{ }_{*}, D\right. \\
& =: \int \operatorname{Ev}\left(\int \sum_{n \geq 0} \mathcal{R}_{K, C}^{n}(\tilde{f}) d \mu_{C}(\xi)\right) d \mu_{D}(\zeta)_{\cdot \psi, D}^{\cdot} \\
& =: \int \operatorname{Ev}\left(\int \frac{1}{1-\mathcal{R}_{K, C}}(\tilde{f}) d \mu_{C}(\xi)\right) d \mu_{D}(\zeta)_{\cdot \psi, D}^{\cdot}
\end{aligned}
$$

We use the extended algebra to give

VIII.4. A Second Proof of Theorem IV.1.

Proposition VIII.11. Let $\hat{U}(\psi ; \xi), \hat{f}(\psi ; \xi) \in \bigwedge_{A^{\prime}} V$ with $\hat{U}$ even. Set

$$
\begin{aligned}
& U(\psi ; \xi)=: \hat{U}(\psi ; \xi): \underset{\xi, C}{\psi, D}, \\
& f(\psi ; \xi)=: \hat{f}(\psi ; \xi): \underset{\xi, C}{\psi},
\end{aligned}
$$

Assume that $\mathrm{c}$ is a contraction bound for the covariance $C$ and $\mathrm{b}$ is an integral bound for $C$ and for $D$ and that $N(\hat{U} ; 8 \alpha)_{\mathbf{0}}<\frac{\alpha^{2}}{4}$. Then $\mathcal{S}_{U, C}(f)$ exists. If

$$
\mathcal{S}_{U, C}(f)=: f^{\prime}(\psi):_{\psi, D},
$$

then

$$
N\left(f^{\prime}(\psi)-\hat{f}(\psi ; 0) ; \alpha\right) \leq \frac{2}{\alpha^{2}} N(\hat{f} ; 4 \alpha) \frac{N(\hat{U} ; 8 \alpha)}{1-\frac{4}{\alpha^{2}} N(\hat{U} ; 8 \alpha)}
$$

Proof. As above, set

$$
\begin{aligned}
K\left(\psi ; \zeta ; \xi, \xi^{\prime}, \eta\right) & \left.=: \hat{U}\left(\psi+\zeta ; \xi+\xi^{\prime}+\eta\right)\right)_{\zeta, D}-: \hat{U}\left(\psi+\zeta ; \xi+\xi^{\prime}\right):_{\zeta, D}, \\
\tilde{f}(\psi ; \zeta ; \xi) & =: \hat{f}(\psi+\zeta ; \xi):_{\zeta, D} .
\end{aligned}
$$

By Remark II.24 and Cor. II.32,

$$
\begin{aligned}
N(K) & \leq N(\hat{U} ; 8 \alpha), \\
N(\tilde{f}) & \leq N(\hat{f} ; 4 \alpha) .
\end{aligned}
$$


By Cor. III.9, applied with $A$ replaced by $\mathcal{A}$,

$$
\frac{1}{1-\mathcal{R}_{K, C}}(: \tilde{f}: \xi, C)-: \tilde{f}: \xi, C=: g(\psi, \vec{\zeta} ; \xi): \xi, C
$$

with

$$
N^{\prime}(g) \leq \frac{2}{\alpha^{2}} N^{\prime}(\tilde{f}) \frac{N^{\prime}(K)}{1-\frac{4}{\alpha^{2}} N^{\prime}(K)}=\frac{2}{\alpha^{2}} N(\tilde{f}) \frac{N(K)}{1-\frac{4}{\alpha^{2}} N(K)} \leq \frac{2}{\alpha^{2}} N(\hat{f} ; 4 \alpha) \frac{N(\hat{U} ; 8 \alpha)}{1-\frac{4}{\alpha^{2}} N(\hat{U} ; 8 \alpha)} .
$$

Consequently, by Lemma II.31,

$$
\begin{aligned}
N^{\prime}\left[\int\left(\frac{1}{1-\mathcal{R}_{K, C}}(: \tilde{f}: \xi, C)-: \tilde{f}: \xi, C\right) d \mu_{C}(\xi)\right] & =N^{\prime}\left(\int: g(\psi ; \vec{\zeta} ; \xi): \xi, C d \mu_{C}(\xi)\right) \\
& \leq N^{\prime}(g(\psi ; \vec{\zeta} ; \xi)) \\
& \leq \frac{2}{\alpha^{2}} N(\hat{f} ; 4 \alpha) \frac{N(\hat{U} ; 8 \alpha)}{1-\frac{4}{\alpha^{2}} N(\hat{U} ; 8 \alpha)} .
\end{aligned}
$$

Observe that

$$
\int: \tilde{f}: \xi, C d \mu_{C}(\xi)=\tilde{f}(\psi ; \zeta ; 0)=: \hat{f}(\psi+\zeta ; 0):_{\zeta, D}
$$

Hence, by Prop. VIII.9, with $f(\psi ; \xi)$ replaced by : $\hat{f}(\psi ; \xi): \xi, C$, Lemma II.31 and Lemma VIII.8,

$$
\begin{aligned}
N\left(f^{\prime}-\hat{f}(\psi, 0)\right)= & N^{\prime}\left(f^{\prime}-\hat{f}(\psi, 0)\right) \\
= & N^{\prime}\left[\int \operatorname { E v } \left(\int \frac{1}{1-\mathcal{R}_{K, C}}(: \tilde{f}: \xi, C) d \mu_{C}(\xi)\right.\right. \\
& \left.\left.-: \hat{f}(\psi+\zeta, 0):_{\zeta, D}\right) d \mu_{D}(\zeta)\right] \\
\leq & N^{\prime}\left[\operatorname{Ev}\left(\int \frac{1}{1-\mathcal{R}_{K, C}}(: \tilde{f}: \xi, C) d \mu_{C}(\xi)-: \hat{f}(\psi+\zeta, 0): \xi, D\right)\right] \\
\leq & N^{\prime}\left[\int\left(\frac{1}{1-\mathcal{R}_{K, C}}(: \tilde{f}: \xi, C)-: \tilde{f}: \xi, C\right) d \mu_{C}(\xi)\right] \\
\leq & \frac{2}{\alpha^{2}} N(\hat{f} ; 4 \alpha) \frac{N(\hat{U} ; 8 \alpha)}{1-\frac{4}{\alpha^{2}} N(\hat{U} ; 8 \alpha)} .
\end{aligned}
$$

Proof of Theorem IV.1, again. By Remark III.1.i, with $W$ replaced by : $W:_{C+D}$,

$$
\begin{aligned}
: W^{\prime}(\psi)-W(\psi): \psi, D & =\Omega_{C}\left(: W:_{C+D}\right)(\psi)-: W(\psi): \psi, D \\
& =\int_{0}^{1}\left(\mathcal{S}_{t U, C}(U)-: W(\psi): \psi, D\right) d t \quad \bmod A_{0},
\end{aligned}
$$

where

$$
U(\psi ; \xi)=: W(\psi+\xi): \underset{\psi, D}{\xi, C}
$$


Define $f_{t}^{\prime}$ and $\hat{f}$ by

$$
\begin{aligned}
\hat{f}(\psi ; \xi) & =W(\psi+\xi) \\
\left.: f_{t}^{\prime}(\psi)\right)_{\psi, D} & =\mathcal{S}_{t U, C}(U) .
\end{aligned}
$$

Then

$$
W^{\prime}(\psi)-W(\psi)=\int_{0}^{1}\left(f_{t}^{\prime}(\psi)-\hat{f}(\psi, 0)\right) d t \quad \bmod A_{0} .
$$

By Prop. VIII.11, with $\hat{U}_{t}(\psi ; \xi)=t W(\psi+\xi)$,

$$
\begin{aligned}
N\left(W^{\prime}-W ; \alpha\right) & \leq \max _{0 \leq t \leq 1} N\left(f_{t}^{\prime}(\psi)-\hat{f}(\psi, 0) ; \alpha\right) \\
& \leq \frac{2}{\alpha^{2}} \max _{0 \leq t \leq 1} N(\hat{f} ; 4 \alpha) \frac{N\left(\hat{U}_{t} ; 8 \alpha\right)}{1-\frac{4}{\alpha^{2}} N\left(\hat{U}_{t} ; 8 \alpha\right)} \\
& \leq \frac{2}{\alpha^{2}} \frac{N(W ; 16 \alpha)^{2}}{1-\frac{4}{\alpha^{2}} N(W ; 16 \alpha)}
\end{aligned}
$$

for all $W$ with $N(W ; 16 \alpha)_{0}<\frac{\alpha^{2}}{4}$. Replacing $\alpha$ by $2 \alpha$ gives

$$
N\left(W^{\prime}-W ; 2 \alpha\right) \leq \frac{1}{2 \alpha^{2}} \frac{N(W ; 32 \alpha)^{2}}{1-\frac{1}{\alpha^{2}} N(W ; 32 \alpha)}
$$

for all $W$ with $N(W ; 32 \alpha)_{0}<\alpha^{2}$.

VIII.5. The Operator $Q$.

Lemma VIII.12. Let $f(\psi, \vec{\zeta} ; \xi)$ be a Grassmann function over the extended algebra $\mathcal{A}$ and set $: f^{\prime}(\psi, \zeta ; \xi): \zeta, D=\operatorname{Ev}(f)$.

(i) Let $K_{i}\left(\psi, \zeta ; \xi, \xi^{\prime}, \eta\right), i=1, \cdots, \ell$ be Grassmannfunctions with $K_{i}\left(\psi, \zeta ; \xi, \xi^{\prime}, 0\right)$ $=0$. Set

$$
\bar{K}_{i}\left(\psi ; \zeta, \zeta^{\prime}, \varphi ; \xi, \xi^{\prime}, \eta\right)=K_{i}\left(\psi ; \zeta+\zeta^{\prime}+\varphi ; \xi, \xi^{\prime}, \eta\right) .
$$

Then

$$
\operatorname{Ev} R_{C}\left(: K_{1}:_{\zeta, D}, \cdots,: K_{\ell} \xi_{\zeta, D}\right)(f)=: Q\left(\bar{K}_{1}, \cdots, \bar{K}_{\ell}\right)\left(f^{\prime}\right):_{\zeta, D} .
$$

(ii) Let $K\left(\psi, \zeta ; \xi, \xi^{\prime}, \eta\right)$ be a Grassmann function with $K\left(\psi, \zeta ; \xi, \xi^{\prime}, 0\right)=0$. Set

$$
\bar{K}\left(\psi ; \zeta, \zeta^{\prime}, \varphi ; \xi, \xi^{\prime}, \eta\right)=K\left(\psi ; \zeta+\zeta^{\prime}+\varphi ; \xi, \xi^{\prime}, \eta\right) .
$$

Then, when $\mathcal{R}_{: K: \zeta, D}, C$ is considered as an operator over $\mathcal{A}$,

$$
\operatorname{Ev}\left(\mathcal{R}_{: K:_{\zeta, D}, C}(f)\right)=: \mathcal{Q}_{\bar{K}}\left(f^{\prime}\right)_{\zeta, D}
$$


Proof. Applying Remark VIII.6 and Cor. A.3 to the variable $\zeta$ we see that

$$
\begin{aligned}
& \operatorname{Ev}\left(: K_{1}\left(\psi, \zeta ; \xi, \xi^{\prime}, \eta\right):_{\zeta, D} \cdots: K_{\ell}\left(\psi, \zeta ; \xi, \xi^{\prime}, \eta\right):_{\zeta, D} \cdot f(\psi, \vec{\zeta} ; \eta)\right) \\
&=\operatorname{Ev}\left(: K_{1}:_{\zeta, D} \cdots \cdot K_{\ell}:_{\zeta, D} \cdot \operatorname{Ev} f(\psi, \vec{\zeta} ; \eta)\right) \\
&=\operatorname{Ev}\left(: K_{1}:_{\zeta, D} \cdots \cdot K_{\ell} \cdots, D \cdot f^{\prime}:_{\zeta, D}\right) \\
&=: \int:\left(\prod_{i=1}^{\ell}: K_{i}\left(\psi, \zeta+\zeta^{\prime}+\varphi ; \xi, \xi^{\prime}, \eta\right):_{\zeta^{\prime}, D}\right):_{\varphi, D}: f^{\prime}(\psi, \zeta \\
&+\varphi ; \eta):_{\varphi, D} d \mu_{D}\left(\zeta^{\prime}, \varphi\right)_{{ }_{\zeta, D}} .
\end{aligned}
$$

Applying each of the following operations to both sides of this equation:

- Wick order each $: K_{i}:_{\zeta, D}$ with respect to $C$ in the $\xi^{\prime}$ variable

- Wick order the product $\prod_{i}: K_{i}: \xi^{\prime}, C$ with respect to $C$ in the $\eta$ variable

- Integrate using $\int \cdot d \mu_{C}\left(\xi^{\prime}, \eta\right)$

- Wick order the result with respect to $C$ in the $\xi$ variable

yields $\operatorname{Ev} R_{C}\left(: K_{1}:_{\zeta, D}, \cdots,: K_{\ell: \zeta, D}\right)(f)$ on the left and $: Q\left(\bar{K}_{1}, \cdots, \bar{K}_{\ell}\right)\left(f^{\prime}\right):_{\zeta, D}$ on the right.

(ii) follows from part (i), the definition of $\mathcal{Q}_{\bar{K}}$ and Remark III.6.

As in Subsect. VII.1, we define for Grassmann functions $\bar{K}_{2}\left(\psi ; \zeta, \zeta^{\prime}, \varphi ; \xi, \xi^{\prime}, \eta\right), \cdots$, $\bar{K}_{\ell}\left(\psi ; \zeta, \zeta^{\prime}, \varphi ; \xi, \xi^{\prime}, \eta\right)$ and $f(\psi, \zeta ; \xi)$ the Grassmann function $\tilde{Q}\left(\bar{K}_{2}, \cdots, \bar{K}_{\ell}\right)$ by

$$
\begin{aligned}
& \tilde{Q}\left(\bar{K}_{2}, \cdots, \bar{K}_{\ell}\right)(f)\left(\psi ; \zeta, \zeta^{\prime}, \varphi ; \xi, \xi^{\prime}, \eta\right) \\
& =\iint:\left(\prod_{i=2}^{\ell}: \bar{K}_{i}\left(\psi ; \zeta, \zeta^{\prime}+\zeta^{\prime \prime}, \varphi^{\prime} ; \xi, \xi^{\prime}+\xi^{\prime \prime}, \eta^{\prime}\right): \underset{\substack{\zeta^{\prime \prime}, D \\
\xi^{\prime \prime}, C}}{:}\right)_{\substack{\varphi^{\prime}, D \\
\eta^{\prime}, C}}^{.} \\
& : f\left(\psi, \zeta+\varphi+\varphi^{\prime} ; \eta+\eta^{\prime}\right): \varphi^{\prime}, D d \mu_{D}\left(\zeta^{\prime \prime}, \varphi^{\prime}\right) d \mu_{C}\left(\xi^{\prime \prime}, \eta^{\prime}\right) \text {. }
\end{aligned}
$$

We have, as in Prop. VII.1

Proposition VIII.13. Let $\quad \bar{K}_{1}\left(\psi ; \zeta, \zeta^{\prime}, \varphi ; \xi, \xi^{\prime}, \eta\right), \cdots, \bar{K}_{\ell}\left(\psi ; \zeta, \zeta^{\prime}, \varphi ; \xi, \xi^{\prime}, \eta\right)$ be Grassmann functions with $\bar{K}_{i}\left(\psi ; \zeta, \zeta^{\prime}, \varphi ; \xi, \xi^{\prime}, 0\right)=0$. Then for any Grassmann function $f(\psi, \zeta, \xi)$,

$$
\begin{aligned}
& Q\left(\bar{K}_{1}, \cdots, \bar{K}_{\ell}\right)(f)(\psi, \zeta ; \xi) \\
& =: \iint: \bar{K}_{1}\left(\psi ; \zeta, \zeta^{\prime}, \varphi ; \xi, \xi^{\prime}, \eta\right):_{\substack{\xi^{\prime}, \varphi ; D \\
\xi^{\prime}, \eta, C}} \\
& \quad: \tilde{Q}\left(\bar{K}_{2}, \cdots, \bar{K}_{\ell}\right)(f)\left(\psi ; \zeta, \zeta^{\prime}, \varphi ; \xi, \xi^{\prime}, \eta\right):_{\substack{\zeta^{\prime}, \varphi ; D ; C \\
\xi^{\prime}, D^{\prime}}} d \mu_{D}\left(\zeta^{\prime}, \varphi\right) d \mu_{C}\left(\xi^{\prime}, \eta\right):_{\xi, C}
\end{aligned}
$$

Lemma VIII.14. Let $\bar{K}_{i}\left(\psi ; \zeta, \zeta^{\prime}, \varphi ; \xi, \xi^{\prime}, \eta\right), i=1, \cdots, \ell$ be Grassmann functions such that $\bar{K}_{i}\left(\psi ; \zeta, \zeta^{\prime}, \varphi ; \xi, \xi^{\prime}, 0\right)=0$. Furthermore let $f(\psi, \zeta, \xi)$ be any Grassmann function. Set

$$
: f_{1}(\psi, \zeta, \xi): \xi, C=\frac{1}{\ell !} Q\left(\bar{K}_{1}, \cdots, \bar{K}_{\ell}\right)(: f: \xi, C)(\psi, \zeta, \xi)
$$


Then

$$
\begin{aligned}
& N\left(f_{1} ; \alpha\right) \leq \frac{1}{\alpha^{\ell}} N(f ; 2 \alpha) \prod_{i=1}^{\ell} N\left(\bar{K}_{i} ; \alpha\right), \\
& N\left(f_{1}(\psi, 0, \xi) ; \alpha\right) \leq \frac{1}{\alpha^{\ell}} N(f ; \alpha) \prod_{i=1}^{\ell} N\left(\bar{K}_{i} ; \alpha\right), \\
& N\left(\frac{1}{(\ell-1) !} \tilde{Q}\left(\bar{K}_{2}, \cdots, \bar{K}_{\ell}\right)(: f: \xi, C) ; \alpha\right) \leq \frac{1}{\alpha^{\ell-1}} N(f ; 4 \alpha) \prod_{i=2}^{\ell} N\left(\bar{K}_{i} ; 2 \alpha\right), \\
& N_{\mathrm{impr}}\left(\frac{1}{(\ell-1) !} \tilde{Q}\left(\bar{K}_{2}, \cdots, \bar{K}_{\ell}\right)(: f: \xi, C) ; \alpha\right) \leq \frac{1}{\alpha^{\ell-1}} N_{\mathrm{impr}}(f ; 4 \alpha) \prod_{i=2}^{\ell} N_{\mathrm{impr}}\left(\bar{K}_{i} ; 2 \alpha\right) .
\end{aligned}
$$

Proof. Set $\tilde{f}\left(\psi ; \zeta, \varphi^{\prime}, \xi\right)=f\left(\psi ; \zeta+\varphi^{\prime}, \xi\right)$ and

$$
\begin{aligned}
& : \tilde{f}_{1}\left(\psi ; \zeta, \zeta^{\prime}, \varphi, \varphi^{\prime} ; \xi\right): \xi, C=\frac{1}{\ell !}: R_{C}\left(: \bar{K}_{1}: \zeta^{\prime}, D\right. \\
& , \cdots, \bar{K}_{\ell}: \zeta^{\prime}, D \\
& : \tilde{f}_{2}\left(\psi ; \zeta, \zeta^{\prime}, \varphi, \varphi^{\prime} ; \xi\right): \xi, C=\frac{1}{\ell !} R_{C}\left(\bar{K}_{1}, \cdots, \bar{K}_{\ell}\right)(: \tilde{f}: \xi, C),
\end{aligned}
$$

where $R_{C}$ is considered as an operator over the Grassmann algebra with coefficients in $A$, generated by $\psi_{i}, \zeta_{i}, \zeta_{i}^{\prime}, \varphi_{i}, \varphi_{i}^{\prime}$. Then $f_{1}(\psi, \zeta, \xi)=\int \tilde{f}_{1}\left(\psi ; \zeta, \zeta^{\prime}, \varphi, \varphi ; \xi\right) d \mu_{D}\left(\zeta^{\prime}, \varphi\right)$, and consequently by Lemma II.31 (twice, with $C$ replaced by $D$ ), Prop. III.7 and Remark II.24,

$$
N\left(f_{1}\right) \leq N\left(\tilde{f}_{2}\right) \leq \frac{1}{\alpha^{\ell}} N(\tilde{f}) \prod_{i=1}^{\ell} N\left(\bar{K}_{i}\right) \leq \frac{1}{\alpha^{\ell}} N(f ; 2 \alpha) \prod_{i=1}^{\ell} N\left(\bar{K}_{i} ; \alpha\right) .
$$

When $\zeta$ is set to zero,

$$
\begin{aligned}
: \tilde{f}_{2}\left(\psi ; 0, \zeta^{\prime}, \varphi, \varphi^{\prime} ; \xi\right): \xi, C & =\left.\frac{1}{\ell !} R_{C}\left(\bar{K}_{1}, \cdots, \bar{K}_{\ell}\right)(: \tilde{f}: \xi, C)\right|_{\zeta=0} \\
& =\left.\frac{1}{\ell !} R_{C}\left(\bar{K}_{1}, \cdots, \bar{K}_{\ell}\right)(: f: \xi, C)\right|_{\zeta=0}
\end{aligned}
$$

so

$$
N\left(f_{1}(\psi, 0, \xi)\right) \leq N\left(\tilde{f}_{2}(\psi, 0, \xi)\right) \leq \frac{1}{\alpha^{\ell}} N(f) \prod_{i=1}^{\ell} N\left(\bar{K}_{i}\right) .
$$

The proofs of the other inequalities are similar.

\section{Overlapping Loops Created by the Second Covariance}

In this section we prove Theorem VI.10 in the general case. We assume that $A$ is a superalgebra in which all elements have degree zero and that $\|\cdot\|$ and $\|\cdot\|_{\text {impr }}$ are two systems of symmetric seminorms on the spaces $A \otimes V^{\otimes n}$ such that the covariances $(C, D)$ have improved integration constants $\mathfrak{c}, \mathrm{b}, J$ for these families of seminorms. 
IX.1. Implementing Overlapping Loops.

Proposition IX.1. Let $K\left(\psi ; \zeta, \zeta^{\prime}, \varphi ; \xi, \xi^{\prime}, \eta\right)$ be an even Grassmann function such that $K\left(\psi ; \zeta, \zeta^{\prime}, \varphi ; \xi, \xi^{\prime}, 0\right)=0$. Decompose

$$
K=K^{\prime}+K^{\prime \prime}
$$

where $K^{\prime}$ has degree at most two in the variables $\zeta^{\prime}, \xi^{\prime}, \varphi, \eta$ and $K^{\prime \prime}$ has degree at least three in these variables. Let $f(\psi ; \zeta ; \xi)$ be a Grassmann function, and set

$$
: g: \xi, C=\mathcal{Q}_{K}(: f: \xi, C), \quad: g^{\prime}: \xi, C=\mathcal{Q}_{K^{\prime}}(: f: \xi, C) .
$$

Then, if $\alpha \geq 2$ and $N(K ; 2 \alpha)_{0}<\alpha$,

$$
N_{\text {impr }}\left(g-g^{\prime} ; \alpha\right) \leq \frac{2^{5} J}{\alpha^{6}} N(f ; 4 \alpha) \frac{N(K ; 2 \alpha)}{1-\frac{1}{\alpha} N(K ; 2 \alpha)} .
$$

The proof is analogous to the proof of Prop. VII.4 and is given following

Lemma IX.2. Let $K\left(\psi ; \zeta, \zeta^{\prime}, \varphi ; \xi, \xi^{\prime}, \eta\right)$ be an even Grassmann function that satisfies $K\left(\psi ; \zeta, \zeta^{\prime}, \varphi ; \xi, \xi^{\prime}, 0\right)=0$. Decompose

$$
K=K^{\prime}+K^{\prime \prime}
$$

where $K^{\prime}$ has degree at most two in the variables $\zeta^{\prime}, \xi^{\prime}, \varphi, \eta$ and $K^{\prime \prime}$ has degree at least three in these variables. Let each of the functions $K^{(1)}, \cdots, K^{(\ell)}$ be either $K^{\prime}$ or $K^{\prime \prime}$, and assume that at least one of them is equal to $K^{\prime \prime}$. Let $f(\psi ; \zeta ; \xi)$ be a Grassmann function, and set

$$
\frac{1}{\ell !} Q\left(K^{(1)}, \cdots, K^{(\ell)}\right)(: f:)(\psi ; \zeta ; \xi)=: f^{\prime}(\psi ; \zeta ; \xi): \xi, C,
$$

where $: f:$ is shorthand for $: f(\psi ; \zeta ; \xi): \xi, C$. Then, if $\alpha \geq 2$,

$$
N_{\text {impr }}\left(f^{\prime} ; \alpha\right) \leq \frac{2^{5}}{\ell \alpha^{\ell+5}} J N(f ; 4 \alpha) N(K ; 2 \alpha)^{\ell} .
$$

Proof. We may assume that $K^{(1)}=K^{\prime \prime}$. Set

$$
g\left(\psi ; \zeta, \zeta^{\prime}, \varphi ; \xi, \xi^{\prime}, \eta\right)=\tilde{Q}\left(K^{(2)}, \cdots, K^{(\ell)}\right)(: f:) .
$$

By Lemma VIII.14

$$
\frac{1}{(\ell-1) !} N(g) \leq \frac{1}{\alpha^{\ell-1}} N(f ; 4 \alpha) N(K ; 2 \alpha)^{\ell-1} .
$$

By Prop. VIII.13,

$$
\begin{aligned}
& f^{\prime}(\psi ; \zeta ; \xi)=\frac{1}{\ell !} \iint: K^{\prime \prime}\left(\psi ; \zeta, \zeta^{\prime}, \varphi ; \xi, \xi^{\prime}, \eta\right):_{\substack{\zeta^{\prime}, \varphi ; D \\
\xi^{\prime}, \eta \rightarrow C}}
\end{aligned}
$$

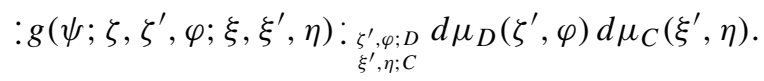

Proposition VI.4 implies that

$$
\begin{aligned}
N_{\mathrm{impr}}\left(f^{\prime}\right) & \leq \frac{27 J}{\ell ! \alpha^{6}} N\left(K^{\prime \prime}\right) N(g) \\
& \leq \frac{2^{5}}{\ell \alpha^{\ell+5}} J N(f ; 4 \alpha) N(K ; 2 \alpha)^{\ell} .
\end{aligned}
$$


Proof of Prop. IX.1. By Def. VIII.1 ii

$$
g=\sum_{\ell=1}^{\infty} g_{\ell}, \quad \quad g^{\prime}=\sum_{\ell=1}^{\infty} g_{\ell}^{\prime}
$$

where

$$
\begin{aligned}
& : g_{\ell}:=\frac{1}{\ell !} Q(K, \cdots, K)(: f:) \\
& : g_{\ell}^{\prime}:=\frac{1}{\ell !} Q\left(K^{\prime}, \cdots, K^{\prime}\right)(: f:)
\end{aligned}
$$

Since

$$
\begin{aligned}
Q( & (K, \cdots, K)-Q\left(K^{\prime}, \cdots, K^{\prime}\right) \\
= & Q\left(K-K^{\prime}, K, \cdots, K\right)+Q\left(K^{\prime}, K-K^{\prime}, K, \cdots, K\right) \\
& +\cdots+Q\left(K^{\prime}, \cdots, K-K^{\prime}\right) \\
= & Q\left(K^{\prime \prime}, K, \cdots, K\right)+Q\left(K^{\prime}, K^{\prime \prime}, K, \cdots, K\right)+\cdots+Q\left(K^{\prime}, \cdots, K^{\prime \prime}\right),
\end{aligned}
$$

it follows from Lemma IX.2 that

$$
N_{\text {impr }}\left(g_{\ell}-g_{\ell}^{\prime}\right) \leq \frac{2^{5}}{\alpha^{\ell+5}} J N(f ; 4 \alpha) N(K ; 2 \alpha)^{\ell} .
$$

Therefore

$$
N_{\mathrm{impr}}\left(g-g^{\prime}\right) \leq \sum_{\ell=1}^{\infty} N_{\mathrm{impr}}\left(g_{\ell}-g_{\ell}^{\prime}\right) \leq \frac{2^{5} J}{\alpha^{6}} N(f ; 4 \alpha) \frac{N(K ; 2 \alpha)}{1-\frac{1}{\alpha} N(K ; 2 \alpha)} .
$$

Proposition IX.3. Let $K\left(\psi ; \zeta, \zeta^{\prime}, \varphi ; \xi, \xi^{\prime}, \eta\right)$ be an even Grassmann function of degree at most two in the variables $\zeta^{\prime}, \xi^{\prime}, \varphi, \eta$ and of degree at least one in $\eta$. Write

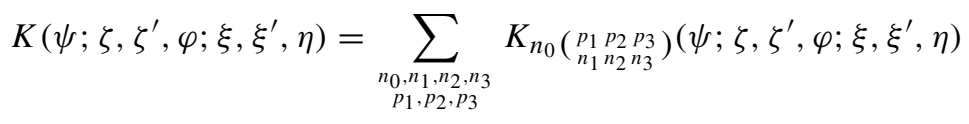

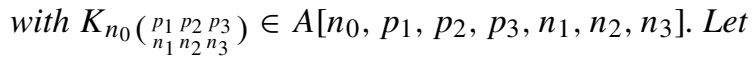

$$
K_{\cdot, 1}=\sum_{n_{0}, n_{1}, p_{1}} K_{n_{0}}\left(\begin{array}{l}
p_{1} 00 \\
n_{1} 01
\end{array}\right)
$$

be the part of $K$ that has degree precisely one in $\zeta^{\prime}, \xi^{\prime}, \varphi, \eta$.

Furthermore let $T(\psi ; \varphi ; \eta)$ be a Grassmann function that has degree two in the variables $\varphi, \eta$ and degree at least one in the variable $\eta$. Write $T=T_{11}+T_{02}$, where $T_{11}$ has degree one in $\varphi$ and $\eta$, and $T_{02}$ has degree two in $\eta$. Set

$$
\begin{aligned}
T_{\text {mix }}\left(\psi ; \varphi^{\prime}, \varphi^{\prime \prime} ; \eta^{\prime}, \eta^{\prime \prime}\right)= & T_{11}\left(\psi ; \varphi^{\prime} ; \eta^{\prime \prime}\right)+T_{11}\left(\psi ; \varphi^{\prime \prime} ; \eta^{\prime}\right) \\
& +\left[T_{02}\left(\psi ; 0 ; \eta^{\prime}+\eta^{\prime \prime}\right)-T_{02}\left(\psi ; 0 ; \eta^{\prime}\right)-T_{02}\left(\psi ; 0 ; \eta^{\prime \prime}\right)\right]
\end{aligned}
$$


Furthermore set

$$
\begin{aligned}
\tilde{K}_{1}\left(\psi ; \zeta, \zeta^{\prime}, \varphi ; \xi, \xi^{\prime}, \eta\right)= & \iint: T\left(\psi ; \varphi^{\prime} ; \eta^{\prime}\right):_{\eta^{\prime}, C}: K(\psi ; \zeta \\
& \left.+\varphi^{\prime}, \zeta^{\prime}, \varphi ; \xi+\eta^{\prime}, \xi^{\prime}, \eta\right): \varphi_{\varphi^{\prime}, D} d \mu_{D}\left(\varphi^{\prime}\right) d \mu_{C}\left(\eta^{\prime}\right),
\end{aligned}
$$
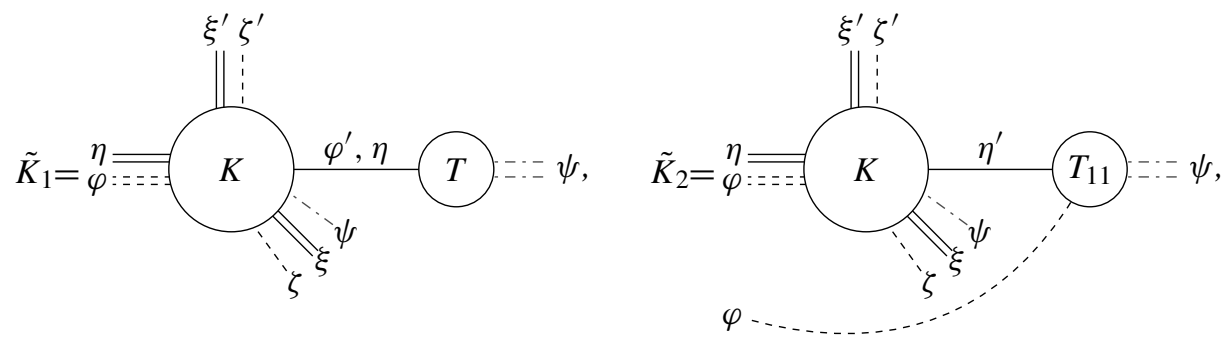

and $\tilde{K}=\tilde{K}_{1}+\tilde{K}_{2}$,

$$
\begin{aligned}
\hat{K}(\psi ; \zeta, \varphi ; \xi, \eta)= & \iint T_{\operatorname{mix}}\left(\psi ; \varphi^{\prime}, \varphi^{\prime \prime} ; \eta^{\prime}, \eta^{\prime \prime}\right): K_{,, 1}(\psi ; \zeta \\
& \left.+\varphi^{\prime}, \zeta^{\prime}, \varphi ; \xi+\eta^{\prime}, \xi^{\prime}, \eta\right):{ }_{\varphi^{\prime}, D} \\
& : K_{,, 1}\left(\psi ; \zeta+\varphi^{\prime \prime}, \zeta^{\prime}, \varphi ; \xi+\eta^{\prime \prime}, \xi^{\prime}, \eta\right): \underset{\varphi^{\prime \prime}, D}{\eta^{\prime \prime}, C} \\
& \times d \mu_{D}\left(\zeta^{\prime}, \varphi^{\prime}, \varphi^{\prime \prime}\right) d \mu_{C}\left(\xi^{\prime}, \eta^{\prime}, \eta^{\prime \prime}\right),
\end{aligned}
$$

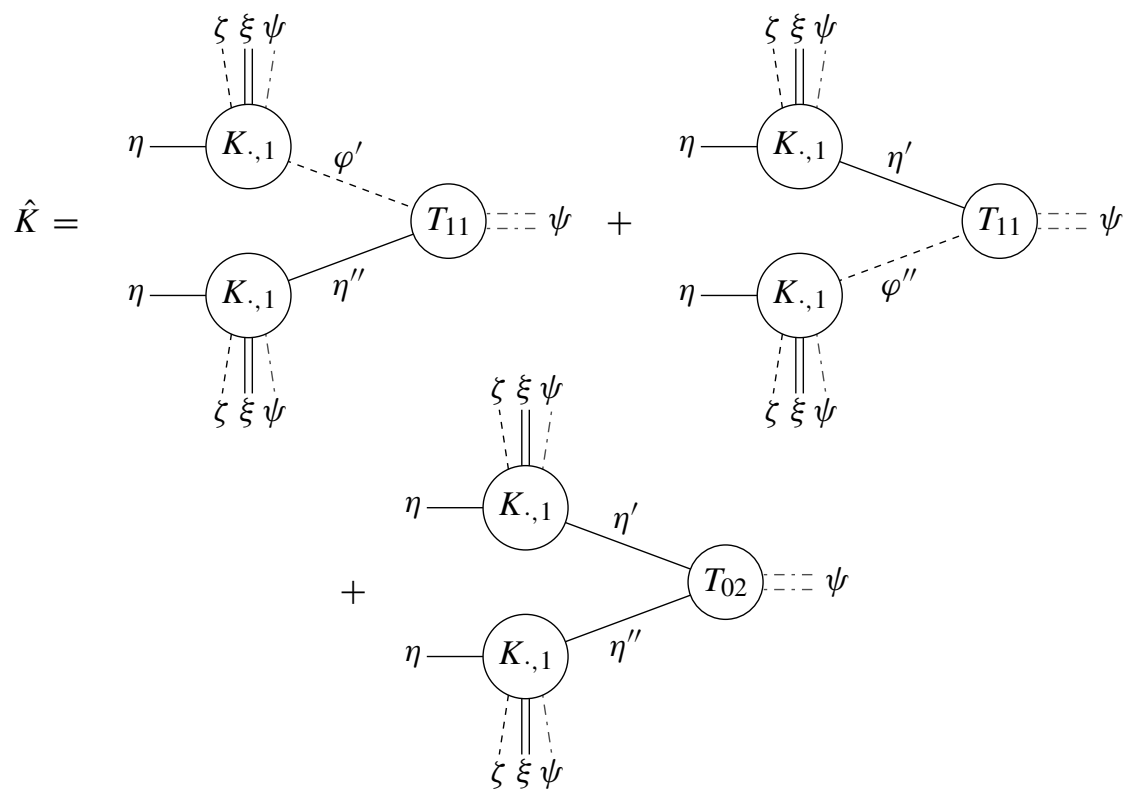


Finally let $f(\psi ; \zeta ; \xi)$ be a Grassmann function and set

$$
\begin{aligned}
f^{\prime}(\psi ; \zeta, \varphi ; \xi) & =\tilde{Q}(T) Q(K, K)(: f: \xi, C)(\psi ; \zeta, 0, \varphi ; 0,0, \xi), \\
: \tilde{f}(\psi ; \zeta, \varphi ; \xi): \xi, C & =2 Q(\tilde{K}, K)(: f: \xi, C)(\psi ; \zeta+\varphi ; \xi), \\
: \hat{f}(\psi ; \zeta, \varphi ; \xi): \xi, C & =Q(\hat{K})(: f: \xi, C)(\psi ; \zeta+\varphi ; \xi) .
\end{aligned}
$$

Then, if $\alpha \geq 2$,

$$
N_{\text {impr }}\left(f^{\prime}-(\tilde{f}+\hat{f}) ; \alpha\right) \leq \frac{2^{9} J}{\alpha^{10}} N(f ; 4 \alpha) N(K ; 4 \alpha)^{2} N(T ; 2 \alpha) .
$$

The proof is similar to the proof of Prop. VII.7 and is given following

Lemma IX.4. Let $B\left(\psi ; \varphi^{\prime}, \varphi^{\prime \prime} ; \eta^{\prime}, \eta^{\prime \prime}\right)$ be a Grassmann function that has degree one in the variables $\varphi^{\prime}, \eta^{\prime}$, degree one in the variables $\varphi^{\prime \prime}, \eta^{\prime \prime}$ and degree at least one in the variables $\eta^{\prime}, \eta^{\prime \prime}$. Furthermore let $H\left(\psi ; \zeta, \zeta^{\prime}, \gamma ; \xi, \xi^{\prime}, \eta\right), K\left(\psi ; \zeta, \zeta^{\prime}, \gamma ; \xi, \xi^{\prime}, \eta\right)$ be even Grassmann functions that vanish for $\eta=0$. Assume that $H$ or $K$ has degree at least two in the variables $\zeta^{\prime}, \gamma, \xi^{\prime}, \eta$. Let $f(\psi ; \zeta ; \xi)$ be any Grassmann function and set

$$
\begin{aligned}
& g(\psi ; \zeta, \varphi ; \xi)=\iint:\left[\iint B\left(\psi ; \varphi^{\prime}, \varphi^{\prime \prime} ; \eta^{\prime}, \eta^{\prime \prime}\right): H(\psi ; \zeta+\varphi\right.
\end{aligned}
$$

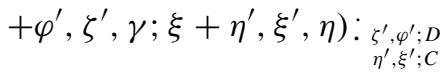

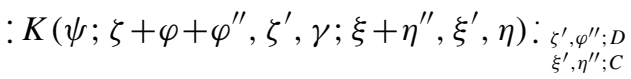

$$
\begin{aligned}
& \left.\times d \mu_{D}\left(\varphi^{\prime}, \varphi^{\prime \prime}, \zeta^{\prime}\right) d \mu_{C}\left(\eta^{\prime}, \eta^{\prime \prime}, \xi^{\prime}\right)\right] \cdot \underset{\eta, C}{\gamma, D} \\
& : f(\psi ; \zeta+\varphi+\gamma ; \eta): \underset{\eta, C}{\gamma, D} d \mu_{D}(\gamma) d \mu_{C}(\eta) \text {. }
\end{aligned}
$$

Then, if $\alpha \geq 2$,

$$
N_{\text {impr }}(g ; \alpha) \leq \frac{2^{5} J}{\alpha^{10}} N(B ; \alpha) N(H ; 4 \alpha) N(K ; 4 \alpha) N(f ; 4 \alpha) .
$$

Proof. In the proof we suppress the variable $\psi$. We assume that $K$ has degree at least two in the variables $\zeta^{\prime}, \gamma, \xi^{\prime}, \eta$. First we discuss the case that $B$ has degree one in the variable $\eta^{\prime}$. Set

$$
\begin{aligned}
& h\left(\zeta, \varphi, \gamma, \zeta^{\prime}, \varphi^{\prime \prime} ; \xi, \eta, \xi^{\prime}, \eta^{\prime \prime}\right) \\
& \quad=\iint B\left(\varphi^{\prime}, \varphi^{\prime \prime} ; \eta^{\prime}, \eta^{\prime \prime}\right): H\left(\zeta+\varphi+\varphi^{\prime}, \zeta^{\prime}, \zeta^{\prime \prime} ; \xi+\eta^{\prime}, \xi^{\prime}, \xi^{\prime \prime}\right):{\substack{\varphi^{\prime}, \prime^{\prime \prime} ; D \\
\eta^{\prime \prime}, \xi^{\prime \prime} ; C}} \cdot f\left(\zeta+\varphi+\gamma+\zeta^{\prime \prime} ; \eta+\xi^{\prime \prime}\right): \underset{\substack{\zeta^{\prime \prime}, D \\
\xi^{\prime \prime}, C}}{ } d \mu_{D}\left(\varphi^{\prime}, \zeta^{\prime \prime}\right) d \mu_{C}\left(\eta^{\prime}, \xi^{\prime \prime}\right) .
\end{aligned}
$$

By Lemma A.5, twice

$$
\begin{aligned}
& \int \int: H(\cdots, \gamma \cdots, \eta) K(\cdots, \gamma \cdots, \eta) \underset{\substack{\gamma, D \\
\eta, C}}{ }: f(\cdots, \gamma ; \eta) \underset{\substack{\gamma, D \\
\eta, C}}{ } d \mu_{C}(\eta) d \mu_{D}(\gamma) \\
&= \iint: H\left(\cdots, \zeta^{\prime \prime} \cdots, \xi^{\prime \prime}\right)_{\substack{\zeta^{\prime \prime}, D \\
\xi^{\prime \prime}, C}}: K(\cdots, \gamma \cdots, \eta)_{\substack{\gamma, D \\
\eta, C}}: f\left(\cdots, \gamma+\zeta^{\prime \prime} ; \eta+\xi^{\prime \prime}\right) \underset{\substack{\gamma, \zeta^{\prime \prime} ; D \\
\eta, \xi^{\prime \prime} ; C}}{(\text { IX) }} \\
& \quad \times d \mu_{C}\left(\eta, \xi^{\prime \prime}\right) d \mu_{D}\left(\gamma, \zeta^{\prime \prime}\right)
\end{aligned}
$$


so

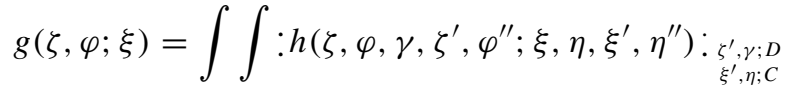

$$
\begin{aligned}
& : K\left(\zeta+\varphi+\varphi^{\prime \prime}, \zeta^{\prime}, \gamma ; \xi+\eta^{\prime \prime}, \xi^{\prime}, \eta\right): \underset{\varphi^{\prime \prime}, \zeta^{\prime}, \gamma ; D}{\eta^{\prime \prime}, \xi^{\prime}, \eta ; C} \\
& \times d \mu_{D}\left(\gamma, \varphi^{\prime \prime}, \zeta^{\prime}\right) d \mu_{C}\left(\eta, \eta^{\prime \prime}, \xi^{\prime}\right) .
\end{aligned}
$$

By iterated application of Lemma II.31 combined with Prop. II.33, first integrating $\int \cdot d \mu_{C}\left(\eta^{\prime}\right) d \mu_{D}\left(\varphi^{\prime}\right)$ and then integrating $\int \cdot d \mu_{C}\left(\xi^{\prime \prime}\right) d \mu_{D}\left(\zeta^{\prime \prime}\right)$, and Remark II.24, several times,

$$
\begin{aligned}
N(h) & \leq \frac{1}{\alpha^{4}} N(B) N\left(H\left(\zeta+\varphi+\varphi^{\prime}, \zeta^{\prime}, \zeta^{\prime \prime} ; \xi+\eta^{\prime}, \xi^{\prime}, \xi^{\prime \prime}\right) ; \alpha\right) N\left(f\left(\zeta+\varphi+\gamma+\zeta^{\prime \prime} ; \eta+\xi^{\prime \prime}\right) ; \alpha\right) \\
& \leq \frac{1}{\alpha^{4}} N(B) N\left(H\left(\zeta+\varphi, \zeta^{\prime}, \zeta^{\prime \prime} ; \xi, \xi^{\prime}, \xi^{\prime \prime}\right) ; 2 \alpha\right) N(f(\zeta+\gamma ; \eta) ; 2 \alpha) \\
& \leq \frac{1}{\alpha^{4}} N(B) N(H ; 4 \alpha) N(f ; 4 \alpha) .
\end{aligned}
$$

Since $h$, through $B$, has degree one in $\varphi^{\prime \prime}, \eta^{\prime \prime}$, only the part of $K\left(\zeta+\varphi+\varphi^{\prime \prime}, \zeta^{\prime}, \gamma ; \xi+\right.$ $\left.\eta^{\prime \prime}, \xi^{\prime}, \eta\right)$ that has degree at least one in $\varphi^{\prime \prime}, \eta^{\prime \prime}$ can contribute to $g(\zeta, \varphi ; \xi)$. As $K$ also has degree at least two in the variables $\zeta^{\prime}, \gamma, \xi^{\prime}, \eta$ and degree at least one in $\eta$, Prop. VI.4 implies that

$$
\begin{aligned}
N_{\text {impr }}(g) & \leq \frac{27 J}{\alpha^{6}} N(h) N(K ; 4 \alpha) \\
& \leq \frac{2^{5} J}{\alpha^{10}} N(B) N(H ; 4 \alpha) N(f ; 4 \alpha) N(K ; 4 \alpha) .
\end{aligned}
$$

Next we discuss the situation that $B$ has degree zero in the variable $\eta^{\prime}$. This implies that $B$ has degree one in $\varphi^{\prime}$ and degree one in $\eta^{\prime \prime}$. Set

$$
\begin{aligned}
& h\left(\zeta, \varphi, \gamma, \zeta^{\prime}, \varphi^{\prime} ; \xi, \xi^{\prime}, \eta, \eta^{\prime}\right)=\iint: H\left(\zeta+\varphi+\varphi^{\prime}, \zeta^{\prime}, \zeta^{\prime \prime} ; \xi+\eta^{\prime}, \xi^{\prime}, \xi^{\prime \prime}\right): \underset{\zeta^{\prime \prime} ; D}{\xi^{\prime \prime} ; C} \\
& : f\left(\zeta+\varphi+\gamma+\zeta^{\prime \prime} ; \eta+\xi^{\prime \prime}\right): \underset{\substack{\zeta^{\prime \prime} ; D \\
\xi^{\prime \prime} ; C}}{ } d \mu_{D}\left(\zeta^{\prime \prime}\right) d \mu_{C}\left(\xi^{\prime \prime}\right) \\
& k\left(\zeta, \varphi, \gamma, \zeta^{\prime}, \varphi^{\prime} ; \xi, \xi^{\prime}, \eta, \eta^{\prime}\right)=\iint B\left(\varphi^{\prime}, \varphi^{\prime \prime} ; \eta^{\prime}, \eta^{\prime \prime}\right): K\left(\zeta+\varphi+\varphi^{\prime \prime}, \zeta^{\prime}, \gamma ; \xi\right. \\
& \left.+\eta^{\prime \prime}, \xi^{\prime}, \eta\right): \underset{\substack{\varphi^{\prime \prime} ; D \\
\eta^{\prime \prime} ; C}}{ } d \mu_{D}\left(\varphi^{\prime \prime}\right) d \mu_{C}\left(\eta^{\prime \prime}\right) \text {. }
\end{aligned}
$$

Again by (IX.1),

$$
\begin{aligned}
g= & \iint: h\left(\zeta, \varphi, \gamma, \zeta^{\prime}, \varphi^{\prime} ; \xi, \xi^{\prime}, \eta, \eta^{\prime}\right): \underset{\substack{\varphi^{\prime}, \zeta^{\prime}, \gamma ; D \\
\eta^{\prime}, \xi^{\prime}, \eta ; C}}{ } \\
& : k\left(\zeta, \varphi, \gamma, \zeta^{\prime}, \varphi^{\prime} ; \xi, \xi^{\prime}, \eta, \eta^{\prime}\right): \underset{\substack{\varphi^{\prime}, \zeta^{\prime}, \gamma ; D \\
\eta^{\prime}, \xi^{\prime}, \eta ; C}}{ } d \mu_{D}\left(\varphi^{\prime}, \zeta^{\prime}, \gamma\right) d \mu_{C}\left(\eta^{\prime}, \xi^{\prime}, \eta\right) .
\end{aligned}
$$

By Lemma II.31, Prop. II.33 and Remark II.24,

$$
\begin{aligned}
& N(h) \leq \frac{1}{\alpha^{2}} N(H ; 4 \alpha) N(f ; 4 \alpha), \\
& N(k) \leq \frac{1}{\alpha^{2}} N(B ; \alpha) N(K ; 4 \alpha) .
\end{aligned}
$$

Since $k$ has degree at least one in $\eta$ and degree at least three in $\varphi^{\prime}, \zeta^{\prime}, \gamma, \eta^{\prime}, \xi^{\prime}, \eta$ we have

$$
N_{\text {impr }}(g) \leq \frac{27 J}{\alpha^{6}} N(h) N(k) \leq \frac{2^{5} J}{\alpha^{10}} N(H ; 4 \alpha) N(f ; 4 \alpha) N(K ; 4 \alpha) N(B) .
$$


Proof of Prop. IX.3. We again suppress $\psi$ in the proof. By definition

$$
\begin{aligned}
f^{\prime}(\zeta, \varphi ; \eta)= & \iint: T\left(\varphi^{\prime}, \eta^{\prime}\right): \eta_{\eta^{\prime}, C}: Q(K, K)(: f: \xi, C)\left(\zeta+\varphi+\varphi^{\prime} ; \eta+\eta^{\prime}\right): \varphi_{\varphi^{\prime}, D} \\
& \times d \mu_{D}\left(\varphi^{\prime}\right) d \mu_{C}\left(\eta^{\prime}\right)
\end{aligned}
$$

so that, by Lemma A.7, recalling that $T\left(\varphi^{\prime}, \eta^{\prime}\right)$ is of degree two in $\varphi^{\prime}, \eta^{\prime}$,

$$
\begin{aligned}
& f^{\prime}(\zeta, \varphi ; \xi)=\iint: T\left(\varphi^{\prime}, \eta^{\prime}\right): \eta^{\prime}, C: Q(K, K)(: f:)\left(\zeta+\varphi+\varphi^{\prime} ; \xi+\eta^{\prime}\right): \varphi_{\varphi^{\prime}, D} \\
& \times d \mu_{D}\left(\varphi^{\prime}\right) d \mu_{C}\left(\eta^{\prime}\right) \\
& =\iint: T\left(\varphi^{\prime}, \eta^{\prime}\right): \eta_{\eta^{\prime}, C}:\left[:\left(: K\left(\zeta+\varphi+\varphi^{\prime}, \zeta^{\prime}, \gamma ; \xi+\eta^{\prime}, \xi^{\prime}, \eta\right):_{\substack{\zeta^{\prime}, D \\
\xi^{\prime}, C}}\right.\right. \\
& : K\left(\zeta+\varphi+\varphi^{\prime}, \zeta^{\prime}, \gamma ; \xi+\eta^{\prime}, \xi^{\prime}, \eta\right): \underset{\substack{\zeta^{\prime}, D \\
\xi^{\prime}, C}}{\underset{\substack{\gamma, D \\
\eta, C}}{ }} \\
& \left.: f\left(\zeta+\varphi+\varphi^{\prime}+\gamma ; \eta\right): \underset{\eta, D}{\gamma, D}\right]: \underset{\eta^{\prime}, C}{: \varphi^{\prime}, D} d \mu_{D}\left(\zeta^{\prime}, \varphi^{\prime}, \gamma\right) d \mu_{C}\left(\xi^{\prime}, \eta, \eta^{\prime}\right)
\end{aligned}
$$

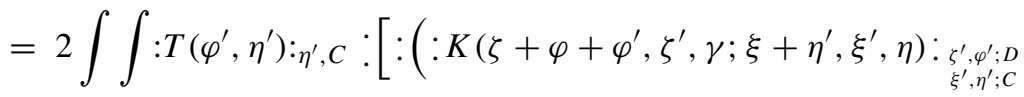

$$
\begin{aligned}
& \left.: K\left(\zeta+\varphi, \zeta^{\prime}, \gamma ; \xi, \xi^{\prime}, \eta\right): \underset{\xi^{\prime}, C}{:}\right): \underset{\gamma, D}{\gamma, D}
\end{aligned}
$$

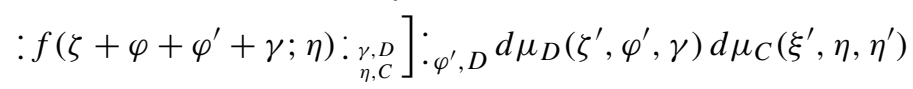

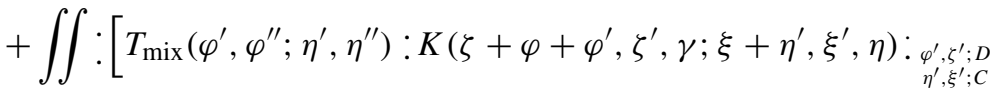

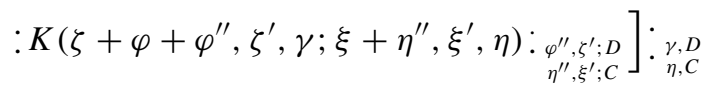

$$
\begin{aligned}
& : f(\zeta+\varphi+\gamma ; \eta): \underset{\substack{\gamma, D \\
\eta, C}}{ } d \mu_{D}\left(\varphi^{\prime}, \varphi^{\prime \prime}, \zeta^{\prime}, \gamma\right) d \mu_{C}\left(\eta^{\prime}, \eta^{\prime \prime}, \xi^{\prime}, \eta\right) . \text { (IX.2) }
\end{aligned}
$$

The $T_{\text {mix }}$ term in (IX.2) above differs from

$$
\iint: \hat{K}(\zeta+\varphi, \gamma ; \xi, \eta): \underset{\gamma, D}{\gamma, D}: f(\zeta+\varphi+\gamma ; \eta) \underset{\substack{\gamma, D \\ \eta, C}}{ } d \mu_{D}(\gamma) d \mu_{C}(\eta)=\hat{f}(\zeta, \varphi ; \xi)
$$

by

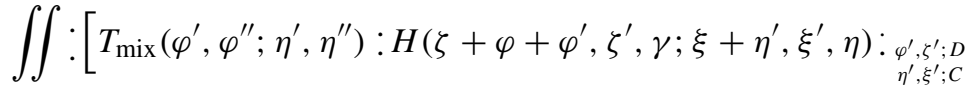

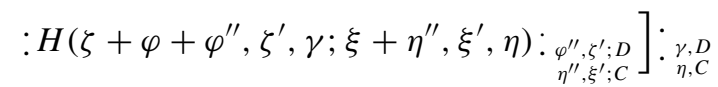

$$
\begin{aligned}
& : f(\zeta+\varphi+\gamma ; \eta) \underset{\substack{\gamma, D \\
\eta, D}}{ } d \mu_{D}\left(\varphi^{\prime}, \varphi^{\prime \prime}, \zeta^{\prime}, \gamma\right) d \mu_{C}\left(\eta^{\prime}, \eta^{\prime \prime}, \xi^{\prime}, \eta\right) \\
& +2 \iint:\left[T_{\operatorname{mix}}\left(\varphi^{\prime}, \varphi^{\prime \prime} ; \eta^{\prime}, \eta^{\prime \prime}\right): H\left(\zeta+\varphi+\varphi^{\prime}, \zeta^{\prime}, \gamma ; \xi+\eta^{\prime}, \xi^{\prime}, \eta\right): \underset{\substack{\varphi^{\prime}, \zeta^{\prime} ; D \\
\eta^{\prime}, \xi^{\prime} ; C}}{ }\right. \\
& \left.: K \cdot, 1\left(\zeta+\varphi+\varphi^{\prime \prime}, \zeta^{\prime}, \gamma ; \xi+\eta^{\prime \prime}, \xi^{\prime}, \eta\right): \underset{\substack{\varphi^{\prime \prime}, \zeta^{\prime} ; D \\
\eta^{\prime \prime}, \xi^{\prime} ; C}}{:}\right] \cdot \begin{array}{l}
\gamma, D \\
\eta, C
\end{array} \\
& : f(\zeta+\varphi+\gamma ; \eta): \underset{\gamma, C}{\gamma, D} d \mu_{D}\left(\varphi^{\prime}, \varphi^{\prime \prime}, \zeta^{\prime}, \gamma\right) d \mu_{C}\left(\eta^{\prime}, \eta^{\prime \prime}, \xi^{\prime}, \eta\right),
\end{aligned}
$$


where $K=K_{\cdot, 1}+H$. By Lemma IX.4, the improved norm of this difference is bounded by

$$
\begin{aligned}
& 3 \frac{2^{5} J}{\alpha^{10}} N\left(T_{\mathrm{mix}} ; \alpha\right) N(H ; 4 \alpha) N(K ; 4 \alpha) N(f ; 4 \alpha) \\
& \leq \frac{2^{9} J}{\alpha^{10}} N(T ; 2 \alpha) N(K ; 4 \alpha)^{2} N(f ; 4 \alpha) .
\end{aligned}
$$

We apply Lemma A.5 to the variable $\varphi^{\prime}$ in the $2: T\left(\varphi^{\prime}, \eta^{\prime}\right):_{\eta^{\prime}, C}$ term of (IX.2). We get

$$
\begin{aligned}
& 2 \iint: T\left(\varphi^{\prime}+\varphi^{\prime \prime} ; \eta^{\prime}\right): \eta^{\prime}, C:\left(: K\left(\zeta+\varphi+\varphi^{\prime}, \zeta^{\prime}, \gamma ; \xi+\eta^{\prime}, \xi^{\prime}, \eta\right):_{\substack{\zeta^{\prime}, \varphi^{\prime} ; D \\
\xi^{\prime}, \eta^{\prime} ; C}}\right. \\
& \left.: K\left(\zeta+\varphi, \zeta^{\prime}, \gamma ; \xi, \xi^{\prime}, \eta\right): \underset{\xi^{\prime}, C}{\underset{\xi^{\prime}, D}{ }}\right): \underset{\eta, C}{\gamma, D}
\end{aligned}
$$

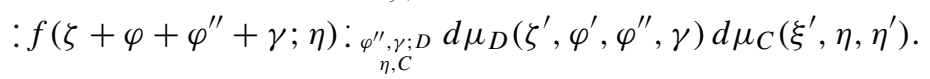

As $T$ has degree at most one in $\varphi^{\prime}+\varphi^{\prime \prime}$, this is equal to

$$
\begin{aligned}
& 2 \iint:\left(: T\left(\varphi^{\prime} ; \eta^{\prime}\right): \eta^{\prime}, C: K\left(\zeta+\varphi+\varphi^{\prime}, \zeta^{\prime}, \gamma ; \xi+\eta^{\prime}, \xi^{\prime}, \eta\right):_{\substack{\zeta^{\prime}, \varphi^{\prime} ; D \\
\xi^{\prime}, \eta^{\prime} ; C}}^{:}\right. \\
& \left.: K\left(\zeta+\varphi, \zeta^{\prime}, \gamma ; \xi, \xi^{\prime}, \eta\right): \underset{\xi_{\xi^{\prime}, C}^{\prime}}{\underset{\zeta^{\prime}, D}{\gamma, C}}\right): \underset{\gamma, D}{\gamma, \zeta^{\prime}}
\end{aligned}
$$

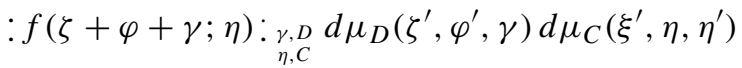

$$
\begin{aligned}
& +2 \iint: T_{11}\left(\varphi^{\prime \prime} ; \eta^{\prime}\right): \eta^{\prime}, C:\left(: K\left(\zeta+\varphi, \zeta^{\prime}, \gamma ; \xi+\eta^{\prime}, \xi^{\prime}, \eta\right): \underset{\substack{\zeta^{\prime}, D \\
\xi^{\prime}, \eta^{\prime} ; C}}{:}\right. \\
& \left.: K\left(\zeta+\varphi, \zeta^{\prime}, \gamma ; \xi, \xi^{\prime}, \eta\right): \underset{\substack{\zeta^{\prime}, D \\
\xi^{\prime}, C}}{:}\right): \underset{\eta, D}{\gamma, D} \\
& : f\left(\zeta+\varphi+\varphi^{\prime \prime}+\gamma ; \eta\right): \underset{\varphi^{\prime \prime}, \gamma ; D}{\eta, C} d \mu_{D}\left(\zeta^{\prime}, \varphi^{\prime \prime}, \gamma\right) d \mu_{C}\left(\xi^{\prime}, \eta, \eta^{\prime}\right) .
\end{aligned}
$$

Applying Lemma A.5, with $\xi$ replaced by $\gamma$ and $\xi^{\prime}$ replaced by $\varphi^{\prime \prime}$, to the second term, this is equal to

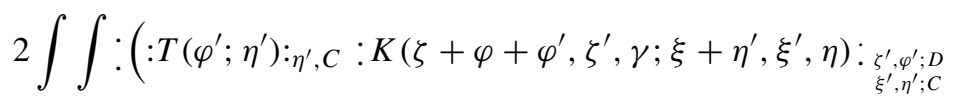

$$
\begin{aligned}
& : K\left(\zeta+\varphi, \zeta^{\prime}, \gamma ; \xi, \xi^{\prime}, \eta\right): \underset{\substack{\zeta^{\prime}, D \\
\xi^{\prime}, D}}{\underset{\gamma, C}{\eta, D}}
\end{aligned}
$$

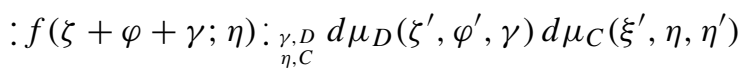

$$
\begin{aligned}
& +2 \iint:\left(T_{11}\left(\gamma ; \eta^{\prime}\right): K\left(\zeta+\varphi, \zeta^{\prime}, \gamma ; \xi+\eta^{\prime}, \xi^{\prime}, \eta\right): \underset{\substack{\zeta^{\prime}, D \\
\xi^{\prime}, \eta^{\prime} ; C}}{:}\right. \\
& \left.: K\left(\zeta+\varphi, \zeta^{\prime}, \gamma ; \xi, \xi^{\prime}, \eta\right): \underset{\xi^{\prime}, D}{\xi^{\prime}, D}\right): \underset{\gamma, C}{\gamma, D} \\
& : f(\zeta+\varphi+\gamma ; \eta): \underset{\substack{\gamma ; D \\
\eta, C}}{ } d \mu_{D}\left(\zeta^{\prime}, \gamma\right) d \mu_{C}\left(\xi^{\prime}, \eta, \eta^{\prime}\right)
\end{aligned}
$$

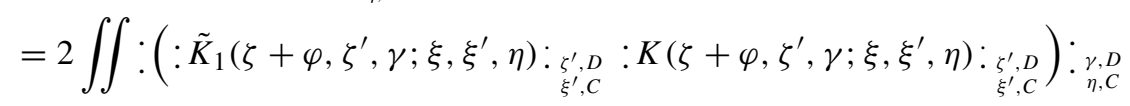

$$
\begin{aligned}
& : f(\zeta+\varphi+\gamma ; \eta): \underset{\substack{\gamma, D \\
\eta, C}}{ } d \mu_{D}\left(\zeta^{\prime}, \gamma\right) d \mu_{C}\left(\xi^{\prime}, \eta\right)
\end{aligned}
$$




$$
\begin{aligned}
& +2 \iint:\left(: \tilde{K}_{2}\left(\zeta+\varphi, \zeta^{\prime}, \gamma ; \xi, \xi^{\prime}, \eta\right):_{\zeta_{\xi^{\prime}, C}, D}: K\left(\zeta+\varphi, \zeta^{\prime}, \gamma ; \xi, \xi^{\prime}, \eta\right):_{\substack{\zeta^{\prime}, D \\
\xi^{\prime}, C}}\right):{ }_{\eta, C}^{\gamma, D} \\
& : f(\zeta+\varphi+\gamma ; \eta): \underset{\eta, D}{\gamma, C} d \mu_{D}\left(\zeta^{\prime}, \gamma\right) d \mu_{C}\left(\xi^{\prime}, \eta\right) .
\end{aligned}
$$

So the $2: T\left(\varphi^{\prime}, \eta^{\prime}\right): \eta^{\prime}, C$ term of (IX.2) equals

$$
\tilde{f}_{1}(\zeta, \varphi ; \xi)+\tilde{f}_{2}(\zeta, \varphi ; \xi)
$$

where

$$
\begin{aligned}
& : \tilde{f}_{1}(\zeta, \varphi ; \xi): \xi, C=2 Q\left(\tilde{K}_{1}, K\right)(: f:)(\zeta+\varphi ; \xi) \\
& : \tilde{f}_{2}(\zeta, \varphi ; \xi): \xi, C=2 Q\left(\tilde{K}_{2}, K\right)(: f:)(\zeta+\varphi ; \xi) .
\end{aligned}
$$

As $\tilde{f}=\tilde{f}_{1}+\tilde{f}_{2}$, the proposition follows.

IX.2. Tails. In this subsection let $K\left(\psi ; \zeta, \zeta^{\prime}, \varphi ; \xi, \xi^{\prime}, \eta\right)$ be an even Grassmann function that has degree at least four in the variables $\psi, \zeta, \zeta^{\prime}, \varphi, \xi, \xi^{\prime}, \eta$ such that $K\left(\psi ; \zeta, \zeta^{\prime}, \varphi\right.$; $\left.\xi, \xi^{\prime}, 0\right)=0$. We always write

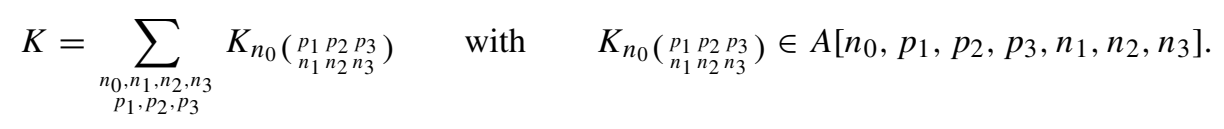

Definition IX.5. (i) An n-legged tail with at least e external legs is a Grassmann function

$$
T(\psi ; \varphi ; \eta) \in \bigoplus_{d \geq e} \bigoplus_{\substack{n_{1}+n_{2}=n \\ n_{2} \geq 1}} A\left[d, n_{1}, n_{2}\right]
$$

A n-legged tail is a n-legged tail with at least two external legs.

(ii) If $T$ is a two-legged tail we define the two-legged tail $T \circ K$ by

$$
\begin{aligned}
(T \circ K)(\psi ; \varphi ; \eta)=\int & \int: T\left(\psi ; \varphi^{\prime} ; \eta^{\prime}\right): \eta^{\prime}, C \\
& : \sum_{\substack{p_{1}, n_{1} \\
p_{3}+n_{3}=2}} K_{0\left(\begin{array}{c}
p_{1} 0 p_{3} \\
n_{1} 0 n_{3}
\end{array}\right)\left(\psi ; \varphi^{\prime}, \zeta^{\prime}, \varphi ; \eta^{\prime}, \xi^{\prime}, \eta\right)^{\cdot} \underset{\varphi^{\prime}, D}{\eta^{\prime}, C}} \\
& \times d \mu_{D}\left(\varphi^{\prime}\right) d \mu_{C}\left(\eta^{\prime}\right) .
\end{aligned}
$$

Remark IX.6. Again, a two-legged tail with two external legs is an end in the sense of Def. VI.5. If $K\left(\psi ; \zeta, \zeta^{\prime}, \varphi ; \xi, \xi^{\prime}, \eta\right)=U\left(\psi+\zeta+\zeta^{\prime}+\varphi ; \xi+\xi^{\prime}+\eta\right)-U\left(\psi+\zeta+\zeta^{\prime}+\right.$ $\left.\varphi ; \xi+\xi^{\prime}\right)$ for some Grassmann function $U(\psi ; \xi)$ then $T \circ K$ agrees with $T \circ \operatorname{Rung}(U)$ of Defs. VI.5 and VI.8.

Recall that we are interested in the two- and four-legged contributions to the Grassmann function $f^{\prime}(\psi)$ of Theorem VI.10. As in Def. VII.8 such contributions are extracted by

Definition IX.7. The operator $P$ maps $: f(\psi ; \zeta ; \xi): \xi, C$ to $f_{4,0,0}(\psi ; 0 ; 0)+f_{2,0,0}$ $(\psi ; 0 ; 0)$, when $f=\sum_{n_{0}, n_{1}, n_{2}} f_{n_{0}, n_{1}, n_{2}}$ with $f_{n_{0}, n_{1}, n_{2}} \in A\left[n_{0}, n_{1}, n_{2}\right]$.

Definition IX.8. Let $T(\psi ; \varphi ; \eta)$ be an n-legged tail. 
i) An element $v$ of the norm domain $\mathcal{N}_{d}$ is said to be an effective bound for $T$ if

$$
\begin{aligned}
& \quad N\left(\int: T(\psi ; \varphi ; \eta):_{\eta, C} h\left(\psi ; \varphi ; \eta ; \xi^{(1)}, \cdots, \xi^{(r)}\right) d \mu_{C}(\eta) ; \alpha\right) \leq \frac{v}{\alpha^{2}} N(h ; \alpha) \\
& N_{\operatorname{impr}}\left(\int: T(\psi ; \varphi ; \eta): \eta, C h\left(\psi ; \varphi ; \eta ; \xi^{(1)}, \cdots, \xi^{(r)}\right) d \mu_{C}(\eta) ; \alpha\right) \leq \frac{v}{\alpha^{2}} N_{\operatorname{impr}}(h ; \alpha) \\
& \text { for all Grassmann functions } h\left(\psi ; \varphi ; \eta ; \xi^{(1)}, \cdots, \xi^{(r)}\right) . \\
& \text { ii) For } v \in \mathcal{N}_{d}, \text { we write }
\end{aligned}
$$

$$
N_{\mathrm{eff}}(T ; \alpha) \leq v \quad \Longleftrightarrow \quad v \text { is an effective bound for } T .
$$

Remark IX.9. Proposition II.33 and the fact that $N_{\mathrm{impr}}(T ; \alpha) \leq N(T ; \alpha)$ imply

$$
N_{\text {eff }}(T ; \alpha) \leq N(T ; \alpha) .
$$

Lemma IX.10. Assume that $K\left(\psi ; \zeta, \zeta^{\prime}, \varphi ; \xi, \xi^{\prime}, \eta\right)$ has degree at most two in the variables $\zeta^{\prime}, \varphi, \xi^{\prime}, \eta$ and that $\alpha \geq 2$. Let $T$ be a two-legged tail with at least e external legs. If $e \geq 4$, then

$$
P Q(T) \mathcal{Q}_{K}(: f:)=P Q(T \circ K)(: f:),
$$

where $: f:$ is shorthand for $: f: \xi, C$. More generally, if $e \geq 2$, there exists a one-legged tail $t_{1}$ with at least $e+1$ external legs, a two-legged tail $t_{2}$ with at least $e+1$ external legs and a two-legged tail $\tau(\psi ; \varphi ; \eta)$ with at least $e+2$ external legs and degree two ${ }^{2}$ in $\eta$ such that for any Grassmann function $f(\psi ; \zeta ; \xi)$ the following holds:

Set

$$
\begin{aligned}
f^{\prime}(\psi)= & P\left[Q(T) \mathcal{Q}_{K}(: f:)-Q(T \circ K)(: f:)-Q\left(T \circ K, T_{1}(K)\right)(: f:)\right. \\
& \left.-Q\left(t_{1}+t_{2}+\tau\right)(: f:)\right]
\end{aligned}
$$

where

$$
T_{1}(K)=K_{2}\left(\begin{array}{c}
000 \\
002
\end{array}\right)+K_{2}\left(\begin{array}{c}
001 \\
001
\end{array}\right)
$$

Then

$$
N_{\text {impr }}\left(f^{\prime} ; \alpha\right) \leq \frac{2^{9} J}{\alpha^{8}} N(f ; 4 \alpha) N(T ; 2 \alpha) N(K ; 4 \alpha)\left(1+\frac{1}{\alpha^{2}} N(K ; 4 \alpha)\right) .
$$

Furthermore

$$
\begin{aligned}
N\left(t_{1}\right) & \leq \frac{1}{\alpha^{2}} N(T) N(K), \\
N\left(t_{2}\right) & \leq \frac{2}{\alpha^{2}} N(T) N(K), \\
N_{\mathrm{eff}}(\tau) & \leq \frac{4}{\alpha^{4}} N(T) N(K)^{2} .
\end{aligned}
$$

Proof. The proof is similar to that of Lemma VII.11. If : $f_{\ell}: \xi, C=Q(T) Q(K, \cdots, K)$ (: $f:$ ), with $\ell K$ 's, then, as $K$ is of degree at least two in $\psi, \zeta, \xi$ and $T$ is $2-$ legged with $e$ external legs, $f_{\ell}$ has degree at least $e+2 \ell-2$. Hence

$$
\begin{aligned}
& P\left[Q(T) \mathcal{Q}_{K}(: f:)\right]=P[Q(T) Q(K)(: f:)] \quad \text { if } e \geq 4, \\
& P\left[Q(T) \mathcal{Q}_{K}(: f:)\right]=P[Q(T) Q(K)(: f:)]+\frac{1}{2} P[Q(T) Q(K, K)(: f:)] \quad \text { if } e \geq 2 \text {. }
\end{aligned}
$$

\footnotetext{
${ }^{2}$ Hence $\tau$ is independent of $\varphi$.
} 
The contribution $P Q(T) Q\left(K_{\left.n_{0}\left(\begin{array}{l}p_{1} p_{2} p_{3} \\ n_{1} n_{2} n_{3}\end{array}\right)\right)}\right)$ vanishes unless

- $p_{1}+n_{1} \leq 2$, since otherwise $Q(T) Q\left(K \cdot\left(\begin{array}{c}p_{1} p_{2} p_{3} \\ n_{1} n_{2} n_{3}\end{array}\right)\right)$ is of degree at least one in $\zeta$ and $P$ sets $\zeta$ to zero.

- $n_{1} \in\{1,2\}$, since these fields must connect to $\eta$ fields of $T$ and $T$ has degree one or two in $\eta$.

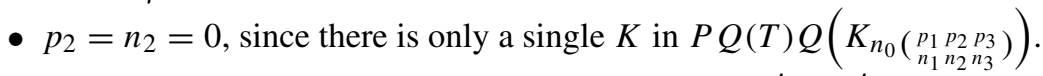

- $p_{3}+n_{3} \leq 2$ because $K$ is of degree at most two in $\zeta^{\prime}, \varphi, \xi^{\prime}, \eta$.

- $n_{3} \geq 1$ because $K$ is of degree at least one in $\eta$.

- $n_{0}+n_{1}+p_{1}+n_{3}+p_{3} \geq 4$ because $K$ is of degree at least four overall.

Hence

$$
\begin{aligned}
P Q(T) Q(K)= & P Q(T) Q\left(\sum_{\substack{p_{1}+n_{1}=2 \\
p_{3}+n_{3}=2}} K \cdot\left(\begin{array}{l}
p_{1} 0 p_{3} \\
n_{1} 0 n_{3}
\end{array}\right)\right)+P Q(T) Q\left(\sum_{p_{3}+n_{3}=2} K \cdot\left(\begin{array}{c}
00 p_{3} \\
10 n_{3}
\end{array}\right)\right) \\
& +P Q(T) Q\left(\sum_{p_{1}+n_{1}=2} K \cdot\left(\begin{array}{l}
p_{1} 00 \\
n_{1} 01
\end{array}\right)\right)+P Q(T) Q\left(K \cdot\left(\begin{array}{c}
000 \\
101
\end{array}\right)\right) \\
= & P\left[Q(T \circ K)+Q(k)+Q\left(t_{1}+t_{21}+t_{22}\right)\right],
\end{aligned}
$$

where

$$
\begin{aligned}
k(\psi ; \varphi ; \eta)= & \int T_{11}\left(\psi ; \varphi ; \eta^{\prime}\right)\left(\sum_{\substack{p_{3}+n_{3}=2 \\
n_{0} \geq 1}} K_{n_{0}}\left(\begin{array}{c}
00 p_{3} \\
10 n_{3}
\end{array}\right)\left(\psi ; 0,0, \varphi ; \eta^{\prime}, 0, \eta\right)\right) d \mu_{C}\left(\eta^{\prime}\right), \\
t_{1}(\psi ; \varphi ; \eta)= & \iint: T\left(\psi ; \varphi^{\prime} ; \eta^{\prime}\right): \eta^{\prime}\left(\sum_{\substack{p_{1}+n_{1}=2 \\
n_{0} \geq 1}} K_{n_{0}\left(\begin{array}{c}
p_{1} 00 \\
n_{1} 01
\end{array}\right)}\right) \\
& \left.\times\left(\psi ; \varphi^{\prime}, 0,0 ; \eta^{\prime}, 0, \eta\right)\right) d \mu_{D}\left(\varphi^{\prime}\right) d \mu_{C}\left(\eta^{\prime}\right) \\
& \quad \text { is a one-legged tail with at least } e+1 \text { external legs, } \\
t_{21}(\psi ; \varphi ; \eta)= & \iint: T\left(\psi ; \varphi^{\prime} ; \eta^{\prime}\right): \eta^{\prime}\left(\sum_{n_{0} \geq 1} \sum_{\substack{p_{1}+n_{1}=2 \\
p_{3}+n_{3}=2}} K_{n_{0}}\left(\begin{array}{c}
p_{1} 0 p_{3} \\
n_{1} 0 n_{3}
\end{array}\right)\right) \\
& \left.\times\left(\psi ; \varphi^{\prime}, 0, \varphi ; \eta^{\prime}, 0, \eta\right)\right) d \mu_{D}\left(\varphi^{\prime}\right) d \mu_{C}\left(\eta^{\prime}\right) \\
& \text { is a two-legged tail with at least } e+1 \text { external legs, } \\
t_{22}(\psi ; \varphi ; \eta)= & \int T_{11}\left(\psi ; \varphi ; \eta^{\prime}\right)\left(\sum_{n_{0} \geq 2} K_{n_{0}}\left(\begin{array}{c}
000 \\
101
\end{array}\right)\left(\psi ; 0,0,0 ; \eta^{\prime}, 0, \eta\right)\right) d \mu_{C}\left(\eta^{\prime}\right) \\
& \quad \text { is a two-legged tail with at least } e+2 \text { external legs. }
\end{aligned}
$$

If $e \geq 4$,

$$
P Q(k)=P Q\left(t_{1}\right)=P Q\left(t_{21}\right)=P Q\left(t_{22}\right)=0 .
$$

If $e \geq 2$, by Lemma II.31 and Prop. II.33,

$$
N\left(t_{1}\right), N\left(t_{21}\right), N\left(t_{22}\right) \leq \frac{1}{\alpha^{2}} N(T) N(K) .
$$

Furthermore $k$ has degree at least one in the variable $\eta$ and degree three in the variables $\varphi, \eta$. By Lemma IX.2 and Prop. II.33

$$
\begin{aligned}
N_{\text {impr }}(P Q(k)(: f:)) & \leq \frac{2^{5}}{\alpha^{6}} J N(k ; 2 \alpha) N(f ; 4 \alpha) \\
& \leq \frac{2^{3}}{\alpha^{8}} J N(T ; 2 \alpha) N(K ; 2 \alpha) N(f ; 4 \alpha) .
\end{aligned}
$$


We define the projection $P^{\prime}$ by

$$
P^{\prime}(f(\psi ; \zeta ; \xi))=f_{4}(\psi ; 0 ; 0)+f_{2}(\psi ; 0 ; 0) .
$$

Observe that

$$
\begin{aligned}
P[Q(T) Q(K, K)(: f:)] & =P^{\prime}[\tilde{Q}(T) Q(K, K)(: f:)(\psi ; \zeta, 0,0 ; \xi, 0,0)] \\
& =P^{\prime}[\tilde{Q}(T) Q(K, K)(: f:)(\psi ; 0,0,0 ; 0,0,0)]
\end{aligned}
$$

so that we can apply Prop. IX.3. Modulo a term whose improved norm $N_{\text {impr }}$ is bounded by $\frac{2^{9} J}{\alpha^{10}} N(f ; 4 \alpha) N(K ; 4 \alpha)^{2} N(T ; 2 \alpha)$

$$
\begin{aligned}
& P^{\prime}[\tilde{Q}(T) Q(K, K)(: f:)(\psi ; \zeta, 0,0 ; 0,0, \xi)] \\
& \quad=2 P\left[Q\left(\tilde{K}_{1}, K\right)(: f:)\right]+2 P\left[Q\left(\tilde{K}_{2}, K\right)(: f:)\right]+P[Q(\hat{K})(: f:)],
\end{aligned}
$$

where $\tilde{K}_{1}, \tilde{K}_{2}, \hat{K}$ are as in Prop. IX.3. Since $K_{\text {., } 1}$ has degree at least three in $\psi, \zeta, \xi$, the tail $\hat{K}$ has at least six external legs, so that $P[Q(\hat{K})(: f:)]=0$. Similarly, as $\tilde{K}_{2}$ has degree at least three in $\psi, \zeta, \xi$ and $K$ has degree at least two in $\psi, \zeta, \xi, P\left[Q\left(\tilde{K}_{2}, K\right)(: f:)\right]$ $=0$. As $\tilde{K}_{1}$ and $K$ have degree at most two in $\zeta^{\prime}, \varphi, \xi^{\prime}, \eta$ and degree at least four overall,

$$
\begin{aligned}
P\left[Q\left(\tilde{K}_{1}, K\right)(: f:)\right] & =P\left[Q\left(T_{1}\left(\tilde{K}_{1}\right), T_{1}(K)\right)(: f:)\right]+P[Q(\tau)(: f:)] \\
& =P\left[Q\left(T \circ K, T_{1}(K)\right)(: f:)\right]+P[Q(\tau)(: f:)],
\end{aligned}
$$

where

$$
\begin{aligned}
& \tau(\psi ; \varphi ; \eta)=\sum_{p_{2}+n_{2}=1} \iint\left(\tilde{K}_{1}\right)_{2\left(\begin{array}{c}
0 p_{2} 0 \\
0 n_{2} 1
\end{array}\right)}\left(\psi ; 0, \zeta^{\prime}, 0 ; 0, \xi^{\prime}, \eta\right) \\
& \times K_{2}\left(\begin{array}{c}
0 p_{2} 0 \\
0 n_{2} 1
\end{array}\right)\left(\psi ; 0, \zeta^{\prime}, 0 ; 0, \xi^{\prime}, \eta\right) d \mu_{D}\left(\zeta^{\prime}\right) d \mu_{C}\left(\xi^{\prime}\right)
\end{aligned}
$$

is a two-legged tail with at least $e+2$ external legs and degree two in $\eta$. By Prop. II.33,

$$
\begin{aligned}
& N\left(\sum_{p_{2}+n_{2}=1}\left(\tilde{K}_{1}\right)_{2}\left(\begin{array}{c}
0 p_{2} 0 \\
0 n_{2} 1
\end{array}\right)\left(\psi ; 0, \zeta^{\prime}, 0 ; 0, \xi^{\prime}, \eta\right)\right)
\end{aligned}
$$

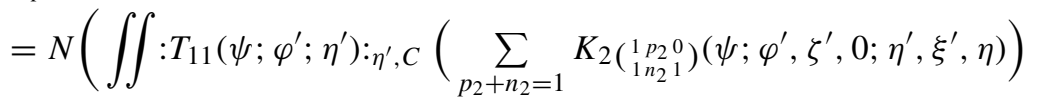

$$
\begin{aligned}
& \left.\times d \mu_{D}\left(\varphi^{\prime}\right) d \mu_{C}\left(\eta^{\prime}\right)\right) \\
& \leq \frac{1}{\alpha^{2}} N(T) N(K) \text {. }
\end{aligned}
$$

Therefore, by Prop. II.33, for any Grassmann function $h$,

$$
\begin{aligned}
& N\left(\iint: \tau(\psi ; \varphi ; \eta):_{\eta, C} h\left(\psi ; \varphi ; \eta ; \xi^{(1)}, \cdots, \xi^{(r)}\right) d \mu_{C}(\eta)\right) \\
& \quad \leq \frac{4}{\alpha^{4}} N\left(\sum_{p_{2}+n_{2}=1}\left(\tilde{K}_{1}\right)_{2}\left(\begin{array}{c}
0 p_{2} 0 \\
0 n_{2} 1
\end{array}\right)\left(\psi ; 0, \zeta^{\prime}, 0 ; 0, \xi^{\prime}, \eta\right)\right) N(K) N(h) \\
& \quad \leq \frac{4}{\alpha^{6}} N(T) N(K)^{2} N(h) .
\end{aligned}
$$


Similarly,

$$
\begin{aligned}
& \quad N_{\text {impr }}\left(\iint: \tau(\psi ; \varphi ; \eta):_{\eta, C} h\left(\psi ; \varphi ; \eta ; \xi^{(1)}, \cdots, \xi^{(r)}\right) d \mu_{C}(\eta)\right) \\
& \quad \leq \frac{4}{\alpha^{6}} N_{\text {impr }}(T) N_{\text {impr }}(K)^{2} N_{\text {impr }}(h) \\
& \quad \leq \frac{4}{\alpha^{6}} N(T) N(K)^{2} N_{\text {impr }}(h) .
\end{aligned}
$$

Therefore

$$
N_{\text {eff }}(\tau) \leq \frac{4}{\alpha^{4}} N(T) N(K)^{2} .
$$

Lemma IX.11. Assume that $K\left(\psi ; \zeta, \zeta^{\prime}, \varphi ; \xi, \xi^{\prime}, \eta\right)$ has degree at most two in the variables $\zeta^{\prime}, \varphi, \xi^{\prime}, \eta$. Let $T_{1}, T_{2}$ be two-legged tails. Then there exists a one-legged tail $t_{1}$, a two-legged tail t $t_{2}$ and a two-legged tail $\tau(\psi ; \varphi ; \eta)$ of degree two in $\eta$, each with at least four external legs, such that for any Grassmann function $f(\psi ; \zeta ; \xi)$ the following holds:

$$
f^{\prime}(\psi)=P\left[Q\left(T_{1}, T_{2}\right) \mathcal{Q}_{K}(: f:)-Q\left(T_{1} \circ K, T_{2} \circ K\right)(: f:)-Q\left(t_{1}+t_{2}+\tau\right)(: f:)\right] .
$$

Then, if $\alpha \geq 2$,

$$
N_{\mathrm{impr}}\left(f^{\prime}\right) \leq \frac{2^{9} J}{\alpha^{10}} N(f ; 4 \alpha) N\left(T_{1} ; 2 \alpha\right) N\left(T_{2} ; 2 \alpha\right) N(K ; 4 \alpha)\left(1+\frac{N(K ; 4 \alpha)}{\alpha^{2}}\right),
$$

and

$$
\begin{aligned}
N\left(t_{1}\right) & \leq \frac{4}{\alpha^{4}} N\left(T_{1}\right) N\left(T_{2}\right) N(K), \\
N\left(t_{2}\right) & \leq \frac{12}{\alpha^{4}} N\left(T_{1}\right) N\left(T_{2}\right) N(K), \\
N_{\text {eff }}(\tau) & \leq \frac{8}{\alpha^{6}} N\left(T_{1}\right) N\left(T_{2}\right) N(K)^{2} .
\end{aligned}
$$

Proof. The proof is similar to that of Lemma VII.12. Again, if

$$
: f_{\ell}: \xi, C=Q\left(T_{1}, T_{2}\right) Q(K, \cdots, K)(: f:)
$$

with $\ell K$ 's, then, as $K$ is of degree at least two in $\psi, \zeta, \xi$ and $T_{1}, T_{2}$ are 2-legged with at least two external legs, $f_{\ell}$ has degree at least $4+2 \ell-4$. Hence

$$
P\left[Q\left(T_{1}, T_{2}\right) \mathcal{Q}_{K}(: f:)\right]=P\left[Q\left(T_{1}, T_{2}\right) Q(K)(: f:)\right]+\frac{1}{2} P\left[Q\left(T_{1}, T_{2}\right) Q(K, K)(: f:)\right] .
$$

The contribution $P Q\left(T_{1}, T_{2}\right) Q\left(K_{n_{0}}\left(\begin{array}{c}p_{1} p_{2} p_{3} \\ n_{1} n_{2} n_{3}\end{array}\right)\right)$ vanishes unless

- $n_{0}=0$ because $T_{1}$ and $T_{2}$ are each of degree at least two in $\psi$.

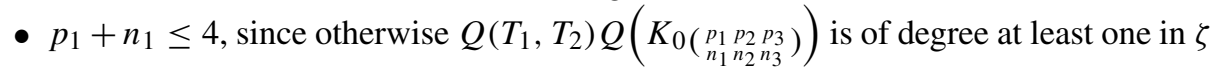
and $P$ sets $\zeta$ to zero.

- $n_{1} \geq 2$ since these fields must connect to $\eta$ fields of $T_{1}, T_{2}$ which have combined degree at least two in $\eta$.

- $p_{2}=n_{2}=0$ since there is only a single $K$ in $P Q\left(T_{1}, T_{2}\right) Q\left(K_{n_{0}}\left(\begin{array}{c}p_{1} p_{2} p_{3} \\ n_{1} n_{2} n_{3}\end{array}\right)\right)$.

- $p_{3}+n_{3} \leq 2$ because $K$ is of degree at most two in $\zeta^{\prime}, \varphi, \xi^{\prime}, \eta$.

- $n_{3} \geq 1$ because $K$ is of degree at least one in $\eta$.

- $n_{1}+p_{1}+n_{3}+p_{3} \geq 4$ because $K$ is of degree at least four overall. 
Hence

$$
P\left[Q\left(T_{1}, T_{2}\right) Q(K)(: f:)\right]=P\left[Q\left(t_{11}+t_{21}+t_{22}+t_{23}\right)(: f:)\right]+P[Q(k)(: f:)],
$$

where

$$
\begin{aligned}
& t_{11}(\psi ; \varphi ; \eta)=\iint: T_{1}\left(\psi ; \varphi^{\prime} ; \eta^{\prime}\right) T_{2}\left(\psi ; \varphi^{\prime} ; \eta^{\prime}\right): \underset{\varphi^{\prime}, D}{\eta^{\prime}, C} \\
& \times\left(\sum_{p_{1}+n_{1}=4} K_{0\left(\begin{array}{c}
p_{1} 00 \\
n_{1} 01
\end{array}\right)}\left(0 ; \varphi^{\prime}, 0,0 ; \eta^{\prime}, 0, \eta\right)\right) d \mu_{D}\left(\varphi^{\prime}\right) d \mu_{C}\left(\eta^{\prime}\right), \\
& t_{21}(\psi ; \varphi ; \eta)=\iint: T_{1}\left(\psi ; \varphi^{\prime} ; \eta^{\prime}\right) T_{2}\left(\psi ; \varphi^{\prime} ; \eta^{\prime}\right) \underset{\substack{\varphi^{\prime}, D \\
\eta^{\prime}, C}}{.} \\
& \times\left(\sum_{\substack{p_{1}+n_{1}=4 \\
p_{3}+n_{3}=2}} K_{0\left(\begin{array}{c}
p_{1} 0 p_{3} \\
n_{1} 0 n_{3}
\end{array}\right)}\left(0 ; \varphi^{\prime}, 0, \varphi ; \eta^{\prime}, 0, \eta\right)\right) d \mu_{D}\left(\varphi^{\prime}\right) d \mu_{C}\left(\eta^{\prime}\right), \\
& t_{22}(\psi ; \varphi ; \eta)=\iint: T_{1}\left(\psi ; \varphi ; \eta^{\prime}\right) T_{2}\left(\psi ; \varphi^{\prime} ; \eta^{\prime}\right):_{\eta^{\prime}, C}
\end{aligned}
$$

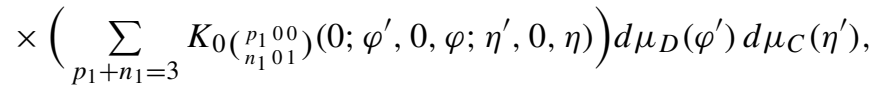

$$
\begin{aligned}
& t_{23}(\psi ; \varphi ; \eta)=\iint: T_{1}\left(\psi ; \varphi^{\prime} ; \eta^{\prime}\right) T_{2}\left(\psi ; \varphi ; \eta^{\prime}\right){ }_{\eta^{\prime}, C} \\
& \times\left(\sum_{p_{1}+n_{1}=3} K_{0\left(\begin{array}{c}
p_{1} 00 \\
n_{1} 01
\end{array}\right)}\left(0 ; \varphi^{\prime}, 0, \varphi ; \eta^{\prime}, 0, \eta\right)\right) d \mu_{D}\left(\varphi^{\prime}\right) d \mu_{C}\left(\eta^{\prime}\right), \\
& k(\psi ; \varphi ; \eta)=\iint: T_{1}\left(\psi ; \varphi ; \eta^{\prime}\right) T_{2}\left(\psi ; \varphi ; \eta^{\prime}\right){ }_{\eta_{\eta^{\prime}, C}} \\
& \times\left(\sum_{\substack{p_{3}+n_{3}=2 \\
n_{3} \geq 1}} K_{0\left(\begin{array}{c}
00 p_{3} \\
20 n_{3}
\end{array}\right)}\left(0 ; 0,0, \varphi ; \eta^{\prime}, 0, \eta\right)\right) d \mu_{C}\left(\eta^{\prime}\right) .
\end{aligned}
$$

Here, each $t_{i j}$ is a $i$-legged tail with at least four external legs, that, by Lemma II.31 and Prop. II.33, with $\ell=2$, fulfills

$$
N\left(t_{i j}\right) \leq \frac{4}{\alpha^{4}} N\left(T_{1}\right) N\left(T_{2}\right) N(K)
$$

Furthermore $k$ has degree at least one in the variable $\eta$ and degree three in the variables $\varphi, \eta$. By Lemma IX.2 and Prop. II.33 with $\ell=2$ and $\alpha$ replaced by $2 \alpha$,

$$
\begin{aligned}
N_{\text {impr }}(P Q(k)(: f:)) & \leq \frac{2^{5}}{\alpha^{6}} J N(k ; 2 \alpha) N(f ; 4 \alpha) \\
& \leq \frac{2^{3}}{\alpha^{10}} J N\left(T_{1} ; 2 \alpha\right) N\left(T_{2} ; 2 \alpha\right) N(K ; 2 \alpha) N(f ; 4 \alpha) .
\end{aligned}
$$

By Prop. VIII.13.

$$
\begin{aligned}
& Q\left(T_{1}, T_{2}\right) Q(K, K)(: f:) \\
& =\cdot \iint: T_{1}(\psi ; \varphi ; \eta):_{\eta, C}: g(\psi ; \zeta, 0, \varphi ; \xi, 0, \eta):_{\varphi, D} d \mu_{D}(\varphi) d \mu_{C}(\eta)_{\cdot \xi, C}^{\cdot}
\end{aligned}
$$

where $g=\tilde{Q}\left(T_{2}\right) Q(K, K)(: f:)$. In particular

$$
\begin{array}{rl}
P & Q\left(T_{1}, T_{2}\right) Q(K, K)(: f:) \\
& =P^{\prime} \iint: T_{1}(\psi ; \varphi ; \eta):_{\eta, C}: g(\psi ; 0,0, \varphi ; 0,0, \eta): \underset{\eta, C}{\eta_{, C}} d \mu_{D}(\varphi) d \mu_{C}(\eta) .
\end{array}
$$




\section{By Prop. IX.3,}

$g(\psi ; 0,0, \varphi ; 0,0, \eta)=\tilde{g}_{1}(\psi ; \varphi ; \eta)+\tilde{g}_{2}(\psi ; \varphi ; \eta)+\hat{g}(\psi ; \varphi ; \eta)+h(\psi ; \varphi ; \eta)$,

where

$$
\begin{aligned}
\tilde{g}_{1}(\psi ; \varphi ; \eta) & =2 Q\left(\tilde{K}_{1}, K\right)(: f:)(\psi ; \varphi ; \eta), \\
\tilde{g}_{2}(\psi ; \varphi ; \eta) & =2 Q\left(\tilde{K}_{2}, K\right)(: f:)(\psi ; \varphi ; \eta), \\
\hat{g}(\psi ; \varphi ; \eta) & =Q(\hat{K})(: f:)(\psi ; \varphi ; \eta),
\end{aligned}
$$

and

$$
N_{\text {impr }}(h) \leq \frac{2^{9} J}{\alpha^{10}} N(f ; 4 \alpha) N(K ; 4 \alpha)^{2} N\left(T_{2} ; 2 \alpha\right) .
$$

By Lemma II.31 and Prop. II.33, the improved norm of

$$
\iint: T_{1}(\psi ; \varphi ; \eta): \eta, C: h(\psi ; \varphi ; \eta): \underset{\eta, C}{\eta, D} d \mu_{D}(\varphi) d \mu_{C}(\eta)
$$

is bounded by

$$
\frac{1}{\alpha^{2}} N_{\text {impr }}\left(T_{1}\right) N_{\text {impr }}(h) \leq \frac{2^{9} J}{\alpha^{12}} N(f ; 4 \alpha) N(K ; 4 \alpha)^{2} N\left(T_{1} ; \alpha\right) N\left(T_{2} ; 2 \alpha\right) .
$$

Observe that $\hat{g}$ has degree at least six, so that

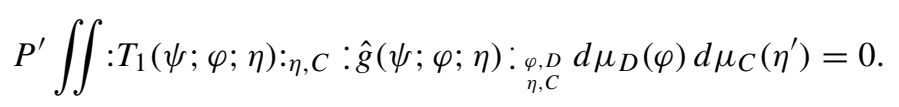

Similarly, $\tilde{g}_{2}$ has degree at least five, so that

$$
P^{\prime} \iint: T_{1}(\psi ; \varphi ; \eta): \eta, C: \tilde{g}_{2}(\psi ; \varphi ; \eta) \underset{\eta, \mathcal{}, D}{: D} d \mu_{D}(\varphi) d \mu_{C}\left(\eta^{\prime}\right)=0 .
$$

Finally, the contribution to $\tilde{g}_{1}$ with $\psi$-degree two and overall degree four is $\tilde{g}_{11}+\tilde{g}_{12}$, where

$$
\begin{aligned}
& \tilde{g}_{11}=2 Q\left(T_{2} \circ K, \sum_{\substack{p_{1}+n_{1}=2 \\
p_{3}+n_{3}=2}} K_{\left.0\left(\begin{array}{c}
p_{1} 0 p_{3} \\
n_{1} 0 n_{3}
\end{array}\right)\right)(: f:),} \tilde{g}_{12}=2 Q\left(p, \sum_{\substack{p_{1}+n_{1}=2 \\
p_{2}+n_{2}=1}} K_{0}\left(\begin{array}{c}
p_{1} p_{2} 0 \\
n_{1} n_{2}
\end{array}\right)\right)(: f:),\right.
\end{aligned}
$$

where

$$
\begin{aligned}
p\left(\psi ; \zeta^{\prime}, \varphi ; \xi^{\prime}, \eta\right)= & \iint: T_{2}\left(\psi ; \varphi^{\prime} ; \eta^{\prime}\right):_{\eta^{\prime}, C} \\
& \times\left(\sum_{\substack{p_{1}+n_{1}=2 \\
p_{2}+n_{2}=1}} K_{0}\left(\begin{array}{c}
p_{1} p_{2} 0 \\
n_{1} n_{2} 1
\end{array}\right)\left(\psi ; \varphi^{\prime}, \zeta^{\prime}, \varphi ; \eta^{\prime}, \xi^{\prime}, \eta\right)\right) d \mu_{D}\left(\varphi^{\prime}\right) d \mu_{C}\left(\eta^{\prime}\right) .
\end{aligned}
$$

Then

$$
\begin{aligned}
& P^{\prime} \iint: T_{1}(\psi ; \varphi ; \eta):_{\eta, C}: \tilde{g}_{11}(\psi ; \varphi ; \eta): \underset{\varphi, D}{\eta, C} d \mu_{D}(\varphi) d \mu_{C}(\eta) \\
& \quad=2 P Q\left(T_{1} \circ K, T_{2} \circ K\right)(: f:)
\end{aligned}
$$


while

$$
P^{\prime} \iint: T_{1}(\psi ; \varphi ; \eta): \eta, C: \tilde{g}_{12}(\psi ; \varphi ; \eta): \underset{\eta, C}{ } d \mu_{D}(\varphi) d \mu_{C}(\eta)=P Q(\tau)(: f:)
$$

with the two-legged tail, with four external legs and degree two in $\eta$,

$$
\tau=2 \int p\left(\psi ; \zeta^{\prime}, \varphi ; \xi^{\prime}, \eta\right) p^{\prime}\left(\psi ; \zeta^{\prime}, \varphi ; \xi^{\prime}, \eta\right) d \mu_{D}\left(\zeta^{\prime}\right) d \mu_{C}\left(\xi^{\prime}\right),
$$

where $p$ was defined above and

$$
\begin{aligned}
p^{\prime}\left(\psi ; \zeta^{\prime}, \varphi ; \xi^{\prime}, \eta\right)= & \iint: T_{1}\left(\psi ; \varphi^{\prime \prime}, \eta^{\prime \prime}\right): \eta^{\prime \prime}, C \\
& \times\left(\sum_{\substack{p_{1}+n_{1}=2 \\
p_{2}+n_{2}=1}} K_{0}\left(\begin{array}{c}
p_{1} p_{2} 0 \\
n_{1} n_{2} 1
\end{array}\right)\left(\psi ; \varphi^{\prime \prime}, \zeta^{\prime}, \varphi ; \eta^{\prime \prime}, \xi^{\prime}, \eta\right)\right) d \mu_{D}\left(\varphi^{\prime \prime}\right) d \mu_{C}\left(\eta^{\prime \prime}\right) .
\end{aligned}
$$

By Lemma II.31 and Prop. II.33,

$$
\begin{aligned}
N(p) & \leq \frac{1}{\alpha^{2}} N\left(T_{2}\right) N(K), \\
N\left(p^{\prime}\right) & \leq \frac{1}{\alpha^{2}} N\left(T_{1}\right) N(K) .
\end{aligned}
$$

By Lemma II.31 and Prop. II.33, with $\ell=2$,

$$
\begin{aligned}
& N\left(\iint: \tau(\psi ; \varphi ; \eta):_{\eta, C} h\left(\psi ; \varphi ; \eta ; \xi^{(1)}, \cdots, \xi^{(r)}\right) d \mu_{C}(\eta)\right) \\
& \quad \leq \frac{8}{\alpha^{4}} N(p) N\left(p^{\prime}\right) N(h) \\
& \quad \leq \frac{8}{\alpha^{8}} N\left(T_{1}\right) N\left(T_{2}\right) N(K)^{2} N(h)
\end{aligned}
$$

for all Grassmann functions $h$. A similar estimate applies for the $N_{\text {impr }}$ norm so that

$$
N_{\text {eff }}(\tau) \leq \frac{8}{\alpha^{6}} N\left(T_{1}\right) N\left(T_{2}\right) N(K)^{2} .
$$

Lemma IX.12. Assume that $K$ has degree at most two in the variables $\zeta^{\prime}, \varphi, \xi^{\prime}, \eta$. Let $T$ be a one-legged tail with at least three external legs. Then there is a two-legged tail $t_{2}$ with at least four external legs such that for all Grassmann functions $f(\psi ; \zeta, \xi)$,

$$
P\left[Q(T) \mathcal{Q}_{K}(: f:)\right]=P\left[Q\left(t_{2}\right)(: f:)\right]
$$

and

$$
N\left(t_{2}\right) \leq \frac{2}{\alpha^{2}} N(T) N(K) .
$$

Proof. The proof is similar to that of Lemma VII.13. Since $T$ is one-legged and $K$ has degree at least four

$$
\begin{aligned}
P Q(T) \mathcal{Q}_{K} & =P Q(T) Q(K) \\
& =P Q(T) Q\left(\sum_{p_{3}+n_{3}=2} K_{\left.1\left(\begin{array}{c}
00 \\
10 n_{3}
\end{array}\right)\right)=P Q\left(t_{2}\right)}\right.
\end{aligned}
$$

with

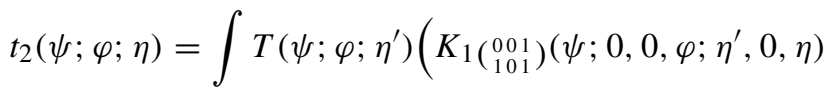

$$
\begin{aligned}
& \left.+K_{1\left(\begin{array}{c}
000 \\
102
\end{array}\right)}\left(\psi ; 0,0,0 ; \eta^{\prime}, 0, \eta\right)\right) d \mu_{C}\left(\eta^{\prime}\right)
\end{aligned}
$$


Definition IX.13. The two-legged tails $T_{\ell}(K)$ are recursively defined as follows: $T_{1}(K)$ was defined in Lemma IX.10, and

$$
T_{\ell+1}(K)=T_{\ell} \circ K \quad \text { for } \ell \geq 1
$$

Remark IX.14. (i) Clearly $T_{\ell}(K)$ has two external legs. Using Lemma II.31 and Prop. II.33 one proves by induction that for $\alpha \geq 2$,

$$
N\left(T_{\ell}(K)\right) \leq \frac{1}{\alpha^{2 \ell-2}} N(K)^{\ell} .
$$

(ii) If $K\left(\psi ; \zeta, \zeta^{\prime}, \varphi ; \xi, \xi^{\prime}, \eta\right)=U\left(\psi+\zeta+\zeta^{\prime}+\varphi ; \xi+\xi^{\prime}+\eta\right)-U\left(\psi+\zeta+\zeta^{\prime}+\varphi ; \xi+\xi^{\prime}\right)$ for some even Grassmann function $U(\psi ; \xi)$ then, by Remark IX.6, $T_{\ell}(K)=T_{\ell}(U)$. Recall that $T_{\ell}(U)$ was defined in Definition VI.8.

IX.3. Proof of Theorem VI.10 in the General Case. First, we prove the analog of Prop. VII.16 for the enlarged algebra.

Proposition IX.15. Let $K\left(\psi ; \zeta ; \xi, \xi^{\prime}, \eta\right)$ be an even Grassmann function that vanishes for $\eta=0$ and has degree at least four overall. Set

$$
\bar{K}\left(\psi ; \zeta, \zeta^{\prime}, \varphi ; \xi, \xi^{\prime}, \eta\right)=K\left(\psi ; \zeta+\zeta^{\prime}+\varphi ; \xi, \xi^{\prime}, \eta\right)
$$

Assume that $\alpha \geq 8$ and $N(\bar{K} ; 4 \alpha)_{0}<\frac{2 \alpha}{3}$. Furthermore let $f(\psi ; \zeta ; \xi)$ be a Grassmann function. For each $n \geq 1$ there exists a Grassmann function $h_{n}(\psi ; f)$, a one-legged tail $t_{1}$, a two-legged tail $t_{2}$, each with at least three external legs and a two-legged tail $\tau(\psi ; \varphi ; \eta)$ with at least four external legs and of degree two in $\eta$ such that

$$
\begin{aligned}
& P \int \operatorname{Ev} \mathcal{R}_{: K:_{\zeta, D}, C}^{n}(: f: \underset{\zeta, D}{:, C}) d \mu_{D}(\zeta)=P\left[Q\left(T_{n}(\bar{K})\right)(: f: \xi, C)\right. \\
& +\frac{1}{2} \sum_{\substack{\ell, \ell^{\prime} \prime 1 \\
\max \left\{\ell, \ell^{\prime}\right\}=n}} Q\left(T_{\ell}(\bar{K}), T_{\ell^{\prime}}(\bar{K})\right)(: f: \xi, C) \\
& \left.+Q\left(t_{1}+t_{2}+\tau\right)(: f: \xi, C)\right]+h_{n}(\psi ; f)
\end{aligned}
$$

and

$$
\begin{aligned}
N\left(t_{1}\right), N\left(t_{2}\right) & \leq 4\left(\frac{8}{\alpha^{2}}\right)^{n-1} \frac{N(\bar{K} ; \alpha)^{n}}{1-\frac{2}{\alpha^{2}} N(\bar{K} ; \alpha)}, \quad N_{\text {eff }}(\tau) \leq \frac{8}{\alpha^{2 n}} \frac{N(\bar{K} ; \alpha)^{n+1}}{1-\frac{1}{\alpha^{2}} N(\bar{K} ; \alpha)}, \\
N_{\text {impr }}\left(h_{n}(\psi ; f) ; \alpha\right) & \leq \frac{2^{10} J}{\alpha^{5}} \sum_{m=0}^{n-1} \frac{1}{\alpha^{n-m}} \frac{N(\bar{K} ; 4 \alpha)^{n-m}}{1-\frac{3}{2 \alpha} N(\bar{K} ; 4 \alpha)} N\left(F_{m}(\psi ; f) ; 4 \alpha\right),
\end{aligned}
$$

where

$$
: F_{m}(\psi ; f): \underset{\zeta, D}{\xi, C}=\operatorname{Ev} \mathcal{R}_{: K: \zeta, D, C}^{m}(: f: \underset{\zeta, D}{\xi, C}) .
$$

Proof. Decompose

$$
\bar{K}=K^{\prime}+K^{\prime \prime},
$$

where $K^{\prime}$ has degree at most two in the variables $\zeta^{\prime}, \xi^{\prime}, \varphi, \eta$ and $K^{\prime \prime}$ has degree at least three in these variables.

We perform induction on $n$. For $n=1$, by Lemma VIII.12.ii,

$$
\operatorname{Ev} \mathcal{R}_{: K:_{\zeta, D}, C}\left(: f::_{\zeta, D}^{\xi, C}\right)=: \mathcal{Q}_{\bar{K}}(: f: \xi, C):_{\zeta, D},
$$


and therefore

$$
\int \operatorname{Ev} \mathcal{R}_{: K: \zeta, D}, C(: f: \xi, C) d \mu_{D}(\zeta)=\mathcal{Q}_{\bar{K}}(: f:, \xi, C)(\psi, 0 ; \xi) .
$$

Set

$$
h_{1}(\psi ; f)=P\left(\mathcal{Q}_{\bar{K}}-\mathcal{Q}_{K^{\prime}}\right)(: f: \xi, C)
$$

By Prop. IX.1,

$$
N_{\text {impr }}\left(h_{1}(\psi ; f)\right) \leq \frac{2^{5} J}{\alpha^{6}} N(f ; 4 \alpha) \frac{N(\bar{K} ; 2 \alpha)}{1-\frac{1}{\alpha} N(\bar{K} ; 2 \alpha)} .
$$

Since $K^{\prime}$ has degree at most two in the variables $\zeta^{\prime}, \varphi, \xi^{\prime}, \eta$ and degree at least four overall

$$
\begin{aligned}
P \mathcal{Q}_{K^{\prime}}= & P Q\left(K \cdot\left(\begin{array}{c}
000 \\
001
\end{array}\right)+K \cdot\left(\begin{array}{c}
000 \\
002
\end{array}\right)+K \cdot\left(\begin{array}{c}
001 \\
001
\end{array}\right)\right) \\
& +\frac{1}{2} P Q\left(K_{2}\left(\begin{array}{c}
000 \\
011
\end{array}\right)+K_{2}\left(\begin{array}{c}
010 \\
001
\end{array}\right), K_{2}\left(\begin{array}{c}
000 \\
011
\end{array}\right)+K_{2}\left(\begin{array}{c}
010 \\
001
\end{array}\right)\right) \\
& +\frac{1}{2} P Q\left(K_{2}\left(\begin{array}{c}
000 \\
002
\end{array}\right)+K_{2}\left(\begin{array}{c}
001 \\
001
\end{array}\right), K_{2}\left(\begin{array}{c}
000 \\
002
\end{array}\right)+K_{2}\left(\begin{array}{c}
001 \\
001
\end{array}\right)\right) \\
= & P Q\left(T_{1}(\bar{K})\right)+P Q\left(t_{1}+t_{2}\right)+\frac{1}{2} P Q\left(T_{1}(\bar{K}), T_{1}(\bar{K})\right)
\end{aligned}
$$

with

$$
\begin{aligned}
t_{1} & =K_{3}\left(\begin{array}{c}
000 \\
001
\end{array}\right)+K_{4}\left(\begin{array}{c}
000 \\
001
\end{array}\right), \\
t_{2} & =K_{3}\left(\begin{array}{c}
000 \\
002
\end{array}\right)+K_{4}\left(\begin{array}{c}
000 \\
002
\end{array}\right)+K_{3}\left(\begin{array}{c}
001 \\
001
\end{array}\right)+K_{4}\left(\begin{array}{c}
001 \\
001
\end{array}\right)+\frac{1}{2} \int K_{2}\left(\begin{array}{c}
000 \\
011
\end{array}\right) K_{2}\left(\begin{array}{c}
000 \\
011
\end{array}\right) d \mu_{C}\left(\xi^{\prime}\right), \\
\tau & =\frac{1}{2} \int K_{\left.2\left(\begin{array}{c}
010 \\
001
\end{array}\right) K_{2(} \begin{array}{c}
010 \\
001
\end{array}\right) d \mu_{D}\left(\zeta^{\prime}\right) .}
\end{aligned}
$$

In particular

$$
\begin{aligned}
N\left(t_{1}\right) & \leq N(\bar{K}), \\
N\left(t_{2}\right) & \leq N(\bar{K})+\frac{1}{2 \alpha^{2}} N(\bar{K})^{2} \leq \frac{N(\bar{K})}{1-\frac{1}{2 \alpha^{2}} N(\bar{K})}, \\
N_{\text {eff }}(\tau) & \leq \frac{1}{2} \frac{4}{\alpha^{2}} N(\bar{K})^{2} \leq \frac{2}{\alpha^{2}} N(\bar{K})^{2} .
\end{aligned}
$$

Proposition, II.33, with $\ell=1$, was used to bound the last term of $t_{2}$. Lemma II.31 and Prop. II.33, with $\ell=2$, were used to bound $\int: \tau(\psi ; \varphi ; \eta):_{\eta, C} h\left(\psi ; \varphi ; \eta ; \xi^{(1)}, \cdots, \xi^{(r)}\right)$ $d \mu_{C}(\eta)$ as in Definition IX.8.

Now assume that the statement of the lemma is true for $n$. By Remark VIII.6.ii,

$$
\operatorname{Ev} \mathcal{R}_{: K: \zeta, D, C}^{n+1}(: f: \underset{\zeta, D}{:, C})=\operatorname{Ev} \mathcal{R}_{: K: \zeta, D}^{n}\left(: F_{1}(\psi ; f): \underset{\xi, C}{\xi, C}\right) .
$$

By Lemma VIII.12.ii,

$$
: F_{1}(\psi ; f): \xi, C=Q_{\bar{K}}(: f: \xi, C) .
$$

The induction hypothesis therefore implies that

$$
\begin{aligned}
& P \int \operatorname{Ev} \mathcal{R}_{: K: \zeta, D}^{n+1}\left(: f:_{\zeta, D}\right) d \mu_{D}(\zeta) \\
& \quad=P \int \operatorname{Ev} \mathcal{R}_{: K:_{\zeta, D}, C}^{n}\left(: \mathcal{Q}_{\bar{K}}(: f: \xi, C):_{\zeta, D}\right) d \mu_{D}(\zeta)
\end{aligned}
$$




$$
\begin{aligned}
= & h_{n}\left(\psi ; F_{1}(\psi ; f)\right)+P Q\left(T_{n}(\bar{K})\right)\left(\mathcal{Q}_{\bar{K}}(: f: \xi, C)\right) \\
& +\frac{1}{2} \sum_{\substack{\ell, \ell^{\prime} \geq 1 \\
\max \left\{\ell^{\prime} \ell^{\prime}\right\}=n}} P Q\left(T_{\ell}(\bar{K}), T_{\ell^{\prime}}(\bar{K})\right)\left(\mathcal{Q}_{\bar{K}}(: f: \xi, C)\right) \\
& +P Q\left(t_{1}+t_{2}+\tau\right)\left(\mathcal{Q}_{\bar{K}}(: f: \xi, C)\right)
\end{aligned}
$$

with

$$
N\left(t_{1}\right), N\left(t_{2}\right) \leq 4\left(\frac{8}{\alpha^{2}}\right)^{n-1} \frac{N(\bar{K})^{n}}{1-\frac{2}{\alpha^{2}} N(\bar{K})}, \quad N_{\text {eff }}(\tau) \leq \frac{8}{\alpha^{2 n}} \frac{N(\bar{K})^{n+1}}{1-\frac{1}{\alpha^{2}} N(\bar{K})}
$$

and

$$
N_{\text {impr }}\left(h_{n}\left(\psi ; F_{1}(\psi ; f)\right)\right) \leq \frac{2^{10} J}{\alpha^{5}} \sum_{m=0}^{n-1} \frac{1}{\alpha^{n-m}} \frac{N(\bar{K} ; 4 \alpha)^{n-m}}{1-\frac{3}{2 \alpha} N(\bar{K} ; 4 \alpha)} N\left(F_{m}\left(\psi ; F_{1}(f ; \psi)\right) ; 4 \alpha\right) .
$$

By Remark VIII.6.ii,

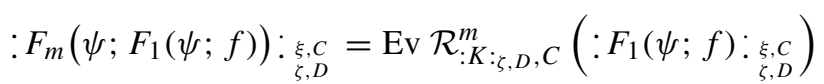

$$
\begin{aligned}
& =\operatorname{Ev} \mathcal{R}_{: K: \zeta, D}^{m}, C\left(\operatorname{Ev} \mathcal{R}_{: K:_{\zeta, D}, C}(: f: \underset{\zeta, D}{: \xi, C})\right) \\
& =\operatorname{Ev} \mathcal{R}_{: K:_{\zeta, D}, C}^{m+1}(: f: \underset{\zeta, D}{\xi, C}) \\
& =: F_{m+1}(\psi ; f): \underset{\xi, D}{\xi, C} \text {. }
\end{aligned}
$$

Therefore

$$
N_{\text {impr }}\left(h_{n}\left(\psi ; F_{1}(\psi ; f)\right)\right) \leq \frac{2^{10} J}{\alpha^{5}} \sum_{m=1}^{n} \frac{1}{\alpha^{n+1-m}} \frac{N(\bar{K} ; 4 \alpha)^{n+1-m}}{1-\frac{3}{2 \alpha} N(\bar{K} ; 4 \alpha)} N\left(F_{m}(\psi ; f) ; 4 \alpha\right) .
$$

Let $g_{0}$ be the difference between

$$
\begin{aligned}
& P\left[Q\left(T_{n}(\bar{K})\right)\left(\mathcal{Q}_{\bar{K}}(: f: \xi, C)\right)+\frac{1}{2} \sum_{\substack{\ell, \ell^{\prime} \geq 1 \\
\max \left\{, \ell \bar{\ell}^{\prime}\right\}=n}} Q\left(T_{\ell}(\bar{K}), T_{\ell^{\prime}}(\bar{K})\right)\left(\mathcal{Q}_{\bar{K}}(: f: \xi, C)\right)\right. \\
& \left.\quad+Q\left(t_{1}+t_{2}+\tau\right)\left(\mathcal{Q}_{\bar{K}}(: f: \xi, C)\right)\right]
\end{aligned}
$$

and

$$
\begin{aligned}
P & {\left[Q\left(T_{n}(\bar{K})\right)\left(\mathcal{Q}_{K^{\prime}}(: f: \xi, C)\right)+\frac{1}{\substack{\ell, \ell^{\prime} \geq 1 \\
\max \left\{\ell, \ell^{\prime}\right\}=n}} \underset{ }{ } Q\left(T_{\ell}(\bar{K}), T_{\ell^{\prime}}(\bar{K})\right)\left(\mathcal{Q}_{K^{\prime}}(: f: \xi, C)\right)\right.} \\
& \left.+Q\left(t_{1}+t_{2}+\tau\right)\left(\mathcal{Q}_{K^{\prime}}(: f: \xi, C)\right)\right] .
\end{aligned}
$$

Let $: f^{\prime \prime}: \xi, D=\mathcal{Q}_{\bar{K}}(: f: \xi, C)-\mathcal{Q}_{K^{\prime}}(: f: \xi, C)$. By Lemma VIII.14 and Def. IX.8

$$
\begin{aligned}
N_{\text {impr }}\left(g_{0}\right) \leq & \frac{1}{\alpha} N_{\text {impr }}\left(f^{\prime \prime} ; \alpha\right)\left[N\left(T_{n}(\bar{K})\right)+\frac{1}{\alpha} \sum_{\substack{\left.\ell, \ell^{\prime} \geq 1 \\
\max \ell, \ell^{\prime}\right\}=n}} N\left(T_{\ell}(\bar{K})\right) N\left(T_{\ell^{\prime}}(\bar{K})\right)\right. \\
& \left.+N\left(t_{1}\right)+N\left(t_{2}\right)+v\right]
\end{aligned}
$$


if $v$ is any effective bound for $\tau$. By Prop. IX.1, Remark IX.14 and the induction hypothesis,

$$
\begin{aligned}
N_{\text {impr }}\left(g_{0}\right) \leq & \frac{2^{5} J}{\alpha^{7}} N(f ; 4 \alpha) \frac{N(\bar{K} ; 2 \alpha)}{1-\frac{1}{\alpha} N(\bar{K} ; 2 \alpha)}\left[\frac{1}{\alpha^{2 n-2}} N(\bar{K})^{n}\left(1+2 \sum_{\ell=1}^{n} \frac{N(\bar{K})^{\ell}}{\alpha^{2 \ell-1}}\right)\right. \\
& \left.+8\left(\frac{8}{\alpha^{2}}\right)^{n-1} \frac{N(\bar{K})^{n}}{1-\frac{2}{\alpha^{2}} N(\bar{K})}+\frac{8}{\alpha^{2 n}} \frac{N(\bar{K})^{n+1}}{1-\frac{1}{\alpha^{2}} N(\bar{K})}\right] .
\end{aligned}
$$

Since

$$
\begin{aligned}
1+2 \sum_{\ell=1}^{n} \frac{N(\bar{K})^{\ell}}{\alpha^{2 \ell-1}} & =1+2 \alpha \sum_{\ell=1}^{n} \frac{N(\bar{K})^{\ell}}{\alpha^{2 \ell}} \leq 1+2 \alpha \frac{\frac{1}{\alpha^{2}} N(\bar{K})}{1-\frac{1}{\alpha^{2}} N(\bar{K})}=\frac{1-\frac{1}{\alpha^{2}} N(\bar{K})+\frac{2}{\alpha} N(\bar{K})}{1-\frac{1}{\alpha^{2}} N(\bar{K})} \\
& \leq 8 \frac{1-\frac{1}{8 \alpha^{2}} N(\bar{K})+\frac{1}{4 \alpha} N(\bar{K})}{1-\frac{1}{\alpha^{2}} N(\bar{K})} \leq 8 \frac{1}{1-\frac{1}{\alpha^{2}} N(\bar{K})} \frac{1}{1+\frac{1}{8 \alpha^{2}} N(\bar{K})-\frac{1}{4 \alpha} N(\bar{K})} \\
& \leq 8 \frac{1}{1-\left(\frac{1}{4 \alpha}+\frac{7}{8 \alpha^{2}}\right) N(\bar{K})} \leq 8 \frac{1}{1-\frac{1}{2 \alpha} N(\bar{K})},
\end{aligned}
$$

we have

$$
\begin{aligned}
N_{\text {impr }}\left(g_{0}\right) \leq & \frac{2^{5} J}{\alpha^{7}} N(f ; 4 \alpha) \frac{N\left(\bar{K} ; 2 \alpha n^{n+1}\right.}{1-\frac{1}{\alpha} N(\bar{K} ; 2 \alpha)} \\
& \times\left[\frac{8}{\alpha^{2 n-2}} \frac{1}{1-\frac{1}{2 \alpha} N(\bar{K})}+8\left(\frac{8}{\alpha^{2}}\right)^{n-1} \frac{1}{1-\frac{2}{\alpha^{2}} N(\bar{K})} \frac{1}{1-\frac{1}{\alpha^{2}} N(\bar{K})}\right] \\
\leq & \frac{2^{5} J}{\alpha^{7}} N(f ; 4 \alpha) \frac{N\left(\bar{K} ; 2 \alpha n^{n+1}\right.}{1-\frac{1}{\alpha} N(\bar{K} ; 2 \alpha)}\left[\frac{8}{\alpha^{2 n-2}} \frac{1}{1-\frac{1}{2 \alpha} N(\bar{K})}+8\left(\frac{8}{\alpha^{2}}\right)^{n-1} \frac{1}{1-\frac{1}{2 \alpha} N(\bar{K})}\right] \\
\leq & \frac{2^{5} J}{\alpha^{n+6}} N(f ; 4 \alpha) \frac{N(\bar{K} ; 2 \alpha)^{n+1}}{1-\frac{3}{2 \alpha} N(\bar{K} ; 2 \alpha)}[8+8] \\
\leq & \frac{2^{9} J}{\alpha^{n+6}} N(f ; 4 \alpha) \frac{N(\bar{K} ; 2 \alpha)^{n+1}}{1-\frac{3}{2 \alpha} N(\bar{K} ; 2 \alpha)} .
\end{aligned}
$$

By Lemma IX.10 and Remark II.24, there exists a one-legged tail $t_{11}$, a two-legged tail $t_{21}$, each with at least three external legs, and a two-legged tail $\tau_{1}$ with at least four external legs and of degree two in $\eta$ such that

$$
\begin{aligned}
P\left[Q\left(T_{n}(\bar{K})\right)\left(\mathcal{Q}_{K^{\prime}}(: f: \xi, C)\right)\right]= & P\left[Q\left(T_{n+1}(\bar{K})\right)(: f: \xi, C)\right] \\
& +P\left[Q\left(T_{n+1}(\bar{K}), T_{1}(\bar{K})\right)(: f: \xi, C)\right] \\
& +P\left[Q\left(t_{11}+t_{21}+\tau_{1}\right)(: f: \xi, C)\right]+g_{1}(\psi)
\end{aligned}
$$

with

$$
\begin{aligned}
N_{\text {impr }}\left(g_{1}\right) & \leq \frac{2^{9} J}{\alpha^{8}} N(f ; 4 \alpha) N\left(T_{n}(\bar{K}) ; 2 \alpha\right) N(\bar{K} ; 4 \alpha)\left(1+\frac{1}{\alpha^{2}} N(\bar{K} ; 4 \alpha)\right) \\
& \leq \frac{2^{9} J}{\alpha^{2 n+6}} N(f ; 4 \alpha) N(\bar{K} ; 2 \alpha)^{n} \frac{N(\bar{K} ; 4 \alpha)}{1-\frac{1}{\alpha^{2}} N(\bar{K} ; 4 \alpha)} \\
& \leq \frac{2^{9} J}{\alpha^{2 n+6}} N(f ; 4 \alpha) \frac{N(\bar{K} ; 4 \alpha)^{n+1}}{1-\frac{1}{\alpha^{2}} N(\bar{K} ; 4 \alpha)}
\end{aligned}
$$

and

$$
\begin{aligned}
N\left(t_{11}\right), N\left(t_{21}\right) & \leq \frac{2}{\alpha^{2}} N\left(T_{n}(\bar{K})\right) N(\bar{K}) \leq \frac{2}{\alpha^{2 n}} N(\bar{K})^{n+1} \\
N_{\text {eff }}\left(\tau_{1}\right) & \leq \frac{4}{\alpha^{4}} N\left(T_{n}(\bar{K})\right) N(\bar{K})^{2} \leq \frac{4}{\alpha^{2 n+2}} N(\bar{K})^{n+2}
\end{aligned}
$$


Similarly, for $1 \leq \ell \leq n$, by Lemma IX.11, there exists a one-legged tail $t_{12}^{(\ell)}$ and a two-legged tail $t_{22}^{(\ell)}$, each with at least three external legs, and a two-legged tail $\tau^{(\ell)}$ with at least four external legs and of degree two in $\eta$ such that

$$
\begin{aligned}
P\left[Q\left(T_{\ell}(\bar{K}), T_{n}(\bar{K})\right)\left(\mathcal{Q}_{K^{\prime}}(: f: \xi, C)\right)\right]= & P\left[Q\left(T_{\ell+1}(\bar{K}), T_{n+1}(\bar{K})\right)(: f: \xi, C)\right] \\
& +P\left[Q\left(t_{12}^{(\ell)}+t_{22}^{(\ell)}+\tau^{(\ell)}\right)(: f: \xi, C)\right]+g_{2, \ell}(\psi)
\end{aligned}
$$

with

$$
\begin{aligned}
N_{\text {impr }}\left(g_{2, \ell) \leq} \leq\right. & \frac{2^{9} J}{\alpha^{10}} N(f ; 4 \alpha) N\left(T_{n}(\bar{K}) ; 2 \alpha\right) N\left(T_{\ell}(\bar{K}) ; 2 \alpha\right) \\
& \times N(\bar{K} ; 4 \alpha)\left(1+\frac{1}{\alpha^{2}} N(\bar{K} ; 4 \alpha)\right) \\
\leq & \frac{2^{9} J}{\alpha^{2 n+6}} N(f ; 4 \alpha) N(\bar{K} ; 2 \alpha)^{n}\left(\frac{1}{\alpha^{2}} N(\bar{K} ; 2 \alpha)\right)^{\ell} \frac{N(\bar{K} ; 4 \alpha)}{1-\frac{1}{\alpha^{2}} N(\bar{K} ; 4 \alpha)} \\
\leq & \frac{2^{9} J}{\alpha^{2 n+6}} N(f ; 4 \alpha)\left(\frac{1}{\alpha^{2}} N(\bar{K} ; 2 \alpha)\right)^{\ell} \frac{N(\bar{K} ; 4 \alpha)^{n+1}}{1-\frac{1}{\alpha^{2}} N(\bar{K} ; 4 \alpha)}
\end{aligned}
$$

and

$$
\begin{aligned}
N\left(t_{12}^{(\ell)}\right), N\left(t_{22}^{(\ell)}\right) & \leq \frac{12}{\alpha^{4}} N(\bar{K}) N\left(T_{n}(\bar{K})\right) N\left(T_{\ell}(\bar{K})\right) \leq \frac{12}{\alpha^{2(n+\ell)}} N(\bar{K})^{n+\ell+1} \\
N_{\mathrm{eff}}\left(\tau^{(\ell)}\right) & \leq \frac{8}{\alpha^{6}} N(\bar{K})^{2} N\left(T_{n}(\bar{K})\right) N\left(T_{\ell}(\bar{K})\right) \leq \frac{8}{\alpha^{2(n+\ell)+2}} N(\bar{K})^{n+\ell+2} .
\end{aligned}
$$

In particular

$$
\begin{aligned}
N_{\text {impr }}\left(g_{1}\right)+\sum_{\ell=1}^{n} N_{\text {impr }}\left(g_{2, \ell}\right) \leq & \frac{2^{9} J}{\alpha^{2 n+6}} N(f ; 4 \alpha) \frac{N(\bar{K} ; 4 \alpha)^{n+1}}{1-\frac{1}{\alpha^{2}} N(\bar{K} ; 4 \alpha)} \\
& \times \frac{1}{1-\frac{1}{\alpha^{2}} N(\bar{K} ; 2 \alpha)} \\
\leq & \frac{2^{9} J}{\alpha^{2 n+6}} N(f ; 4 \alpha) \frac{N(\bar{K} ; 4 \alpha)^{n+1}}{1-\frac{2}{\alpha^{2}} N(\bar{K} ; 4 \alpha)}, \\
N\left(t_{11}\right)+\sum_{\ell=1}^{n} N\left(t_{12}^{(\ell)}\right), N\left(t_{21}\right)+\sum_{\ell=1}^{n} N\left(t_{22}^{(\ell)}\right) & \leq \frac{12}{\alpha^{2 n}} \frac{N(\bar{K})^{n+1}}{1-\frac{1}{\alpha^{2}} N(\bar{K})}, \\
N_{\text {eff }}\left(\tau_{1}+\sum_{\ell=1}^{n-1} \tau^{(\ell)}+\frac{1}{2} \tau^{(n)}\right) & \leq \frac{8}{\alpha^{2 n+2}} \frac{N(\bar{K})^{n+2}}{1-\frac{1}{\alpha^{2}} N(\bar{K})} .
\end{aligned}
$$

By Lemma IX.12, there exists a two-legged tail $t_{23}$ with at least four external legs such that

$$
P\left[Q\left(t_{1}\right)\left(\mathcal{Q}_{K^{\prime}}(: f: \xi, C)\right)\right]=P\left[Q\left(t_{23}\right)(: f: \xi, C)\right]
$$

and, by the induction hypothesis,

$$
N\left(t_{23}\right) \leq \frac{2}{\alpha^{2}} N\left(t_{1}\right) N(\bar{K}) .
$$

Also, by Lemma IX.10,

$$
P\left[Q\left(t_{2}\right)\left(\mathcal{Q}_{K^{\prime}}(: f: \xi, C)\right)\right]=P\left[Q\left(t_{14}+t_{24}+t_{25}\right)(: f: \xi, C)\right]+g_{3},
$$

where $t_{14}, t_{24}$ are one- resp. two-legged tails with at least three external legs, fulfilling

$$
N\left(t_{14}\right), N\left(t_{24}\right) \leq \frac{2}{\alpha^{2}} N\left(t_{2}\right) N(\bar{K}) .
$$


$t_{25}=t_{2} \circ K^{\prime}$ is a two-legged tail with at least four external legs fulfilling

$$
N\left(t_{25}\right) \leq \frac{1}{\alpha^{2}} N\left(t_{2}\right) N(\bar{K}),
$$

and the $g_{3}$ term obeys

$$
\begin{aligned}
N_{\text {impr }}\left(g_{3}\right) & \leq \frac{2^{9} J}{\alpha^{8}} N(f ; 4 \alpha) N\left(t_{2} ; 2 \alpha\right) N(\bar{K} ; 4 \alpha)\left(1+\frac{1}{\alpha^{2}} N(\bar{K} ; 4 \alpha)\right) \\
& \leq \frac{2^{9} J}{\alpha^{8}} N(f ; 4 \alpha) 4\left(\frac{2}{\alpha^{2}}\right)^{n-1} \frac{N(\bar{K} ; 2 \alpha)^{n}}{1-\frac{1}{2 \alpha^{2}} N(\bar{K} ; 2 \alpha)} \frac{N(\bar{K} ; 4 \alpha)}{1-\frac{1}{\alpha^{2}} N(\bar{K} ; 4 \alpha)} \\
& \leq \frac{2^{n+10} J}{\alpha^{2 n+6}} N(f ; 4 \alpha) \frac{N(\bar{K} ; 4 \alpha)^{+1}}{1-\frac{2}{\alpha^{2}} N(\bar{K} ; 4 \alpha)} .
\end{aligned}
$$

Here we have used that $Q\left(t_{2} \circ K, T_{1}(K)\right)(: f:)$ has at least five external legs so that

$$
P Q\left(t_{2} \circ K^{\prime}, T_{1}(K)\right)(: f:)=0 .
$$

For the same reason, the term " $P Q(\tau)(: f:)$ " of Lemma IX.10 also vanishes.

Finally, by Lemma IX.10,

$$
P\left[Q(\tau)\left(\mathcal{Q}_{K^{\prime}}(: f: \xi, C)\right)\right]=P\left[Q\left(t_{26}\right)(: f: \xi, C)\right],
$$

where $t_{26}=\tau \circ K^{\prime}$ is a two-legged tail with at least four external legs. By Def. IX.8, $N\left(t_{26}\right) \leq \frac{1}{\alpha^{2}} v N(\bar{K}) \quad$ for any effective bound $v$ for $\tau$. Hence, by the induction hypothesis,

$$
N\left(t_{26}\right) \leq \frac{1}{\alpha^{2}} \frac{8}{\alpha^{2 n}} \frac{N(\bar{K})^{n+1}}{1-\frac{1}{\alpha^{2}} N(\bar{K})} N(\bar{K})=\frac{8}{\alpha^{2 n+2}} \frac{N(\bar{K})^{n+2}}{1-\frac{1}{\alpha^{2}} N(\bar{K})} .
$$

Combining the results above, we see that

$$
\begin{aligned}
P \int \operatorname{Ev} \mathcal{R}_{: K: \zeta, D}^{n+1}(: f: \xi, C) d \mu_{D}(\zeta)= & P Q\left(T_{n+1}(\bar{K})\right)(: f: \xi, C) \\
& \left.+\frac{1}{2} \sum_{\substack{\ell, \ell^{\prime} \geq 1 \\
\max \left\{\ell \ell^{\prime}\right\}=n+1}} P Q\left(T_{\ell}(\bar{K}), T_{\ell^{\prime}}(\bar{K})\right)(: f: \xi, C)\right] \\
& +P Q\left(t_{1}^{\prime}+t_{2}^{\prime}+\tau^{\prime}\right)(: f: \xi, C)+h_{n+1}(\psi ; f)
\end{aligned}
$$

with

$$
h_{n+1}(\psi ; f)=h_{n}\left(\psi ; F_{1}(\psi ; f)\right)+g_{0}(\psi)+g_{1}(\psi)+\frac{1}{2} g_{2, n}(\psi)+\sum_{\ell=1}^{n-1} g_{2, \ell}(\psi)+g_{3}
$$

one- resp. two-legged tails with at least three external legs,

$$
\begin{aligned}
& t_{1}^{\prime}=t_{11}+\frac{1}{2} t_{12}^{(n)}+\sum_{\ell=1}^{n-1} t_{12}^{(\ell)}+t_{14}, \\
& t_{2}^{\prime}=t_{21}+\frac{1}{2} t_{22}^{(n)}+\sum_{\ell=1}^{n-1} t_{22}^{(\ell)}+t_{23}+t_{24}+t_{25}+t_{26},
\end{aligned}
$$

and the two-legged tail with four external legs and degree two in $\eta$

$$
\tau^{\prime}=\tau_{1}+\frac{1}{2} \tau^{(n)}+\sum_{\ell=1}^{n-1} \tau^{(\ell)}
$$


By the estimates obtained above

$$
\begin{aligned}
N\left(t_{1}^{\prime}\right), N\left(t_{2}^{\prime}\right) \leq & \frac{12}{\alpha^{2 n}} \frac{N(\bar{K})^{n+1}}{1-\frac{1}{\alpha^{2}} N(\bar{K})}+\frac{1}{\alpha^{2}} N(\bar{K})\left[2 N\left(t_{1}\right)+2 N\left(t_{2}\right)+N\left(t_{2}\right)\right] \\
& +\frac{8}{\alpha^{2 n+2}} \frac{N(\bar{K})^{n+2}}{1-\frac{1}{\alpha^{2}} N(\bar{K})} \\
\leq & \frac{12}{\alpha^{2 n}} \frac{N(\bar{K})^{n+1}}{1-\frac{1}{\alpha^{2}} N(\bar{K})}\left(1+\frac{2}{3 \alpha^{2}} N(\bar{K})\right)+\frac{5}{\alpha^{2}} N(\bar{K}) 4\left(\frac{8}{\alpha^{2}}\right)^{n-1} \frac{N(\bar{K} ; \alpha)^{n}}{1-\frac{2}{\alpha^{2}} N(\bar{K} ; \alpha)} \\
\leq & \frac{12}{\alpha^{2 n}} \frac{N(\bar{K})^{n+1}}{1-\frac{2}{\alpha^{2}} N(\bar{K})}+\frac{5}{2}\left(\frac{8}{\alpha^{2}}\right)^{n} \frac{N(\bar{K} ; \alpha)^{n+1}}{1-\frac{2}{\alpha^{2}} N(\bar{K} ; \alpha)} \\
\leq & \left(\frac{12}{8^{n}}+\frac{5}{2}\right)\left(\frac{8}{\alpha^{2}}\right)^{n} \frac{N(\bar{K} ; \alpha)^{n+1}}{1-\frac{2}{\alpha^{2}} N(\bar{K} ; \alpha)} \\
\leq & 4\left(\frac{8}{\alpha^{2}}\right)^{n} \frac{N(\bar{K} ; \alpha)^{n+1}}{1-\frac{2}{\alpha^{2}} N(\bar{K} ; \alpha)}, \\
N_{\text {eff }}\left(\tau^{\prime}\right) \leq & \frac{8}{\alpha^{2 n+2}} \frac{N(\bar{K})^{n+2}}{1-\frac{1}{\alpha^{2}} N(\bar{K})},
\end{aligned}
$$

and

$$
\begin{aligned}
& N_{\text {impr }}\left(g_{0}\right)+N_{\text {impr }}\left(g_{1}\right)+\sum_{\ell^{\prime}=1}^{n} N_{\text {impr }}\left(g_{2, \ell}\right)+N_{\text {impr }}\left(g_{3}\right) \\
& \quad \leq \frac{2^{9} J}{\alpha^{n+6}} N(f ; 4 \alpha) \frac{N(\bar{K} ; 4 \alpha)^{n+1}}{1-\frac{3}{2 \alpha} N(\bar{K} ; 4 \alpha)}\left[1+\frac{1}{\alpha^{n}}+\frac{2^{n+1}}{\alpha^{n}}\right] \\
& \quad \leq \frac{2^{10} J}{\alpha^{n+6}} N(f ; 4 \alpha) \frac{N(\bar{K} ; 4 \alpha)^{n+1}}{1-\frac{3}{2 \alpha} N(\bar{K} ; 4 \alpha)},
\end{aligned}
$$

so that

$$
\begin{aligned}
N_{\text {impr }}\left(h_{n+1}(\psi ; f)\right) \leq & N_{\text {impr }}\left(h_{n}\left(\psi ; F_{1}(\psi ; f)\right)\right)+N_{\text {impr }}\left(g_{0}\right)+N_{\text {impr }}\left(g_{1}\right) \\
& +\sum_{\ell^{\prime}=1}^{n} N_{\text {impr }}\left(g_{2, \ell}\right)+N_{\text {impr }}\left(g_{3}\right) \\
\leq & \frac{2^{10} J}{\alpha^{5}} \sum_{m=1}^{n} \frac{1}{\alpha^{n+1-m}} \frac{N(\bar{K} ; 4 \alpha)^{n+1-m}}{1-\frac{3}{2 \alpha} N(\bar{K} ; 4 \alpha)} N\left(F_{m}(\psi ; f) ; 4 \alpha\right) \\
& +\frac{2^{10} J}{\alpha^{n+6}} N(f ; 4 \alpha) \frac{N(\bar{K} ; 4 \alpha)^{n+1}}{1-\frac{3}{2 \alpha} N(\bar{K} ; 4 \alpha)} \\
\leq & \frac{2^{10} J}{\alpha^{5}} \sum_{m=0}^{n} \frac{1}{\alpha^{n+1-m}} \frac{N(\bar{K} ; 4 \alpha)^{n+1-m}}{1-\frac{3}{2 \alpha} N(\bar{K} ; 4 \alpha)} N\left(F_{m}(\psi ; f) ; 4 \alpha\right),
\end{aligned}
$$

since $F_{0}(\psi ; f)=f$.

Corollary IX.16. Let $K\left(\psi ; \zeta ; \xi, \xi^{\prime}, \eta\right)$ be a Grassmann function that vanishes for $\eta=0$ and has degree at least four overall. Furthermore let $f(\psi ; \zeta ; \xi)$ be a Grassmann function of degree at least four in the variables $\psi, \zeta, \xi$. Set

$$
\begin{aligned}
h(\psi)= & P\left[\int \operatorname{Ev} \frac{1}{1-\mathcal{R}_{: K: \zeta, D}, C}\left(: f: \xi_{\zeta, D}\right) d \mu_{D}(\zeta)-: f(\psi ; 0 ; \xi): \xi, C\right. \\
& \left.-\sum_{\ell=1}^{\infty} Q\left(T_{\ell}(\bar{K})\right)(: f: \xi, C)-\frac{1}{2} \sum_{\ell, \ell^{\prime} \geq 1} Q\left(T_{\ell}(\bar{K}), T_{\ell^{\prime}}(\bar{K})\right)(: f: \xi, C)\right] .
\end{aligned}
$$

If $\alpha \geq 8$ and $N(K ; 16 \alpha)_{\mathbf{0}}<\frac{1}{3} \alpha$, then

$$
N_{\text {impr }}(h) \leq \frac{2^{10} J}{\alpha^{6}} N(f ; 16 \alpha) \frac{N(K ; 16 \alpha)}{1-\frac{3}{\alpha} N(K ; 16 \alpha)} .
$$


If $K\left(\psi ; \zeta ; \xi, \xi^{\prime}, \eta\right)=\hat{U}\left(\psi+\zeta ; \xi+\xi^{\prime}+\eta\right)-\hat{U}\left(\psi+\zeta ; \xi+\xi^{\prime}\right), \alpha \geq 8$ and $N(\hat{U} ; 32 \alpha)_{\mathbf{0}}$ $<\frac{1}{3} \alpha$, then

$$
N_{\text {impr }}(h) \leq \frac{2^{10} J}{\alpha^{6}} N(f ; 16 \alpha) \frac{N(\hat{U} ; 16 \alpha)}{1-\frac{3}{\alpha} N(\hat{U} ; 32 \alpha)} .
$$

Proof. By Prop. IX.15

$$
\begin{aligned}
& P \int\left[\operatorname{Ev} \frac{1}{1-\mathcal{R}_{: K: \zeta, D}, C}(: f: \underset{\zeta, D}{: \xi, C})-: f: \underset{\zeta, D}{: \xi, C}\right] d \mu_{D}(\zeta) \\
& =\sum_{\ell=1}^{\infty} P\left[\int \operatorname{Ev} \mathcal{R}_{: K:_{\zeta, D}, C}^{\ell}(: f: \underset{\zeta, D}{\xi, C}) d \mu_{D}(\zeta)\right] \\
& =\sum_{\ell=1}^{\infty} P\left[Q\left(T_{\ell}(\bar{K})\right)(: f: \xi, C)\right]+\frac{1}{2} \sum_{\ell, \ell^{\prime} \geq 1} P\left[Q\left(T_{\ell}(\bar{K}), T_{\ell^{\prime}}(\bar{K})\right)(: f: \xi, C)\right] \\
& +\sum_{\ell=1}^{\infty} h_{\ell}(\psi ; f)+P\left[Q\left(\bar{t}_{1}+\bar{t}_{2}\right)(: f: \xi, C)\right]
\end{aligned}
$$

with a one-legged tail $\bar{t}_{1}$ and a two-legged tail $\bar{t}_{2}$, each with at least three external legs. As the degree of $f$ is at least four, $P\left[Q\left(\bar{t}_{1}+\bar{t}_{2}\right)(: f: \xi, C)\right]=0$. Set $h(\psi)=\sum_{\ell=1}^{\infty} h_{\ell}(\psi ; f)$. Then,

$$
N_{\text {impr }}(h) \leq \frac{2^{10} J}{\alpha^{5}} \sum_{\ell=1}^{\infty} \sum_{m=0}^{\ell-1} \frac{1}{\alpha^{\ell-m}} \frac{N(\bar{K} ; 4 \alpha)^{\ell-m}}{1-\frac{3}{2 \alpha} N(\bar{K} ; 4 \alpha)} N\left(F_{m}(\psi ; f) ; 4 \alpha\right) .
$$

Define $\tilde{F}_{m}(\psi ; f)$ by

$$
: \tilde{F}_{m}(\psi ; f): \xi, C=\operatorname{Ev} \mathcal{R}_{: K:_{\zeta, D}, C}^{m}(: f: \underset{\zeta, D}{: \xi, C}) .
$$

Then, by Remark II.24, followed by Lemma VIII.8 and Lemma III.8,

$$
\begin{aligned}
N\left(F_{m}(\psi ; f) ; 4 \alpha\right) & \leq N\left(\tilde{F}_{m}(\psi ; f) ; 8 \alpha\right) \\
& \leq\left(\frac{1}{32 \alpha^{2}} \frac{N\left(: K:_{\zeta, D} ; 8 \alpha\right)}{1-\frac{1}{32 \alpha^{2}} N\left(: K:_{\zeta, D} ; 8 \alpha\right)}\right)^{m} N\left(: f:_{\zeta, D} ; 8 \alpha\right)
\end{aligned}
$$

so that

$$
\begin{aligned}
& N_{\text {impr }}(h) \leq \frac{2^{10} J}{\alpha^{5}} \sum_{\ell=1}^{\infty} \sum_{m=0}^{\ell-1} \frac{1}{\alpha^{\ell-m}} \frac{N(\bar{K} ; 4 \alpha)^{\ell-m}}{1-\frac{3}{2 \alpha} N(\bar{K} ; 4 \alpha)}\left(\frac{\frac{1}{32 \alpha^{2}} N\left(: K:_{\zeta, D} ; 8 \alpha\right)}{1-\frac{1}{32 \alpha^{2}} N\left(: K:_{\zeta, D} ; 8 \alpha\right)}\right)^{m} N(f ; 16 \alpha) \\
& =\frac{2^{10} J}{\alpha^{5}} \sum_{p=1}^{\infty} \sum_{m=0}^{\infty} \frac{1}{\alpha^{p}} \frac{N(\bar{K} ; 4 \alpha)^{p}}{1-\frac{3}{2 \alpha} N(\bar{K} ; 4 \alpha)}\left(\frac{\frac{1}{32 \alpha^{2}} N\left(: K:_{\zeta, D} ; 8 \alpha\right)}{1-\frac{1}{32 \alpha^{2}} N\left(: K:_{\zeta, D} ; 8 \alpha\right)}\right)^{m} N(f ; 16 \alpha)
\end{aligned}
$$

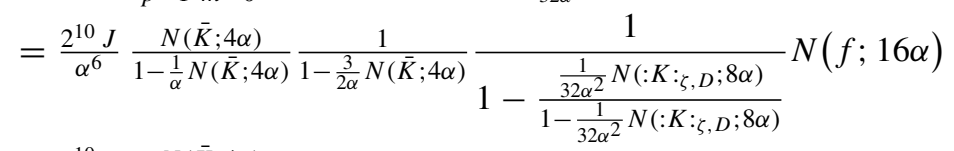

$$
\begin{aligned}
& \leq \frac{2^{10} J}{\alpha^{6}} \frac{N(\bar{K} ; 4 \alpha)}{1-\frac{1}{\alpha} N(\bar{K} ; 4 \alpha)} \frac{1}{1-\frac{3}{2 \alpha} N(\bar{K} ; 4 \alpha)} \frac{1}{1-\frac{1}{16 \alpha^{2}} N\left(: K:_{\zeta, D} ; 8 \alpha\right)} N(f ; 16 \alpha) \\
& \leq \frac{2^{10} J}{\alpha^{6}} \frac{N(K ; 16 \alpha)}{1-\frac{1}{\alpha} N(K ; 16 \alpha)} \frac{1}{1-\frac{3}{2 \alpha} N(K ; 16 \alpha)} \frac{1}{1-\frac{1}{16 \alpha^{2}} N(K ; 16 \alpha)} N(f ; 16 \alpha) \\
& \leq \frac{2^{10} J}{\alpha^{6}} \frac{N(K ; 16 \alpha)}{1-\frac{3}{\alpha} N(K ; 16 \alpha)} N(f ; 16 \alpha) \text {. }
\end{aligned}
$$


If

$$
K\left(\psi ; \zeta ; \xi, \xi^{\prime}, \eta\right)=\hat{U}\left(\psi+\zeta ; \xi+\xi^{\prime}+\eta\right)-\hat{U}\left(\psi+\zeta ; \xi+\xi^{\prime}\right)
$$

so that

$$
\begin{aligned}
\bar{K}\left(\psi ; \zeta, \zeta^{\prime}, \varphi ; \xi, \xi^{\prime}, \eta\right)= & K\left(\psi ; \zeta+\zeta^{\prime}+\varphi ; \xi, \xi^{\prime}, \eta\right) \\
= & \hat{U}\left(\psi+\zeta+\zeta^{\prime}+\varphi ; \xi+\xi^{\prime}+\eta\right) \\
& -\hat{U}\left(\psi+\zeta+\zeta^{\prime}+\varphi ; \xi+\xi^{\prime}\right)
\end{aligned}
$$

and

$: K\left(\psi ; \zeta ; \xi, \xi^{\prime}, \eta\right)_{\zeta, D}=\int\left[\hat{U}\left(\psi+\zeta+\zeta^{\prime} ; \xi+\xi^{\prime}+\eta\right)-\hat{U}\left(\psi+\zeta+\zeta^{\prime} ; \xi+\xi^{\prime}\right)\right] d \mu_{-D}\left(\zeta^{\prime}\right)$

then, by Lemma II.31 and Remark II.24,

$$
\begin{aligned}
N(\bar{K} ; 4 \alpha) & \leq N(\hat{U} ; 16 \alpha), \\
N\left(: K:_{\zeta, D} ; 8 \alpha\right) & \leq N(\hat{U} ; 32 \alpha) .
\end{aligned}
$$

Consequently

$$
\begin{aligned}
N_{\text {impr }}(h) & \leq \frac{2^{10} J}{\alpha^{6}} \frac{N(\bar{K} ; 4 \alpha)}{1-\frac{1}{\alpha} N(\bar{K} ; 4 \alpha)} \frac{1}{1-\frac{3}{2 \alpha} N(\bar{K} ; 4 \alpha)} \frac{1}{1-\frac{1}{16 \alpha^{2}} N(: K: \zeta, D ; 8 \alpha)} N(f ; 16 \alpha) \\
& \leq \frac{2^{10} J}{\alpha^{6}} \frac{N(\hat{U} ; 16 \alpha)}{1-\frac{1}{\alpha} N(\hat{U} ; 32 \alpha)} \frac{1}{1-\frac{3}{2 \alpha} N(\hat{U} ; 32 \alpha)} \frac{1}{1-\frac{1}{16 \alpha^{2}} N(\hat{U} ; 32 \alpha)} N(f ; 16 \alpha) \\
& \leq \frac{2^{10} J}{\alpha^{6}} \frac{N(\hat{U} ; 16 \alpha)}{1-\frac{3}{\alpha} N(\hat{U} ; 32 \alpha)} .
\end{aligned}
$$

$\square$

Proof of Theorem VI.10. Set

$$
K\left(\psi ; \zeta ; \xi, \xi^{\prime}, \eta\right)=\hat{U}\left(\psi+\zeta ; \xi+\xi^{\prime}+\eta\right)-\hat{U}\left(\psi+\zeta ; \xi+\xi^{\prime}\right)
$$

and

$$
\begin{aligned}
\bar{K}\left(\psi ; \zeta, \zeta^{\prime}, \varphi ; \xi, \xi^{\prime}, \eta\right)= & K\left(\psi ; \zeta+\zeta^{\prime}+\varphi ; \xi, \xi^{\prime}, \eta\right) \\
= & \hat{U}\left(\psi+\zeta+\zeta^{\prime}+\varphi ; \xi+\xi^{\prime}+\eta\right) \\
& -\hat{U}\left(\psi+\zeta+\zeta^{\prime}+\varphi ; \xi+\xi^{\prime}\right), \\
\tilde{f}(\psi ; \zeta ; \xi)= & \hat{f}(\psi+\zeta ; \xi) .
\end{aligned}
$$

By Prop. VIII.9

$$
\begin{aligned}
\mathcal{S}_{U, C}(f) & =\mathcal{S}_{U, C}(: \tilde{f}(\psi ; 0 ; \xi): \underset{\psi, C}{\xi, D}) \\
& =: \iint \operatorname{Ev} \frac{1}{1-\mathcal{R}_{: K: \zeta, D}, C}(: \tilde{f}: \underset{\xi, C}{\xi, D}) d \mu_{C}(\xi) d \mu_{D}(\zeta){ }_{\psi, D}
\end{aligned}
$$

so that

$$
P f^{\prime}=P \int \operatorname{Ev} \frac{1}{1-\mathcal{R}_{: K: \zeta, D}, C}(: \underset{\zeta, D}{\tilde{f}:, C}) d \mu_{D}(\zeta)
$$


Therefore, by Cor. IX.16 and Remark II.24, there is $g(\psi)$ with

$$
P f^{\prime}=P\left[\hat{f}(\psi ; 0)+\sum_{\ell=1}^{\infty} Q\left(T_{\ell}(\bar{K})\right)\left(: \tilde{f}_{\xi, C}\right)+\frac{1}{2} \sum_{\ell, \ell^{\prime} \geq 1} Q\left(T_{\ell}(\bar{K}), T_{\ell^{\prime}}(\bar{K})\right)\left(: \tilde{f}_{\xi, C}\right)\right]+g
$$

and

$$
\begin{aligned}
N_{\text {impr }}(g) & \leq \frac{2^{10} J}{\alpha^{6}} \frac{N(\hat{U} ; 32 \alpha)}{1-\frac{3}{\alpha} N(\hat{U} ; 32 \alpha)} N(\tilde{f} ; 16 \alpha) \\
& \leq \frac{2^{10} J}{\alpha^{6}} \frac{N(\hat{U} ; 32 \alpha)}{1-\frac{3}{\alpha} N(\hat{U} ; 32 \alpha)} N(\hat{f} ; 32 \alpha) .
\end{aligned}
$$

Since $\hat{f}$ and $K$ have degree at least four overall,

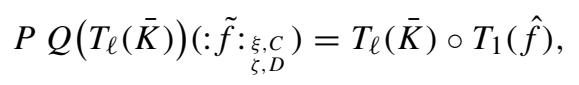

and by Remark VI.9,

$$
P Q\left(T_{\ell}(\bar{K}), T_{\ell^{\prime}}(\bar{K})\right)(: \underset{\zeta, D}{\tilde{f}:, C})=T_{\ell}(\bar{K}) \circ \operatorname{Rung}(\hat{f}) \circ T_{\ell^{\prime}}(\bar{K}) .
$$

Furthermore, by part (ii) of Remark IX.14, $T_{\ell}(\bar{K})=T_{\ell}(\hat{U})$. Thus

$$
P f^{\prime}=P \hat{f}(\psi, 0)+\sum_{\ell=1}^{\infty} T_{\ell}(\hat{U}) \circ T_{1}(\hat{f})+\frac{1}{2} \sum_{\ell, \ell^{\prime} \geq 1} T_{\ell}(\hat{U}) \circ \operatorname{Rung}(\hat{f}) \circ T_{\ell^{\prime}}(\hat{U})+g .
$$

\section{Example: A Vector Model}

We consider a model, which while simple, still captures the main features of one scale of a many fermion model. Let $\mathcal{F}$ be a finite set of at least two "colours" (Farben) and $\mathcal{X}$ be a finite set of points in "space-time". Let $V$ be the complex vector space with basis $\left\{\xi_{c, x} \mid c \in \mathcal{F}, x \in \mathcal{X}\right\}$ and $V^{\prime}$ be the complex vector space with basis $\left\{\psi_{c, x} \mid c \in \mathcal{F}, x \in \mathcal{X}\right\}$. Let $C\left(x, x^{\prime}\right), x, x^{\prime} \in \mathcal{X}$ be a skew symmetric matrix and define the covariance

$$
C\left(\xi_{c, x}, \xi_{c^{\prime}, x^{\prime}}\right)=\delta_{c, c^{\prime}} C\left(x, x^{\prime}\right) .
$$

An antisymmetric function $W$ on $(\mathcal{F} \times \mathcal{X})^{n}$, with $n$ even, is said to be colour preserving if it is of the form

$$
W\left(\left(c_{1}, x_{1}\right), \cdots\left(c_{n}, x_{n}\right)\right)=\operatorname{Ant}\left[\delta_{c_{1}, c_{2}} \cdots \delta_{c_{n-1}, c_{n}} w\left(x_{1}, \cdots, x_{n}\right)\right],
$$

where Ant means antisymmetrization. An example, with $n=2$, is the function $\delta_{c, c^{\prime}} C\left(x, x^{\prime}\right)$. An even element $\mathcal{W}$ of the Grassmann algebra $\wedge V$ is said to be colour preserving if it is of the form

$$
\mathcal{W}=\sum_{n \in 2 \mathbb{N}} \sum_{\substack{c_{1}, \cdots, c_{n} \in \mathcal{F} \\ x_{1}, \cdots, x_{n} \in \mathcal{X}}} W_{n}\left(\left(c_{1}, x_{1}\right), \cdots\left(c_{n}, x_{n}\right)\right) \xi_{c_{1}, x_{1}} \cdots \xi_{c_{n}, x_{n}}
$$

with each coefficient function $W_{n}$ colour preserving. 
For $p$ odd, we define the (0-dimensional) norm $\|\varphi\|_{p}$ of a complex valued function $\varphi$ on $(\mathcal{F} \times \mathcal{X})^{n}$ by

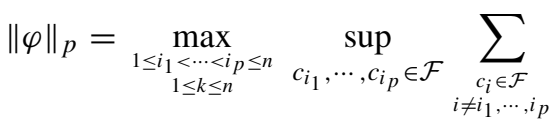

$$
\begin{aligned}
& \times \sup _{x_{k} \in \mathcal{X}} \sum_{\substack{x_{j} \in \mathcal{X} \\
j \neq k}}\left|\varphi\left(\left(c_{1}, x_{1}\right), \cdots\left(c_{n}, x_{n}\right)\right)\right| .
\end{aligned}
$$

Also, for a colour preserving function $W$ on $(\mathcal{F} \times \mathcal{X})^{n}$, we define

$$
\|W\|=\inf \left\{\max _{1 \leq k \leq n} \sup _{x_{k} \in \mathcal{X}} \sum_{\substack{x_{j} \in \mathcal{X} \\ j \neq k}}\left|w\left(x_{1}, \cdots, x_{n}\right)\right| \mid w \text { satisfies (X.1) }\right\} .
$$

Then

$$
\begin{aligned}
\|W\|_{p} & \leq|\mathcal{F}|^{\frac{n-p-1}{2}} \mid\|W\|, \\
\|W\| \| & \leq(n-1) ! !\|W\|_{n-1} \quad \text { if } \quad|\mathcal{F}| \geq \frac{n}{2} .
\end{aligned}
$$

In particular, if $n=4$,

$$
\|W\|_{1} \leq|\mathcal{F}|\left\|W \left|\|\leq 3|\mathcal{F}|\| W \|_{3} .\right.\right.
$$

Every element $f \in V^{\otimes n}$ has a unique representation

$$
f=\sum_{\substack{c_{1}, \cdots, c_{n} \in \mathcal{F} \\ x_{1}, \cdots, x_{n} \in \mathcal{X}}} \varphi\left(\left(c_{1}, x_{1}\right), \cdots\left(c_{n}, x_{n}\right)\right) \xi_{c_{1}, x_{1}} \otimes \cdots \otimes \xi_{c_{n}, x_{n}}
$$

with $\varphi$ a complex valued function on $(\mathcal{F} \times \mathcal{X})^{n}$. We set

$$
\|f\|_{p}=\|\varphi\|_{p} .
$$

Observe that, for each odd $p,\|\cdot\|_{p}$ is a family of symmetric norms on the spaces $V^{\otimes n}$, in the sense of Def. II.18.

Proposition X.1. Suppose that $\mathcal{X}$ is a disjoint union of two subsets $\mathcal{X}_{a}$ and $\mathcal{X}_{c}$ such that

$$
C\left(x, x^{\prime}\right)=0 \quad \text { if both } x, x^{\prime} \in \mathcal{X}_{a} \text { or both } x, x^{\prime} \in \mathcal{X}_{c} .
$$

Assume furthermore that there is a Hilbert space $\mathcal{H}$ and vectors $w_{x} \in \mathcal{H}, x \in \mathcal{X}$ such that

$$
C\left(x, x^{\prime}\right)=\left\langle w_{x}, w_{x^{\prime}}\right\rangle_{\mathcal{H}} \quad \text { for all } x \in \mathcal{X}_{a}, x^{\prime} \in \mathcal{X}_{c} .
$$

Set

$$
\mathrm{b}=2 \sup _{x \in \mathcal{X}}\left\|w_{x}\right\|,
$$

Then $(C, 0)$ has integration constants $\mathfrak{c}, \mathrm{b}$ for the configuration $\|\cdot\|_{p}$ of seminorms, in the sense of Def. VI.13. 
Proof. We first verify that $\mathrm{b}$ is an integral bound for $C$ with respect to each family $\|\cdot\|_{p}$ of seminorms. Set

$$
\begin{gathered}
\mathcal{H}^{\prime}=L^{2}(\mathcal{F}) \otimes \mathcal{H}, \\
w_{c, x}^{\prime}=\delta_{c} \otimes w_{x},
\end{gathered}
$$

where $\delta_{c}$ is the function on $\mathcal{F}$ which vanishes except at $c$ and takes the value one there. Then, for all $c, c^{\prime} \in \mathcal{F}$,

$$
C\left(\xi_{c, x}, \xi_{c^{\prime}, x^{\prime}}\right)=0 \quad \text { if both } x, x^{\prime} \in \mathcal{X}_{a} \text { or both } x, x^{\prime} \in \mathcal{X}_{c}
$$

and

$$
C\left(\xi_{c, x}, \xi_{c^{\prime}, x^{\prime}}\right)=\delta_{c, c^{\prime}}\left\langle w_{x}, w_{x^{\prime}}\right\rangle_{\mathcal{H}}=\left\langle w_{c, x}^{\prime}, w_{c^{\prime}, x^{\prime}}^{\prime}\right\rangle_{\mathcal{H}^{\prime}} \quad \text { for all } x \in \mathcal{X}_{a}, x^{\prime} \in \mathcal{X}_{c} .
$$

Furthermore

$$
\left\|w_{c, x}^{\prime}\right\|_{\mathcal{H}^{\prime}} \leq\left\|w_{x}\right\|_{\mathcal{H}} \leq \frac{\mathrm{b}}{2}
$$

Let $V_{c}$ (respectively $V_{a}$ ) be the subspace of $V$ generated by $\left\{\xi_{c, x} \mid x \in \mathcal{X}_{c}, c \in \mathcal{F}\right\}$ (respectively $\left\{\xi_{c, x} \mid x \in \mathcal{X}_{a}, c \in \mathcal{F}\right\}$ ). By Prop. B.1,

$$
\left|\int \xi_{c_{1}, x_{1}} \cdots \xi_{c_{m}, x_{m}} d \mu_{C}(\xi)\right| \leq\left(\frac{\mathrm{b}}{2}\right)^{m}
$$

As in Example II.26, $\mathrm{b}$ is an integral bound for $C$ with respect to each family $\|\cdot\|_{p}$ of seminorms.

Also

$$
\sup _{\substack{c, c^{\prime} \in \mathcal{F} \\ x, x^{\prime} \in \mathcal{X}}}\left|C\left(\xi_{c, x}, \xi_{c^{\prime}, x^{\prime}}\right)\right|=\sup _{x, x^{\prime} \in \mathcal{X}}\left|C\left(x, x^{\prime}\right)\right| \leq \sup _{x, x^{\prime} \in \mathcal{X}}\left\|w_{x}\right\|\left\|w_{x^{\prime}}\right\| \leq \frac{\mathrm{b}^{2}}{4} .
$$

We now verify the contraction estimates of Def. VI.13. Let

$$
\begin{aligned}
f & =\sum_{\substack{c_{1}, \cdots, c_{n} \in \mathcal{F} \\
x_{1}, \cdots, x_{n} \in \mathcal{X}}} \varphi\left(\left(c_{1}, x_{1}\right), \cdots\left(c_{n}, x_{n}\right)\right) \xi_{c_{1}, x_{1}} \otimes \cdots \otimes \xi_{c_{n}, x_{n}} \in V^{\otimes n}, \\
f^{\prime} & =\sum_{\substack{c_{1}, \cdots, c_{n^{\prime}} \in \mathcal{F} \\
x_{1}, \cdots, x_{n^{\prime}} \in \mathcal{X}}}^{\otimes n} \varphi^{\prime}\left(\left(c_{1}, x_{1}\right), \cdots\left(c_{n^{\prime}}, x_{n^{\prime}}\right)\right) \xi_{c_{1}, x_{1}} \otimes \cdots \otimes \xi_{c_{n^{\prime}}, x_{n^{\prime}}} \in V^{\otimes n^{\prime},},
\end{aligned}
$$

and, for $1 \leq k \leq n$ and $1 \leq k^{\prime} \leq n^{\prime}$,

$$
\begin{aligned}
\Phi_{k}\left(c_{1}, \cdots, c_{n}\right) & =\sup _{x_{k} \in \mathcal{X}} \sum_{\substack{x_{i} \in \mathcal{X} \\
i \neq k}}\left|\varphi\left(\left(c_{1}, x_{1}\right), \cdots\left(c_{n}, x_{n}\right)\right)\right|, \\
\Phi_{k^{\prime}}^{\prime}\left(c_{1}^{\prime}, \cdots, c_{n^{\prime}}^{\prime}\right) & =\sup _{x_{k^{\prime}}^{\prime} \in \mathcal{X}} \sum_{\substack{x_{i}^{\prime} \in \mathcal{X} \\
i \neq k^{\prime}}}\left|\varphi^{\prime}\left(\left(c_{1}^{\prime}, x_{1}^{\prime}\right), \cdots\left(c_{n^{\prime}}^{\prime}, x_{n^{\prime}}^{\prime}\right)\right)\right| .
\end{aligned}
$$

Observe that, for all $1 \leq k \leq n$, all $1 \leq i_{1}<\cdots<i_{p} \leq n$ and all $c_{i_{1}}, \cdots, c_{i_{p}}$,

$$
\sum_{\substack{c_{i} \in \mathcal{F} \\ i \neq i_{1}, \cdots, i_{p}}} \Phi_{k}\left(c_{1}, \cdots, c_{n}\right) \leq\|\varphi\|_{p}=\|f\|_{p}
$$

and similarly for $\Phi^{\prime}$. 
For the first contraction estimate of Def. VI.13, let $1 \leq i \leq n$ and $1 \leq j \leq n^{\prime}$. By the symmetry of the norms, it suffices to consider $i=j=1$. Set

$$
\begin{aligned}
\gamma & \left(\left(c_{2}, x_{2}\right), \cdots\left(c_{n}, x_{n}\right),\left(c_{2}^{\prime}, x_{2}^{\prime}\right), \cdots\left(c_{n^{\prime}}^{\prime}, x_{n^{\prime}}^{\prime}\right)\right) \\
& =\sum_{\substack{c_{1}, c_{1}^{\prime} \in \mathcal{F} \\
x_{1}, x_{1}^{\prime} \in \mathcal{X}}} \varphi\left(\left(c_{1}, x_{1}\right), \cdots,\left(c_{n}, x_{n}\right)\right) \delta_{c_{1}, c_{1}^{\prime}} C\left(x_{1}, x_{1}^{\prime}\right) \varphi^{\prime}\left(\left(c_{1}^{\prime}, x_{1}^{\prime}\right), \cdots,\left(c_{n^{\prime}}^{\prime}, x_{n^{\prime}}^{\prime}\right)\right) .
\end{aligned}
$$

Then

$$
\begin{aligned}
& \underset{1 \rightarrow n+1}{\mathcal{C} \operatorname{on}_{C}\left(f \otimes f^{\prime}\right)} \\
& =\sum_{\substack{c_{1}, \cdots, c_{n+n^{\prime}-2} \in \mathcal{F} \\
x_{1}, \cdots, x_{n+n^{\prime}-2} \in \mathcal{X}}} \gamma\left(\left(c_{1}, x_{1}\right), \cdots,\left(c_{n+n^{\prime}-2}, x_{n+n^{\prime}-2}\right)\right) \xi_{c_{1}, x_{1}} \otimes \cdots \otimes \xi_{c_{n+n^{\prime}-2}, x_{n+n^{\prime}-2}} .
\end{aligned}
$$

In particular $\left\|\underset{i \rightarrow n+j}{\operatorname{Con}_{C}}\left(f \otimes f^{\prime}\right)\right\|_{p}=\|\gamma\|_{p}$.

Fix any $1 \leq i_{1}<\cdots<i_{p} \leq n+n^{\prime}-2$ and colours $c_{i_{1}}, \cdots, c_{i_{p}}$. Set $q=\max \left\{v \mid i_{v} \leq\right.$ $n-1\}$ with the convention that if $\left\{v \mid i_{v} \leq n-1\right\}=\emptyset$, then $q=0$. Set $j_{1}=$ $i_{1}+1, \cdots, j_{q}=i_{q}+1 ; j_{1}^{\prime}=i_{q+1}-n+2, \cdots, j_{p-q}^{\prime}=i_{p}-n+2$ and

$$
\begin{aligned}
& d_{j_{v}}=c_{i_{v}} v=1, \cdots, q, \\
& d_{j_{v}^{\prime}}^{\prime}=c_{i_{q+v}} v=1, \cdots, p-q .
\end{aligned}
$$

Also fix $1 \leq k \leq n+n^{\prime}-2$. First assume that $k \leq n-1$. We have, for all $c_{1}, \cdots, c_{n+n^{\prime}-2}$ $\in \mathcal{F}$,

$$
\begin{aligned}
\sup _{x_{k}} & \sum_{x_{i}, i \neq k}\left|\gamma\left(\left(c_{1}, x_{1}\right), \cdots,\left(c_{n+n^{\prime}-2}, x_{n+n^{\prime}-2}\right)\right)\right| \\
\quad \leq & \sum_{c, c^{\prime} \in \mathcal{F}} \Phi_{k+1}\left(c, c_{1}, \cdots, c_{n-1}\right) \delta_{c, c^{\prime}} \mathrm{c} \Phi_{1}^{\prime}\left(c^{\prime}, c_{n}, \cdots, c_{n+n^{\prime}-2}\right) .
\end{aligned}
$$

Now assume that $q$ is odd. Then $p-q$ is even and

$$
\begin{aligned}
& \sum_{\substack{c_{i} \in \mathcal{F} \\
i \neq i_{1}, \cdots, i_{p}}} \sup _{x_{k}} \sum_{x_{i}, i \neq k}\left|\gamma\left(\left(c_{1}, x_{1}\right), \cdots,\left(c_{n+n^{\prime}-2}, x_{n+n^{\prime}-2}\right)\right)\right| \\
& \leq \mathfrak{c} \sum_{\substack{d_{j} \in \mathcal{F} \\
j \neq 1, j_{1}, \cdots, j_{q}}} \sum_{\substack{d_{\ell}^{\prime} \in \mathcal{F} \\
\ell \neq 1, j_{1}^{\prime}, \cdots, j_{p}^{\prime}-q}} \sum_{c, c^{\prime} \in \mathcal{F}} \Phi_{k+1}\left(c, d_{2}, \cdots, d_{n}\right) \delta_{c, c^{\prime}} \Phi_{1}^{\prime}\left(c^{\prime}, d_{2}^{\prime}, \cdots, d_{n^{\prime}}^{\prime}\right) \\
& \leq \mathfrak{c} \sum_{\substack{d_{j} \in \mathcal{F} \\
j \neq j_{1}, \cdots, j_{q}}} \Phi_{k+1}\left(d_{1}, \cdots, d_{n}\right) \sup _{\substack{d_{1}^{\prime} \\
\ell \neq 1, j_{1}^{\prime}, \cdots, j_{p-q}^{\prime}}} \sum_{\substack{d^{\prime} \in \mathcal{F} \\
f_{1}^{\prime}}} \Phi_{1}^{\prime}\left(d_{1}^{\prime}, d_{2}^{\prime}, \cdots, d_{n^{\prime}}^{\prime}\right) \\
& \leq \mathfrak{c}\|f\|_{q}\left\|f^{\prime}\right\|_{p-q+1} .
\end{aligned}
$$

In the event that $q$ is even, one obtains in a similar way that

$$
\sum_{\substack{c_{i} \in \mathcal{F} \\ i \neq i_{1}, \cdots, i_{p}}} \sup _{x_{k}} \sum_{x_{i}, i \neq k}\left|\gamma\left(\left(c_{1}, x_{1}\right), \cdots,\left(c_{n+n^{\prime}-2}, x_{n+n^{\prime}-2}\right)\right)\right| \leq \mathfrak{c}\|f\|_{q+1}\left\|f^{\prime}\right\|_{p-q} .
$$

The case that $k \geq n$ follows by interchanging the roles of $f$ and $f^{\prime}$. 
For the second contraction estimate of Def. VI.13 it suffices to consider, by the sym-

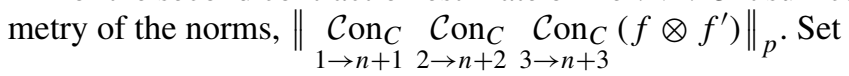

$$
\begin{aligned}
\tilde{\gamma}\left(\left(c_{4}, x_{4}\right), \cdots\left(c_{n}, x_{n}\right),\left(c_{4}^{\prime}, x_{4}^{\prime}\right), \cdots\left(c_{n^{\prime}}^{\prime}, x_{n^{\prime}}^{\prime}\right)\right)= & \sum_{\substack{c_{1}, c_{1}^{\prime}, c_{2}, c_{2}^{\prime}, c_{3}, c_{3}^{\prime} \in \mathcal{F} \\
x_{1}, x_{1}^{\prime}, x_{2}, x_{2}^{\prime}, x_{3}, x_{3}^{\prime} \in \mathcal{X}}} \varphi\left(\left(c_{1}, x_{1}\right), \cdots,\left(c_{n}, x_{n}\right)\right) \delta_{c_{1}, c_{1}^{\prime}} C\left(x_{1}, x_{1}^{\prime}\right) \delta_{c_{2}, c_{2}^{\prime}} \\
& \times C\left(x_{2}, x_{2}^{\prime}\right) \delta_{c_{3}, c_{3}^{\prime}} C\left(x_{3}, x_{3}^{\prime}\right) \varphi^{\prime}\left(\left(c_{1}^{\prime}, x_{1}^{\prime}\right), \cdots,\left(c_{n^{\prime}}^{\prime}, x_{n^{\prime}}^{\prime}\right)\right) .
\end{aligned}
$$

Then

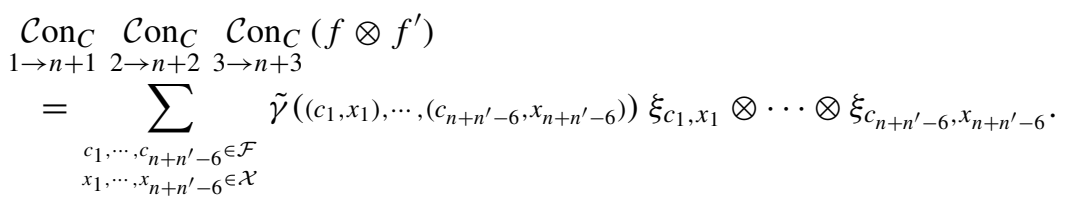

Fix any $1 \leq i_{1}<\cdots<i_{p} \leq n+n^{\prime}-6$ and colours $c_{i_{1}}, \cdots, c_{i_{p}}$. Set $q=\max \left\{v \mid i_{v} \leq\right.$ $n-3\}$. Set $j_{1}=i_{1}+3, \cdots, j_{q}=i_{q}+3 ; j_{1}^{\prime}=i_{q+1}-n+6, \cdots, j_{p-q}^{\prime}=i_{p}-n+6$ and

$$
\begin{aligned}
& d_{j_{v}}=c_{i_{v}}, \quad v=1, \cdots, q \\
& d_{j_{v}^{\prime}}^{\prime \prime}=c_{i_{q+v}}, \quad v=1, \cdots, p-q .
\end{aligned}
$$

Also fix $1 \leq k \leq n+n^{\prime}-6$. First assume that $k \leq n-3$. We have, for all $c_{1}, \cdots, c_{n+n^{\prime}-6} \in$ $\mathcal{F}$,

$$
\begin{aligned}
\sup _{x_{k}} & \sum_{\substack{x_{i}, i \neq k \\
\leq}}\left|\tilde{\gamma}\left(\left(c_{1}, x_{1}\right), \cdots,\left(c_{n+n^{\prime}-6}, x_{n+n^{\prime}-6}\right)\right)\right| \\
\leq & \sum_{\substack{d_{1}, d_{2}, d_{3} \in \mathcal{F} \\
d_{1}^{\prime}, d_{2}^{\prime}, d_{3}^{\prime} \in \mathcal{F}}} \Phi_{k+1}\left(d_{1}, d_{2}, d_{3}, c_{1}, \cdots, c_{n-3}\right) \delta_{d_{1}, d_{1}^{\prime}} \delta_{d_{2}, d_{2}^{\prime}} \delta_{d_{3}, d_{3}^{\prime}} \mathfrak{c}\left[\sup _{y, y^{\prime} \in \mathcal{X}}\left|C\left(y, y^{\prime}\right)\right|\right]^{2} \\
& \times \Phi_{1}^{\prime}\left(d_{1}^{\prime}, d_{2}^{\prime}, d_{3}^{\prime}, c_{n-2}, \cdots, c_{n+n^{\prime}-6}\right) \\
\leq & \mathrm{b}^{4} \mathfrak{c} \sum_{d_{1}, d_{2}, d_{3} \in \mathcal{F}} \Phi_{k+1}\left(d_{1}, d_{2}, d_{3}, c_{1}, \cdots, c_{n-3}\right) \Phi_{1}^{\prime}\left(d_{1}, d_{2}, d_{3}, c_{n-2}, \cdots, c_{n+n^{\prime}-6}\right)
\end{aligned}
$$

by (X.3). Now assume that $q$ is odd. Then

$$
\begin{aligned}
& \sum_{\substack{c_{i} \in \mathcal{F} \\
i \neq i_{1}, \cdots, i_{p}}} \sup _{x_{k}} \sum_{x_{i}, i \neq k}\left|\tilde{\gamma}\left(\left(c_{1}, x_{1}\right), \cdots,\left(c_{n+n^{\prime}-6}, x_{n+n^{\prime}-6}\right)\right)\right| \\
& \leq \mathrm{b}^{4} \mathrm{c} \sum_{\substack{d_{j} \in \mathcal{F} \\
j \neq j_{1}, \cdots, j_{q}}} \Phi_{k+1}\left(d_{1}, \cdots, d_{n}\right) \sup _{\substack{d_{1}^{\prime}, d_{2}^{\prime}, d_{3}^{\prime} \\
\ell \neq 1,2,3, j_{1}^{\prime}, \cdots, j_{p-q}^{\prime}}} \sum_{\substack{d_{\ell}^{\prime} \in \mathcal{F}\\
}} \Phi_{1}^{\prime}\left(d_{1}^{\prime}, d_{2}^{\prime}, \cdots, d_{n^{\prime}}^{\prime}\right) \\
& \leq \mathrm{b}^{4} \mathrm{c}\|f\|_{q}\left\|f^{\prime}\right\|_{p-q+3} .
\end{aligned}
$$

The remaining cases are similar. 
Theorem X.2. Let $W$ be a colour preserving function on $(\mathcal{F} \times \mathcal{X})^{n}$ and

$$
\mathcal{W}=\sum_{\substack{c_{1}, \cdots, c_{4} \in \mathcal{F} \\ x_{1}, \cdots, x_{4} \in \mathcal{X}}} W\left(\left(c_{1}, x_{1}\right), \cdots\left(c_{4}, x_{4}\right)\right) \psi_{c_{1}, x_{1}} \cdots \psi_{c_{4}, x_{4}}
$$

be the associated interaction. Let

$$
\mathcal{W}^{\prime}(\psi)=\Omega_{C}(: \mathcal{W}:)=\log \frac{1}{Z} \int e^{: \mathcal{W}:(\psi+\xi)} d \mu_{C}(\xi) \quad \text { where } \quad Z=\int e^{: \mathcal{W}:(\xi)} d \mu_{C}(\xi)
$$

and $C$ is the covariance of Prop. X.1. Since $C$ and $W$ are colour preserving, we can write

$$
\mathcal{W}^{\prime}=\sum_{n \in 2 \mathbb{N}} \sum_{\substack{c_{1}, \cdots, c_{n} \in \mathcal{F} \\ x_{1}, \cdots, x_{n} \in \mathcal{X}}} W_{n}^{\prime}\left(\left(c_{1}, x_{1}\right), \cdots\left(c_{n}, x_{n}\right)\right) \psi_{c_{1}, x_{1}} \cdots \psi_{c_{n}, x_{n}}
$$

with colour preserving coefficients $W_{n}^{\prime}$. If $\||W| \mid \leq \frac{1}{2^{38} \mathrm{~b}^{2} \mathfrak{c}|\mathcal{F}|}$, then

$$
\sum_{n=6}^{\infty} \alpha_{0}^{n-6} \mathrm{~b}^{n-6}\left\|W_{n}^{\prime}\right\|_{1} \leq 2^{48} \mathfrak{c}|\mathcal{F}|^{2}\|W\|^{2}
$$

with $\alpha_{0}=\frac{1}{\sqrt[3]{2^{29} \mathrm{~b}^{2} \mathfrak{c}|\mathcal{F}|\|W\|}}$, and

$$
\begin{aligned}
\left\|W_{2}^{\prime}\right\| & \leq 2^{61} \mathrm{~b}^{4} \mathrm{c}|\mathcal{F}|\|W\| \|^{2}, \\
\left\|W_{4}^{\prime}-W+\frac{1}{4} \sum_{r=1}^{\infty}(-12)^{r+1} W \circ(\mathcal{C} \circ W)^{r}\right\| & \leq 2^{57} \mathrm{~b}^{2} \mathfrak{c}\|W\|^{2},
\end{aligned}
$$

where $W \circ(\mathcal{C} \circ W)^{r}$ is the ladder

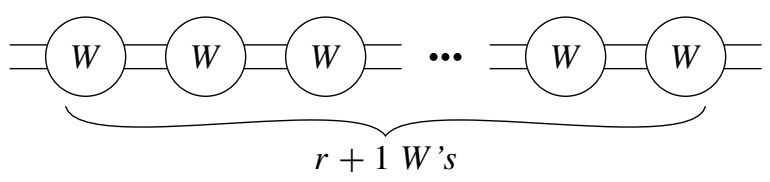

with $r+1$ rungs $W$ and propagator $C$, defined in Appendix $C$.

Proof. Set $J=\frac{1}{|\mathcal{F}|}$. For $f \in V^{\otimes n}$, set

$$
\begin{aligned}
\|f\| & =\|f\|_{1}+|\mathcal{F}|\|f\|_{3}+|\mathcal{F}|^{2}\|f\|_{5}, \\
\|f\|_{\text {impr }} & =\|f\|_{1}+|\mathcal{F}|\|f\|_{3} .
\end{aligned}
$$

By Lemma VI.15, $(C, 0)$ have improved integration constants $\mathfrak{c}, b, J$ for the families $\|\cdot\|$ and $\|\cdot\|_{\text {impr }}$ of seminorms. By (X.2),

$$
\|W\| \leq 2|\mathcal{F}|\|W\| \mid
$$

Let $\alpha_{1}=8$ and $\alpha \in\left\{\alpha_{0}, \alpha_{1}\right\}$. Then $\alpha \geq 8$ and, using the notation of Def. II.23,

$$
N(W ; 64 \alpha)=2^{24} \alpha^{4} \mathrm{~b}^{2} \mathfrak{c}\|W\| \leq 2^{25} \alpha^{4} \mathrm{~b}^{2} \mathfrak{c}|\mathcal{F}|\|W \mid\| \leq \frac{1}{16} \alpha
$$


since $\mathrm{b}^{2} \mathfrak{c}|\mathcal{F}| \| W|| \leq \frac{1}{2^{38}}$. Therefore the hypotheses of Theorem VI.6 are fulfilled. By part (i) of Theorem VI.6,

$$
N\left(\mathcal{W}^{\prime}-\mathcal{W} ; \alpha\right) \leq \frac{1}{2 \alpha^{2}} \frac{N(\mathcal{W} ; 32 \alpha)^{2}}{1-\frac{1}{\alpha^{2}} N(\mathcal{W} ; 32 \alpha)} \leq 2^{48} \alpha^{6} \mathrm{~b}^{4} \mathfrak{c}^{2}|\mathcal{F}|^{2}\|W\|^{2} .
$$

In particular

$$
\frac{\mathfrak{c}}{\mathrm{b}^{2}} \sum_{n=6}^{\infty} \alpha^{n} \mathrm{~b}^{n}\left\|W_{n}^{\prime}\right\|_{1} \leq 2^{48} \alpha^{6} \mathrm{~b}^{4} \mathrm{c}^{2}|\mathcal{F}|^{2}\|W\|^{2}
$$

so that

$$
\sum_{n=6}^{\infty} \alpha^{n-6} \mathrm{~b}^{n-6}\left\|W_{n}^{\prime}\right\|_{1} \leq 2^{48} \mathfrak{c}|\mathcal{F}|^{2}\|W\|^{2}
$$

By part (ii) of Theorem VI.6 and Prop. C.4, both $N_{\text {impr }}\left(W_{2}^{\prime} ; \alpha\right)$ and $N_{\text {impr }}\left(W_{4}^{\prime}-W+\right.$ $\left.\frac{1}{4} \sum_{r=1}^{\infty}(-12)^{r+1} W \circ(\mathcal{C} \circ W)^{r} ; \alpha\right)$ are bounded by

$$
\frac{2^{10} J}{\alpha^{6}} \frac{N(W ; 64 \alpha)^{2}}{1-\frac{8}{\alpha} N(W ; 64 \alpha)} \leq 2^{61} \alpha^{2} \mathrm{~b}^{4} \mathrm{c}^{2}|\mathcal{F}|\|W \mid\|^{2} .
$$

Using (X.2),

$$
\left\|W_{2}^{\prime}\right\|\left|\leq\left\|W_{2}^{\prime}\right\|_{\text {impr }}=\frac{1}{\alpha^{2} \mathfrak{c}} N_{\text {impr }}\left(W_{2}^{\prime} ; \alpha\right) \leq 2{ }^{61} \mathrm{~b}^{4} \mathfrak{c}\right| \mathcal{F} \mid\|W\|^{2}
$$

Similarly, setting $F=W_{4}^{\prime}-W+\frac{1}{4} \sum_{r=1}^{\infty}(-12)^{r+1} W \circ(\mathcal{C} \circ W)^{r}$,

$$
\||F|\| \leq 3\|F\|_{3} \leq \frac{3}{|\mathcal{F}|}\|F\|_{\text {impr }}=\frac{3}{\alpha^{4} \mathrm{~b}^{2} \mathfrak{c}|\mathcal{F}|} N_{\mathrm{impr}}(F ; \alpha) \leq 2^{63} \frac{1}{\alpha^{2}} \mathrm{~b}^{2} \mathrm{c}\|W\|^{2} .
$$

With $\alpha=\alpha_{1}=8$, we get the desired bound.

The $j^{\text {th }}$ shell, $j \geq 1$, of the many fermion model of [FKTf1] behaves qualitatively like the vector model that we have just discussed. The covariance for the $j^{\text {th }}$ shell of that many fermion model is

$$
C^{(j)}(k)=\frac{v^{(j)}(k)}{l k_{0}-e(\mathbf{k})},
$$

where $v^{(j)}(k)$ is approximately the characteristic function with support

$$
S_{j}=\left\{k=\left(k_{0}, \mathbf{k}\right) \in \mathbb{R} \times \mathbb{R}^{d}\left|\frac{1}{M^{j}} \leq\right| \imath k_{0}-e(\mathbf{k}) \mid<\frac{1}{M^{j-1}}\right\} .
$$

To define seminorms in position space that mimic well, in our context, the supremum in momentum space, we introduce sectorizations. We choose a projection $k \mapsto \pi_{F}(k)$ onto the Fermi curve $F$ and a decomposition of the Fermi curve into disjoint intervals $I_{1}, \cdots, I_{N}$ each of length $\mathfrak{l}$ between $\frac{1}{M^{j}}$ and $\frac{1}{M^{j / 2}}$. The sectorization $\Sigma$ is the collection of "sectors" $s_{\ell}=\pi_{F}^{-1}\left(I_{\ell}\right) \cap S_{j}, \ell=1, \cdots, N$. 


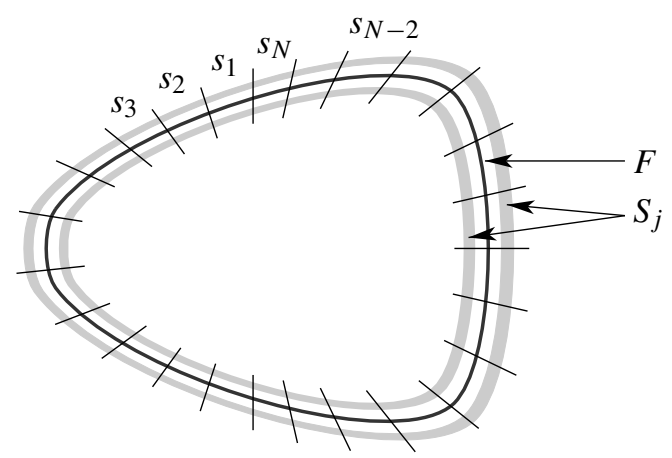

Let $V$ be the complex vector space with basis $\left\{\psi_{s, x}, \bar{\psi}_{s, x} \mid s \in \Sigma, x \in \mathbb{R} \times \mathbb{R}^{2}\right\}$ and define the covariance ${ }^{3}$

$$
C\left(\psi_{s, x}, \psi_{s^{\prime}, x^{\prime}}\right)=C\left(\bar{\psi}_{s, x}, \bar{\psi}_{s^{\prime}, x^{\prime}}\right)=0, \quad C\left(\psi_{s, x}, \bar{\psi}_{s^{\prime}, x^{\prime}}\right)=\delta_{s, s^{\prime}} \int_{s} d^{3} k e^{\imath k \cdot x} C^{(j)}(\mathbf{k}) .
$$

The norms on $V^{\otimes n}$ are similar to those of the vector model. In this case

$$
\mathrm{b}^{2}=\text { const } \frac{\mathfrak{l}}{M^{j}}, \quad \mathfrak{c}=\text { const } M^{j}, \quad J=\frac{1}{|\Sigma|}=\text { const } \mathfrak{l},
$$

and the conclusions of Theorem X.2 become

$$
\begin{aligned}
\left\|W_{2}^{\prime}\right\| \mid & \leq \text { const } \frac{\mathfrak{l}}{M^{j}}\|W\|^{2}, \\
\left\|W_{4}^{\prime}-W+\frac{1}{4} \sum_{r=1}^{\infty}(-12)^{r+1} W \circ(\mathcal{C} \circ W)^{r}\right\| & \leq \text { const } \mathfrak{l}\|W\|^{2}
\end{aligned}
$$

for all four-legged interactions $\mathcal{W}$ whose norm $\|\mid W\|$ is bounded by a sufficiently small, but $j$-independent, constant.

\section{Appendix C. Ladders Expressed in Terms of Kernels}

Let $V$ be a complex vector space and $\left\{\xi_{i}\right\}$ a system of generators for $V$ that is indexed by $i$ in a set $\mathcal{X}$.

Definition C.1. i) Depending on the context, a complex valued function on $\mathcal{X}^{4}$ is called a four legged kernel over $\mathcal{X}$ or a bubble propagator over $\mathcal{X}$.

ii) To a four legged kernel $f$ over $\mathcal{X}$ we associate the Grassmann function

$$
G r(\xi ; f)=\sum_{i_{1}, i_{2}, i_{3}, i_{4} \in \mathcal{X}} f\left(i_{1}, i_{2}, i_{3}, i_{4}\right) \xi_{i_{1}} \xi_{i_{2}} \xi_{i_{3}} \xi_{i_{4}}
$$

in the complex Grassmann algebra $\bigwedge V$.

\footnotetext{
3 We are deliberately ignoring many technical fine points; in particular, we ignore spin, smoothness of partitions of unity, factors of $2 \pi$.
} 
iii) If $A$ and $B$ are covariances on $V$ then the tensor product

$$
A \otimes B\left(i_{1}, i_{2}, i_{3}, i_{4}\right)=A\left(\xi_{i_{1}}, \xi_{i_{3}}\right) B\left(\xi_{i_{2}}, \xi_{i_{4}}\right)
$$

is a bubble propagator over $\mathcal{X}$. In particular we shall consider the bubble propagator

$$
\mathcal{C}=C \otimes C+C \otimes D+D \otimes C,
$$

where $C$ and $D$ are the covariances of Sect. V.2.

iv) Let $f_{1}$ and $f_{2}$ be functions on $\mathcal{X}^{4}$. The operator product of $f_{1}$ and $f_{2}$ is

$$
\left(f_{1} \circ f_{2}\right)\left(i_{1}, i_{2}, i_{3}, i_{4}\right)=\sum_{j_{1}, j_{2} \in \mathcal{X}} f_{1}\left(i_{1}, i_{2}, j_{1}, j_{2}\right) f_{2}\left(j_{1}, j_{2}, i_{3}, i_{4}\right)
$$

whenever the sum is well-defined.

$v)$ Let $f$ be a four legged kernel over $\mathcal{X}$. The antisymmetrization of $f$ is the four legged kernel

$$
(\text { Ant } f)\left(i_{1}, i_{2}, i_{3}, i_{4}\right)=\frac{1}{4 !} \sum_{\pi \in S_{4}} \operatorname{sgn}(\pi) f\left(i_{\pi(1)}, i_{\pi(2)}, i_{\pi(3)}, i_{\pi(4)}\right) .
$$

Clearly $\operatorname{Gr}(\xi ; f)=\operatorname{Gr}(\xi$; Ant $f)$. The kernel $f$ is called antisymmetric if $f=$ Ant $f$.

Lemma C.2. Let

$$
E=\sum_{i_{1}, i_{2}, i_{3}, i_{4} \in \mathcal{X}}\left(e_{202}\left(i_{1}, i_{2}, i_{3}, i_{4}\right) \psi_{i_{1}} \psi_{i_{2}} \xi_{i_{3}}^{\prime} \xi_{i_{4}}^{\prime}+2 e_{211}\left(i_{1}, i_{2}, i_{3}, i_{4}\right) \psi_{i_{1}} \psi_{i_{2}} \zeta_{i_{3}}^{\prime} \xi_{i_{4}}^{\prime}\right)
$$

be an end as in Def. VI.5, where $e_{202}$ and $e_{211}$ are four legged kernels over $\mathcal{X}$ that are antisymmetric under permutations of their four arguments.

i) If

$$
E^{\prime}=\sum_{i_{1}, i_{2}, i_{3}, i_{4} \in \mathcal{X}}\left(e_{202}^{\prime}\left(i_{1}, i_{2}, i_{3}, i_{4}\right) \psi_{i_{1}} \psi_{i_{2}} \xi_{i_{3}}^{\prime} \xi_{i_{4}}^{\prime}+2 e_{211}^{\prime}\left(i_{1}, i_{2}, i_{3}, i_{4}\right) \psi_{i_{1}} \psi_{i_{2}} \zeta_{i_{3}}^{\prime} \xi_{i_{4}}^{\prime}\right)
$$

is another end with antisymmetric four legged kernels $e_{202}^{\prime}$ and $e_{211}^{\prime}$ then

$$
E \circ E^{\prime}=-2 G r\left(\psi ; e_{202} \circ(C \otimes C) \circ e_{202}^{\prime}+e_{211} \circ(C \otimes D+D \otimes C) \circ e_{211}^{\prime}\right) \text {. }
$$

ii) If

$$
\begin{aligned}
\rho= & \sum_{i_{1}, i_{2}, i_{3}, i_{4} \in \mathcal{X}}\left(\rho_{0202}\left(i_{1}, i_{2}, i_{3}, i_{4}\right) \xi_{i_{1}} \xi_{i_{2}} \xi_{i_{3}}^{\prime} \xi_{i_{4}}^{\prime}+2 \rho_{1102}\left(i_{1}, i_{2}, i_{3}, i_{4}\right) \zeta_{i_{1}} \xi_{i_{2}} \xi_{i_{3}}^{\prime} \xi_{i_{4}}^{\prime}\right. \\
& \left.+2 \rho_{0211}\left(i_{1}, i_{2}, i_{3}, i_{4}\right) \xi_{i_{1}} \xi_{i_{2}} \zeta_{i_{3}}^{\prime} \xi_{i_{4}}^{\prime}+4 \rho_{1111}\left(i_{1}, i_{2}, i_{3}, i_{4}\right) \zeta_{i_{1}} \xi_{i_{2}} \zeta_{i_{3}}^{\prime} \xi_{i_{4}}^{\prime}\right)
\end{aligned}
$$

$i s$ a rung with antisymmetric kernels $\rho_{0202}, \rho_{1102}, \rho_{0211}, \rho_{1111}$, then

$E \circ \rho=-2 \sum_{i_{1}, i_{2}, i_{3}, i_{4} \in \mathcal{X}}\left(\epsilon_{202}\left(i_{1}, i_{2}, i_{3}, i_{4}\right) \psi_{i_{1}} \psi_{i_{2}} \xi_{i_{3}}^{\prime} \xi_{i_{4}}^{\prime}+2 \epsilon_{211}\left(i_{1}, i_{2}, i_{3}, i_{4}\right) \psi_{i_{1}} \psi_{i_{2}} \zeta_{i_{3}}^{\prime} \xi_{i_{4}}^{\prime}\right)$

with

$$
\begin{aligned}
& \epsilon_{202}=e_{202} \circ(C \otimes C) \circ \rho_{0202}+e_{211} \circ(C \otimes D+D \otimes C) \circ \rho_{1102}, \\
& \epsilon_{211}=e_{202} \circ(C \otimes C) \circ \rho_{0211}+e_{211} \circ(C \otimes D+D \otimes C) \circ \rho_{1111} .
\end{aligned}
$$


Proof. i)

$$
\begin{aligned}
& E \circ E^{\prime}=\sum_{\substack{i_{1}, i_{2}, i_{3}, i_{4} \\
\ell_{1}, \ell_{2}, \ell_{3}, \ell_{4}}} \iint\left[e_{202}\left(i_{1}, i_{2}, i_{3}, i_{4}\right) \psi_{i_{1}} \psi_{i_{2}}: \xi_{i_{3}} \xi_{i_{4}}: C+2 e_{211}\left(i_{1}, i_{2}, i_{3}, i_{4}\right) \psi_{i_{1}} \psi_{i_{2}} \zeta_{i_{3}} \xi_{i_{4}}\right] \\
& \times\left[e_{202}^{\prime}\left(\ell_{1}, \ell_{2}, \ell_{3}, \ell_{4}\right) \psi_{\ell_{1}} \psi_{\ell_{2}}: \xi_{\ell_{3}} \xi_{\ell_{4}}: C+2 e_{211}^{\prime}\left(\ell_{1}, \ell_{2}, \ell_{3}, \ell_{4}\right) \psi_{\ell_{1}} \psi_{\ell_{2}} \zeta_{\ell_{3}} \xi_{\ell_{4}}\right] \\
& \times d \mu_{C}(\xi) d \mu_{D}(\zeta) \\
& =-\sum_{\substack{i_{1}, i_{2}, i_{3}, i_{4} \\
\ell_{1}, \ell_{2}, \ell_{3}, \ell_{4}}} \psi_{i_{1}} \psi_{i_{2}} \psi_{\ell_{1}} \psi_{\ell_{2}}\left[4 e_{211}\left(i_{1}, i_{2}, i_{3}, i_{4}\right) e_{211}^{\prime}\left(\ell_{1}, \ell_{2}, \ell_{3}, \ell_{4}\right) D\left(\xi_{i_{3}}, \xi_{\ell_{3}}\right) C\left(\xi_{i_{4}}, \xi_{\ell_{4}}\right)\right. \\
& \left.+e_{202}\left(i_{1}, i_{2}, i_{3}, i_{4}\right) e_{202}^{\prime}\left(\ell_{1}, \ell_{2}, \ell_{3}, \ell_{4}\right)\left(C\left(\xi_{i_{3}}, \xi_{\ell_{3}}\right) C\left(\xi_{i_{4}}, \xi_{\ell_{4}}\right)-C\left(\xi_{i_{3}}, \xi_{\ell_{4}}\right) C\left(\xi_{i_{4}}, \xi_{\ell_{3}}\right)\right)\right] \\
& =-\sum_{\substack{i_{1}, i_{2}, j_{1}, j_{2} \\
\ell_{1}, l_{2}, k_{1}, k_{2}}} \psi_{i_{1}} \psi_{i_{2}} \psi_{\ell_{1}} \psi_{\ell_{2}}\left[4 e_{211}\left(i_{1}, i_{2}, j_{1}, j_{2}\right) e_{211}^{\prime}\left(\ell_{1}, \ell_{2}, k_{1}, k_{2}\right) D\left(\zeta_{j_{1}}, \zeta_{k_{1}}\right) C\left(\xi_{j_{2}}, \xi_{k_{2}}\right)\right. \\
& +e_{202}\left(i_{1}, i_{2}, j_{1}, j_{2}\right) e_{202}^{\prime}\left(\ell_{1}, \ell_{2}, k_{1}, k_{2}\right) C\left(\xi_{j_{1}}, \xi_{k_{1}}\right) C\left(\xi_{j_{2}}, \xi_{k_{2}}\right) \\
& \left.-e_{202}\left(i_{1}, i_{2}, j_{1}, j_{2}\right) e_{202}^{\prime}\left(\ell_{1}, \ell_{2}, k_{2}, k_{1}\right) C\left(\xi_{j_{1}}, \xi_{k_{1}}\right) C\left(\xi_{j_{2}}, \xi_{k_{2}}\right)\right] \\
& =-\sum_{i_{1}, i_{2}, \ell_{1}, \ell_{2}} \psi_{i_{1}} \psi_{i_{2}} \psi_{\ell_{1}} \psi_{\ell_{2}}\left[\left(4 e_{211} \circ(C \otimes D) \circ e_{211}^{\prime}\right)\left(i_{1}, i_{2}, \ell_{1}, \ell_{2}\right)\right. \\
& \left.+2\left(e_{202} \circ(C \otimes C) \circ e_{202}^{\prime}\right)\left(i_{1}, i_{2}, \ell_{1}, \ell_{2}\right)\right] \\
& =-2 \sum_{i_{1}, i_{2}, i_{3}, i_{4}} \psi_{i_{1}} \psi_{i_{2}} \psi_{i_{3}} \psi_{i_{4}}\left[\left(e_{211} \circ(C \otimes D+D \otimes C) \circ e_{211}^{\prime}\right)\left(i_{1}, i_{2}, i_{3}, i_{4}\right)\right. \\
& \left.+\left(e_{202} \circ(C \otimes C) \circ e_{202}^{\prime}\right)\left(i_{1}, i_{2}, i_{3}, i_{4}\right)\right] .
\end{aligned}
$$

For the last two equalities we used the antisymmetry of the kernels.

ii) Similarly,

$$
\begin{aligned}
E \circ \rho= & \sum_{\substack{i_{1}, i_{2}, i_{3}, i_{4} \\
\ell_{1}, \ell_{2}, \ell_{3}, \ell_{4}}} \psi_{i_{1}} \psi_{i_{2}} \iint\left[e_{202}\left(i_{1}, i_{2}, i_{3}, i_{4}\right): \xi_{i_{3}} \xi_{i_{4}}: C+2 e_{211}\left(i_{1}, i_{2}, i_{3}, i_{4}\right) \zeta_{i_{3}} \xi_{i_{4}}\right] \\
& \times\left[\left(\rho_{0202}\left(\ell_{1}, \ell_{2}, \ell_{3}, \ell_{4}\right): \xi_{\ell_{1}} \xi_{\ell_{2}}: C+2 \rho_{1102}\left(\ell_{1}, \ell_{2}, \ell_{3}, \ell_{4}\right) \zeta_{\ell_{1}} \xi_{\ell_{2}}\right) \xi_{\ell_{3}}^{\prime} \xi_{\ell_{4}}^{\prime}\right. \\
& \left.+\left(2 \rho_{0211}\left(\ell_{1}, \ell_{2}, \ell_{3}, \ell_{4}\right): \xi_{\ell_{1}} \xi_{\ell_{2}}:_{C}+4 \rho_{1111}\left(\ell_{1}, \ell_{2}, \ell_{3}, \ell_{4}\right) \zeta_{\ell_{1}} \xi_{\ell_{2}}\right) \zeta_{\ell_{3}}^{\prime} \xi_{\ell_{4}}^{\prime}\right] \\
& \times d \mu_{C}(\xi) d \mu_{D}(\zeta) \\
= & -2 \sum_{i_{1}, i_{2}, i_{3}, i_{4}} \psi_{i_{1}} \psi_{i_{2}} \xi_{i_{3}}^{\prime} \xi_{i_{4}}^{\prime}\left[\left(e_{211} \circ(C \otimes D+D \otimes C) \circ \rho_{1102}\right)\left(i_{1}, i_{2}, i_{3}, i_{4}\right)\right. \\
& \left.+\left(e_{202} \circ(C \otimes C) \circ \rho_{0202}\right)\left(i_{1}, i_{2}, i_{3}, i_{4}\right)\right] \\
& -4 \sum \psi_{i_{1}} \psi_{i_{2}} \zeta_{i_{3}}^{\prime} \xi_{i_{4}}^{\prime}\left[\left(e_{211} \circ(C \otimes D+D \otimes C) \circ \rho_{1111}\right)\left(i_{1}, i_{2}, i_{3}, i_{4}\right)\right. \\
& \left.+\left(e_{202} \circ(C \otimes C) \circ \rho_{0211}\right)\left(i_{1}, i_{2}, i_{3}, i_{4}\right)\right] .
\end{aligned}
$$

Lemma C.3. Let $f$ be an antisymmetricfour legged kernel and $n \geq 0$. Set $F=\operatorname{Gr}(f ; \xi)$ and

$$
E_{n}(F)=E(F) \circ \rho(F) \circ \rho(F) \cdots \rho(F)
$$


with $n$ copies of $\rho(F)$, where $E(F)$ and $\rho(F)$ were defined in Def. VI.5.iii. Then

$$
E_{n}(F)=\sum_{i_{1}, i_{2}, i_{3}, i_{4} \in \mathcal{X}} f_{n}\left(i_{1}, i_{2}, i_{3}, i_{4}\right)\left(\psi_{i_{1}} \psi_{i_{2}} \xi_{i_{3}}^{\prime} \xi_{i_{4}}^{\prime}+2 \psi_{i_{1}} \psi_{i_{2}} \zeta_{i_{3}}^{\prime} \xi_{i_{4}}^{\prime}\right)
$$

with

$$
f_{n}=\frac{(-1)^{n}}{2}(12)^{n+1} f \circ(\mathcal{C} \circ f)^{n} .
$$

Proof. Observe that

$$
\begin{aligned}
E(F)= & \left(\begin{array}{l}
4 \\
2
\end{array}\right) \sum_{i_{1}, i_{2}, i_{3}, i_{4}} f\left(i_{1}, i_{2}, i_{3}, i_{4}\right)\left[\psi_{i_{1}} \psi_{i_{2}} \xi_{i_{3}}^{\prime} \xi_{i_{4}}^{\prime}+2 \psi_{i_{1}} \psi_{i_{2}} \zeta_{i_{3}}^{\prime} \xi_{i_{4}}^{\prime}\right], \\
\rho(F)= & \left(\begin{array}{l}
4 \\
2
\end{array}\right) \sum_{i_{1}, i_{2}, i_{3}, i_{4}} f\left(i_{1}, i_{2}, i_{3}, i_{4}\right) \\
& \times\left[\xi_{i_{1}} \xi_{i_{2}} \xi_{i_{3}}^{\prime} \xi_{i_{4}}^{\prime}+2 \zeta_{i_{1}} \xi_{i_{2}} \xi_{i_{3}}^{\prime} \xi_{i_{4}}^{\prime}+2 \xi_{i_{1}} \xi_{i_{2}} \zeta_{i_{3}}^{\prime} \xi_{i_{4}}^{\prime}+4 \zeta_{i_{1}} \xi_{i_{2}} \zeta_{i_{3}} \xi_{i_{4}}^{\prime}\right] .
\end{aligned}
$$

Since $E(F)=E_{0}(F)$, this proves the case $n=0$. We now perform induction on $n$. By Lemma C. 2 and the induction hypothesis

$$
E_{n+1}(F)=\sum_{i_{1}, i_{2}, i_{3}, i_{4} \in \mathcal{X}}\left(\epsilon_{202}\left(i_{1}, i_{2}, i_{3}, i_{4}\right) \psi_{i_{1}} \psi_{i_{2}} \xi_{i_{3}}^{\prime} \xi_{i_{4}}^{\prime}+2 \epsilon_{211}\left(i_{1}, i_{2}, i_{3}, i_{4}\right) \psi_{i_{1}} \psi_{i_{2}} \zeta_{i_{3}}^{\prime} \xi_{i_{4}}^{\prime}\right)
$$

with

$$
\begin{aligned}
\epsilon_{202}=\epsilon_{211} & =-2\left(\begin{array}{l}
4 \\
2
\end{array}\right)\left[f_{n} \circ(C \otimes C) \circ f+f_{n} \circ(C \otimes D+D \otimes C) \circ f\right] \\
& =-12 f_{n} \circ \mathcal{C} \circ f=f_{n+1} .
\end{aligned}
$$

Proposition C.4. Let $f$ be an antisymmetric four legged kernel. Set $F=G r(f ; \xi)$ and let $L_{r}(F)=E(F) \circ \rho(F) \circ \cdots \circ \rho(F) \circ E(F)$ the ladder of length $r \geq 1$ with $r-1$ rungs $\rho(F)$. Then

$$
L_{r}(F)=\frac{(-1)^{r}}{2}(12)^{r+1} G r\left(\psi ; f \circ(\mathcal{C} \circ f)^{r}\right) .
$$

Proof. By Lemma C.2, Lemma C.3 and (C.1),

$$
\begin{aligned}
L_{r}(F) & =E_{r-1}(F) \circ E(F) \\
& =-2\left(\begin{array}{l}
4 \\
2
\end{array}\right) G r\left(\psi ; f_{r-1} \circ(C \otimes C) \circ f+f_{r-1} \circ(C \otimes D+D \otimes C) \circ f\right) \\
& =-12 G r\left(\psi ; f_{r-1} \circ \mathcal{C} \circ f\right) \\
& =\frac{(-1)^{r}}{2}(12)^{r+1} \operatorname{Gr}\left(\psi ; f \circ(\mathcal{C} \circ f)^{r}\right) .
\end{aligned}
$$




\section{Notation}

\begin{tabular}{|c|c|c|}
\hline Not'n & Description & Reference \\
\hline$Z(f)$ & degree zero component of $f$ & Definition II.1.iii \\
\hline$\bigwedge V$ & Grassmann algebra over $V$ & Example II.2 \\
\hline$\bigwedge_{A}^{\prime} V$ & Grassmann algebra over $V$ with coefficients in $A$ & Example II.2 \\
\hline$A_{m}\left[n_{1}, \cdots, n_{r}\right]$ & partially antisymmetric elements of $A_{m} \otimes V^{\otimes\left(n_{1}+\cdots+n_{r}\right)}$ & Definition II.21 \\
\hline$/ \bigwedge E, / \bigwedge_{A} E$ & enlarged algebra & Definition VIII.5 \\
\hline Ev & evaluation map & Definition VIII.5 \\
\hline $\int e^{\Sigma \xi_{i} \zeta_{i}} d \mu_{C}(\xi)$ & $=e^{-1 / 2 \Sigma \zeta_{i} C_{i j} \zeta_{j}}$ Grassmann Gaussian integral & before Definition II.3 \\
\hline$\Omega_{C}(W)(\psi)$ & $=\log \frac{1}{Z} \int e^{W(\psi+\xi)} d \mu_{C}(\xi)$ renormalization group map & Definitions II.3, II.27 \\
\hline $\mathcal{S}(f)$ & $=\frac{1}{Z(U, C)} \int f(\xi) e^{U(\xi)} d \mu_{C}(\xi)$ Schwinger functional & before Remark III.1 \\
\hline$R$ & $R$-operator & before Theorem III.2 \\
\hline$R_{C}\left(K_{1}, \cdots, K_{\ell}\right)$ & $\ell^{\text {th }}$ Taylor coefficient of $R$ & (III.2) - n \\
\hline $\mathcal{R}_{K, C}(f)$ & $\iint e^{: K\left(\xi, \xi^{\prime}, \eta\right): \xi^{\prime}}-1:_{\eta} f(\eta) d \mu_{C}\left(\xi^{\prime}\right) d \mu_{C}(\eta) \cdot{ }_{\xi}$ & Definition III.4 \\
\hline$\tilde{R}_{C}\left(K_{2}, \cdots, K_{\ell}\right)(f)$ & $\iint:\left(\prod_{i=2}^{\ell}: K_{i}\left(\xi, \xi^{\prime}+\xi^{\prime \prime}, \eta^{\prime}\right):_{\xi^{\prime \prime}}\right):_{\eta^{\prime}} f\left(\eta+\eta^{\prime}\right) d \mu_{C}\left(\xi^{\prime \prime}\right) d \mu_{C}\left(\eta^{\prime}\right)$ & (VII.1) \\
\hline$: e^{\Sigma \xi_{i} \zeta_{i}}: \xi, C$ & $=e^{1 / 2 \Sigma \zeta_{i} C_{i j} \zeta_{j}} e^{\Sigma \xi_{i} \zeta_{i}}$ Wick ordering & after Remark II.4 \\
\hline$\underset{i \rightarrow j}{\operatorname{Con}_{C},} \underset{\xi \rightarrow \xi^{\prime}}{\operatorname{Con}_{C}}$ & contractions & Definitions II.5, II.9 \\
\hline $\mathcal{N}_{d}^{3}$ & norm domain & Definition II.14 \\
\hline $\mathfrak{c}$ & contraction bound & Definition II.25.i \\
\hline $\mathrm{b}$ & integral bound & Definition II.25.ii \\
\hline $\mathfrak{c}, \mathrm{b}, J$ & improved integration constants & Definition VI.1 \\
\hline$N(f ; \alpha)$ & $\frac{1}{\mathrm{~b}^{2}} \mathfrak{c} \sum_{m, n_{1}, \cdots, n_{r} \geq 0} \alpha^{|n|} \mathrm{b}^{|n|}\left\|f_{m ; n_{1}, \cdots, n_{r}}\right\|$ & Definition II.23 \\
\hline$\rho(F)$ & rung & Definition VI.5 \\
\hline$E(F)$ & end & Definition VI.5 \\
\hline$L_{r}(F)$ & ladder of length $r$ & Definition VI.5 \\
\hline$\|\cdot\|_{p}$ & configuration of seminorms & Definition VI.13 \\
\hline$P$ & projects : $f(\psi ; \xi): \xi$ to $f_{4,0}(\psi, 0)+f_{2,0}(\psi, 0)$ & Definition VII.8 \\
\hline$Q\left(\bar{K}_{1}, \cdots, \bar{K}_{\ell}\right)$ & enlarged algebra analog of $R_{C}\left(K_{1}, \cdots, K_{\ell}\right)$ & Definition VIII.1 \\
\hline $\operatorname{Gr}(\xi ; f)$ & Grassmann function & Definition C.1.ii \\
\hline$\circ$ & convolution & Definition C.1.iv \\
\hline
\end{tabular}

\section{References}

[BS] Berezin, F., Shubin, M.: The Schrödinger Equation. Dordrecht: Kluwer, 1991. Supplement 3: D.Leites, Quantization and supermanifolds

[FKLT1] Feldman, J., Knörrer, H., Lehmann, D., Trubowitz, E.: Fermi Liquids in Two-Space Dimensions. In: Constructive Physics V. Rivasseau, (ed.), Springer Lecture Notes in Physics 446, Berlin-Heidelberg-New York: Springer, 1995, pp. 267-300

[FKLT2] Feldman, J., Knörrer, H., Lehmann, D., Trubowitz, E.: Are There Two Dimensional Fermi Liquids? In: Proceedings of the XIth International Congress of Mathematical Physics, D. Iagolnitzer, (ed.), Cambridge, MA: International Press, 1995, pp. 440-444

[FKT1] Feldman, J., Knörrer, H., Trubowitz, E.: A Representation for Fermionic Correlation Functions. Commun. Math. Phys. 195, 465-493 (1998)

[FKTf1] Feldman, J., Knörrer, H., Trubowitz, E.: A Two Dimensional Fermi Liquid, Part 1: Overview. Commun. Math. Phys. 247, 1-47 (2004)

[FKTo3] Feldman, J., Knörrer, H., Trubowitz, E.: Single Scale Analysis of Many Fermion Systems, Part 3: Sectorized Norms. Rev. Math. Phys. 15, 1039-1120 (2003)

[FMRT] Feldman, J., Magnen, J., Rivasseau, V., Trubowitz, E.: An Infinite Volume Expansion for Many Fermion Green's Functions. Helv. Phys. Acta 65, 679-721 (1992)

[FST1] Feldman, J., Salmhofer, M., Trubowitz, E.: Perturbation Theory around Non-nested Fermi Surfaces, I: Keeping the Fermi Surface Fixed. J. Stat. Phys. 84, 1209-1336 (1996)

[FST2] Feldman, J., Salmhofer, M., Trubowitz, E.: Regularity of the Moving Fermi Surface: RPA Contributions. Commun. Pure and Appl. Math. LI, 1133-1246 (1998) 
[FST3] Feldman, J., Salmhofer, M., Trubowitz, E.: Regularity of Interacting Nonspherical Fermi Surfaces: The Full Self-Energy. Commun. Pure and Appl. Math. LII, 273-324 (1999)

[FST4] Feldman, J., Salmhofer, M., Trubowitz, E.: An inversion theorem in Fermi surface theory. Commun. Pure and Appl. Math. LIII, 1350-1384 (2000)

Communicated by J.Z. Imbrie 